Portland State University

PDXScholar

Fall 1-9-2018

\title{
Mechanisms and Identification of Unsteady Separation Development and Remediation
}

Matthew Scott Melius

Portland State University

Follow this and additional works at: https://pdxscholar.library.pdx.edu/open_access_etds

Part of the Aerodynamics and Fluid Mechanics Commons, and the Fluid Dynamics Commons Let us know how access to this document benefits you.

\section{Recommended Citation}

Melius, Matthew Scott, "Mechanisms and Identification of Unsteady Separation Development and Remediation" (2018). Dissertations and Theses. Paper 4064.

https://doi.org/10.15760/etd.5948

This Dissertation is brought to you for free and open access. It has been accepted for inclusion in Dissertations and Theses by an authorized administrator of PDXScholar. Please contact us if we can make this document more accessible: pdxscholar@pdx.edu. 
Mechanisms and Identification of

Unsteady Separation Development and Remediation

by

Matthew Scott Melius

A dissertation submitted in partial fulfillment of the requirements for the degree of

Doctor of Philosophy

in

Mechanical Engineering

Dissertation Committee:

Raúl Bayoán Cal, Chair

Karen Mulleners

Gerald Recktenwald

Stefan Talke

Portland State University

2018 


\begin{abstract}
Unsteady flow separation represents a highly complex and important area of study within fluid mechanics. The extent of separation and specific time scales over which it occurs are not fully understood and has significant consequences in numerous industrial applications such as helicopters, jet engines, hydroelectric turbines and wind turbines. A direct consequence of unsteady separation is the erratic movement of the separation point which causes highly dynamic and unpredictable loads on an airfoil. Current computational models underestimate the aerodynamic loads due to the inaccurate prediction of the emergence and severity of unsteady flow separation especially in response to a sudden change in the effective angle of attack. To capture the complex flow phenomena over wind turbine blades during stall development, a scaled three-dimensional non-rotating blade model is designed to be dynamically similar to a rotating full-scale NREL 5MW wind turbine blade. A time-resolved particle image velocimetry (PIV) investigation of flow behavior during the stall cycle examines the processes of stall development and flow reattachment. The flow fields are examined through the application of Eulerian techniques such as proper orthogonal decomposition and empirical mode decomposition to capture unsteady separation characteristics within the flow field. Then, for a higher order description, coherent structures such as vortices and material lines are resolved to fully characterize the flow during a full pitching cycle in a Lagrangian framework. The Eulerian and Lagrangian methods described in the present analysis is extended to investigate the spanwise characteristics within the root section of a three dimensional airfoil.
\end{abstract}


Furthermore, statistical information of the separation point is pursued along four spanwise positions during two cases of unsteady separation. The results of the study describe a critical role of surface vorticity accumulation in unsteady separation and reattachment. Evaluation of the unsteady characteristics of the shear layer reveal evidence that the build-up and shedding of surface vorticity directly influence the dynamic changes in separation point. The quantitative characterization of surface vorticity and shear layer stability enables improved aerodynamic design, but also has a broader implications on the larger discipline of unsteady fluid dynamics. 


\section{Acknowledgements}

I would like to thank all of the individuals that have supported me during this pursuit. First, I must thank my friend and mentor Raúl Bayoán Cal for giving me the opportunity to pursue my curiosities and support throughout the journey. Thank you to Karen Mulleners for being patient and thoughtful while supplying guidance throughout the course of this work. Thanks to Nicholas Hamilton and Drew Wollman for all of the support and inspiration. Thank you to Dr. Recktenwald and Dr. Talke for acting as advisors during the final stages of this dissertation. I would like to also thank my friends and family who have graciously accepted my commitment to further education and met it with enthusiasm.

Lastly I would like to thank my lovely wife Bonnie for all of her continued love and support.

Sincerely,

Matt 


\section{Table of Contents}

Abstract

Acknowledgements iii

List of Figures vi vi vi vis

1 Introduction 1

1.1 Unsteady separation applied to wind energy . . . . . . . . . . . 10

2 Theory 14

2.1 Coherent Structure Identification Techniques . . . . . . . . . . . . . 14

2.2 Lagrangian Coherent Structures . . . . . . . . . . . . . . . 22

3 Experimental Setup $\quad 25$

4 Proper orthogonal decomposition of unsteady separation 31

4.1 Time resolved stall behavior - Vortex identification . . . . . . . . 33

4.2 POD based time scales . . . . . . . . . . . . . . . . 47

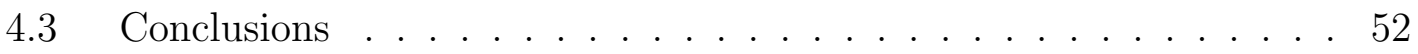


5 Coherent structure interaction during unsteady separation $\quad 56$

5.1 Lagrangian Coherent Structures (LCS) . . . . . . . . . . . 60

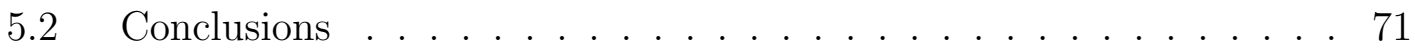

6 The critical role of surface vorticity during unsteady separation $\quad 76$

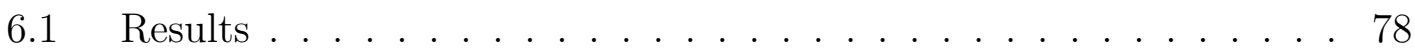

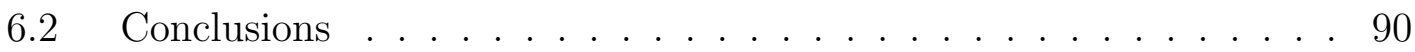

7 Spanwise and pitching rate dependence of peak vorticity and sep$\begin{array}{ll}\text { aration characteristics } & 94\end{array}$

7.1 Peak vorticity statistics . . . . . . . . . . . . . . 94

7.2 Peak vorticity span and speed comparison . . . . . . . . . 105

7.3 Comparison of spanwise and pitch rate dependence of POD coefficients107

7.4 Separation point quantities comparison . . . . . . . . . . 111

7.5 Amplitude ratios ...................... 114

7.6 Amplitude variation with separation point . . . . . . . . . . 117

7.7 External data set comparison . . . . . . . . . . . . . . . 119

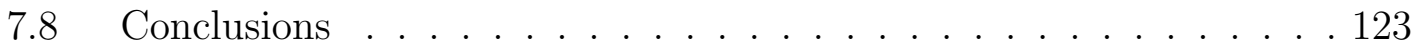

8 Conclusions $\quad 126$

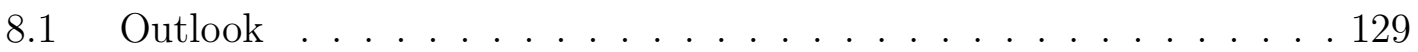

$\begin{array}{ll}\text { Bibliography } & 131\end{array}$ 


\section{List of Figures}

1.1 Examples of unsteady separation captured by (a) computational fluid dynamics and (b) Schlieren photography. . . . . . . . . . . . . 4

1.2 Schematic of theoretical unsteady separation over an airfoil. For consistency of presentation, coordinates are rotated from $(x, y)$ to $(\chi, \phi)$ adjust for the angle of attack, $\alpha$, realative to the freestream velocity, $U_{\infty}$. The perturbed shear layer is plotted as a black dashed line emanating from the separation point (grey 'o') with perturbations caused by departing surface vortices (grey ' $\rightarrow$ ') plotted over a black dashed line which represents a steady shear layer without

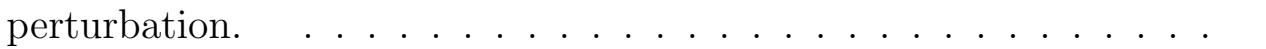

3.1 XFOIL based (a) peak and $(b) x / c=0.25$ pressure distributions, $P_{d}$, for an operational (grey) NREL 5MW wind turbine under steady wind speeds of $U_{w}=15 \mathrm{~m} / \mathrm{s}$ plotted next to the estimated peak pressure for the experimental (orange) model blade under steady free-stream speeds of $U_{\infty}=40 \mathrm{~m} / \mathrm{s}$, (c) experimental design (orange) vs. operational (grey) angles of attack plotted as a function of normalized radial position, $\alpha(r / R)$. . . . . . . . 27 
3.2 Experimental setup: Model turbine blade stands vertically in the $2 \times 1.8 \mathrm{~m}^{2}$ test section, free stream velocity $U_{\infty}=40 \mathrm{~m} / \mathrm{s}$, measurement plane height set to $0.37 m \leq h_{p} \leq 0.58 m, d r / d R=0.065$ between adjacent planes, camera and pitching motor located directly above and below the rotor blade, respectively. . . . . . . .

4.1 PIV snapshot including a) velocity field (vector map) and b) corresponding vorticity field, contours of $\lambda_{2}=0$ (gray), and vortex location and sense of rotation (clockwise/negative in orange and counterclockwise/positive in blue). . . . . . . . . . . . . . . 32

4.2 Angle of attack $(\alpha)$ vs. time $(\tau) \ldots \ldots . \ldots . \ldots . \ldots 34$

4.3 Angle of attack, $\alpha(\tau)$ from $12^{\circ}-24^{\circ}-12^{\circ}$ (gray) plotted with vortex separation angle $\beta$ (blue), and vortex size $\bar{d}_{v}$ (green) as functions of time $(\tau)$ during a dynamic pitching cycle . . . . . . . . . . . 35

4.4 Normalized velocity, $\tilde{u}_{i} / U_{\infty}$, contours and vector fields of instantaneous PIV snapshots, plotted with normalized $x / c$ and $y / c$ coordinates, over a dynamically pitching DU $99-W-350$ crosssection from $\alpha(\tau)=12^{\circ}-24^{\circ}-12^{\circ}$. Vortex areas defined by $\lambda_{2}=0$ contours (gray) surround vortex cores as identified by $\Gamma_{2}-$ criterion which give rotational sense (clockwise in orange and counter-clockwise in blue). . . . . . . . . . . . . . . 36

4.5 Instantaneous Velocity at low angles of attack where trailing edge stall develops briefly before flow reattaches after motion begins. . . 37

4.6 Averaged continuity based calculation of $\partial w / \partial z$ for a pitching cycle of $12^{\circ}-24^{\circ}-12^{\circ}$, plotted with instantaneous velocity vectors. . . . 39 
4.7 Contour plots of the first four spatial POD modes $\psi_{1}, \psi_{2}, \psi_{3}$ and $\psi_{4}$ associated with the experimental motion profile $\alpha(\tau)=12^{\circ} \rightarrow$

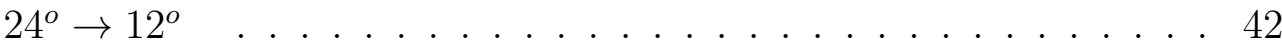

4.8 Normalized POD time coefficients, $a_{1}, a_{2}, a_{3}, a_{4}$ and separation point $f(x / c)$ plotted against angle of attack as a function of time, $\alpha(\tau) \ldots \ldots \ldots \ldots$. . . . . . . . . . . . . . 4

4.9 Reconstruction of the velocity field using the first four POD modes plotted adjacent to the corresponding velocity fields during attached flow, stall development, and fully stalled stages. . . . . . . . 50

4.10 Reconstruction with 2, 3, and 4 modes (columns) of the velocity field using the first four POD modes plotted with the corresponding velocity fields during attached flow, stall development, fully stalled, and reattachment stages (rows). . . . . . . . . . . 53

5.1 Instantaneous velocity field at three angles of attack and times that demonstrate the dynamic nature of stall development. . . . . . . 59

5.2 Instantaneous velocity field over six consecutive PIV snapshots during stall development plotted with attracting (black) and repelling LCS (red), separation point $(\triangle)$, and locations of clockwise (orange) and counter-clockwise (blue) rotating vortex core locations.

5.3 Instantaneous velocity fields over six consecutive PIV snapshots during full stall, plotted with attracting (black) and repelling LCS (red), separation point $(\Delta)$, and locations of clockwise (orange) and counter-clockwise (blue) rotating vortex core locations. . . . . . . 65 
5.4 Instantaneous velocity fields over six consecutive PIV snapshots during reattachment, plotted with attracting (black) and repelling LCS (red), separation point $(\Delta)$, and locations of clockwise (orange) and counter-clockwise (blue) rotating vortex core locations.

5.5 First empirical mode for separation (a), full stall (b), and reattachment (c). . . . . . . . . . . . . . . . . . 68

5.6 Second empirical mode for separation (a), full stall (b), and reattachment (c). . . . . . . . . . . . . . . . 69

5.7 Third empirical mode for separation (a), full stall (b), and reattachment $(\mathrm{c}) . \ldots \ldots \ldots 70 \ldots \ldots$

5.8 Fourth empirical mode for separation, full stall, and reattachment. 71

$6.1 \quad$ (a) Velocity ratio $U_{S L}=0.48 U_{\infty}$ (red) plotted with the shear layer attracting LCS (black) on top of the instantaneous vorticity field during full stall at an angle of $\alpha=24$ (b) Schematic of theoretical unsteady separation over an airfoil. For consistency of presentation, coordinates are rotated to chord based coordinates $(x, y)$, adjusted for the angle of attack, $\alpha$, relative to the freestream velocity, $U_{\infty}$. The perturbed shear layer is plotted as a black line emanating from the separation point (grey 'o') with perturbations caused by departing surface vortices (grey ' $\rightarrow$ ') plotted over a black dashed line which represents a steady shear layer without perturbation. . . 80 
6.2 (a) Example of shear layer stability during reattachment. Three sequential shear layers plotted as $\tau$ (dark red), $\tau_{1}$ (red), and $\left(\tau_{2}\right.$ (light red) along a mean shear layer (black dashed) based on the shear layers of $\pm 5 \tau$ with $A$ as the perturbation amplitude. On the lower axis, normalized vorticity maxima, $\left|\omega^{*}\right|$, taken from along the shear layer, plotted as function of chordwise position $(x / c) . \quad$. . . 81

6.3 Peak vorticity at the point of separation (gold) plotted as a function of $\tau$, plotted along side the separation point (green) and the motion profile (grey), with the mean $\left|\omega^{*}\right|$, standard deviation, skewness, $S$, and kurtosis, $K$, plotted as functions of time for the selected stall stage at $z / R=0.317$ at a pitch rate of $\dot{\alpha}=17.14 \ldots$. . . . . . . 82

6.4 Shear layer tortuosity (green) plotted with separation point (black) and pitching motion(grey). . . . . . . . . . . . 86

6.5 Maximum perturbation amplitude vs separation point and pitching motion. .................... . . 87

6.6 Separation point perturbation amplitude vs separation point and pitching motion. . . . . . . . . . . . . . . 89

7.1 Peak vorticity at the point of separation (gold) plotted as a function of $\tau$, plotted along side the separation point (green) and the motion profile (grey), with the mean, standard deviation, skewness, $S$, and kurtosis, $K$, plotted as functions of time for the selected stall stage at $z / R=0.187$ at a pitch rate of $\dot{\alpha}=17.14 \ldots . . . . . . .995$ 
7.2 Peak vorticity at the point of separation (gold) plotted as a function of $\tau$, plotted along side the separation point (green) and the motion profile (grey), with the mean, standard deviation, skewness, $S$, and kurtosis, $K$, plotted as functions of time for the selected stall stage at $z / R=0.187$ at a pitch rate of $\dot{\alpha}=34.28$. . . . . . . . . . . 97

7.3 Peak vorticity at the point of separation (gold) plotted as a function of $\tau$, plotted along side the separation point (green) and the motion profile (grey), with the mean, standard deviation, skewness, $S$, and kurtosis, $K$, plotted as functions of time for the selected stall stage at $z / R=0.252$ at a pitch rate of $\dot{\alpha}=17.14$. . . . . . . . . . . 98

7.4 Peak vorticity at the point of separation (gold) plotted as a function of $\tau$, plotted along side the separation point (green) and the motion profile (grey), with the mean, standard deviation, skewness, $S$, and kurtosis, $K$, plotted as functions of time for the selected stall stage at $r / R=0.252$ at a pitch rate of $\dot{\alpha}=34.28$. . . . . . . . . . . . . 99

7.5 Peak vorticity at the point of separation (gold) plotted as a function of $\tau$, plotted along side the separation point (green) and the motion profile (grey), with the mean, standard deviation, skewness, $S$, and kurtosis, $K$, plotted as functions of time for the selected stall stage at $r / R=0.317$ at a pitch rate of $\dot{\alpha}=17.14$. . . . . . . . . . . 101 
7.6 Peak vorticity at the point of separation (gold) plotted as a function of $\tau$, plotted along side the separation point (green) and the motion profile (grey), with the mean, standard deviation, skewness, $S$, and kurtosis, $K$, plotted as functions of time for the selected stall stage at $r / R=0.382$ at a pitch rate of $\dot{\alpha}=17.14$. . . . . . . . . . . . . 102

7.7 Peak vorticity at the point of separation (gold) plotted as a function of $\tau$, plotted along side the separation point (green) and the motion profile (grey), with the mean, standard deviation, skewness, $S$, and kurtosis, $K$, plotted as functions of time for the selected stall stage at $r / R=0.382$ at a pitch rate of $\dot{\alpha}=34.28 \ldots . . . . . . .104$

7.8 Separation point peak vorticity histogram for all measurement planes, $r / R=0.19,0.25,0.32$, and 0.38 for pitching rates, $\dot{\alpha}=$ 17.14 (solid) and 34.28 (dashed), with magnitude $\left(\omega^{*}\right)$ on the $x$ axis and frequency of occurrence (\%) on the $y$-axis. . . . . . . 106

7.9 Normailzed POD time coefficients (a) $a_{1} / \sqrt{\lambda_{1}}$ vs. $a_{2} \sqrt{\lambda_{2}}$ and (b) $a_{2} \sqrt{\lambda_{2}}$ vs. $a_{3} \sqrt{\lambda_{3}}$, plotted with color scheme that highlights attached flow in yellow and full stall in green for $r / R=0.252$ at $\dot{\alpha}=34.28 \ldots \ldots \ldots \ldots \ldots$

7.10 Normailzed POD time coefficients (a) $a_{1} / \sqrt{\lambda_{1}}$ vs. $a_{2} \sqrt{\lambda_{2}}$ and (b) $a_{2} \sqrt{\lambda_{2}}$ vs. $a_{3} \sqrt{\lambda_{3}}$, plotted with color scheme that highlights attached flow in yellow and full stall in green for $r / R=0.190 .38$ at $\dot{\alpha}=17.14$ (solid) and 34.28 (dashed) . . . . . . . . . . . . . 110 
7.11 Shear layer origin based on the convective velocity of $0.48 U_{\infty}$ plotted against the fist occurrence of zero vorticity within the separated volume for all planes under the influence of a pitch rate of (a) $\dot{\alpha}=17.14$ and $(b) \dot{\alpha}=17.14$, tracking from attached flow stages (yellow) to full stall (green). . . . . . . . . . . . . . . 113

7.12 Ratio of first shear layer perturbation amplitude $A_{1}$ and maximum amplitude $A_{\max }$ on the $x$-axis plotted against $A_{\max }$ on the $y$-axis, with stall stages mapped from attached flow states (yellow) to full stall (green). . . . . . . . . . . . . . . . . . 115

7.13 Ratio of first shear layer perturbation amplitude $A_{1}$ and maximum amplitude $A_{\max }$ on the $x$-axis plotted against $A_{\max }$ on the $y$-axis, with stall stages mapped from attached flow states (yellow) to full stall (green). . . . . . . . . . . . . . . . 116

7.14 Shear layer maxima and minima on the $y$-axis plotted against the separation point $f(x / c)_{\omega=0}$ on the $x$-axis, with stall cycle following from attached flow (yellow) for full stall (green) at a pitch rate $\dot{\alpha}=17.14$ at $r / R=0.32$ with a least squares fit (black) . . . . . . 118

7.15 Least squares fit of shear layer amplitude maxima and minima plotted on the $y$-axis against the point of separation $f(x / c)_{\omega=0}$ on the $x$-axis for all planes $r / R=0.19-38$, and pitch rates $\dot{\alpha}=17.14$ (solid) and $\dot{\alpha}=34.28$ (dashed). . . . . . . . . . . . . . . . . . . 119

7.16 Separation point peak vorticity histogram for an oscillating OA209 airfoil with magnitude $\left(\omega^{*}\right)$ on the $x$-axis and frequency of occurrence $(\%)$ on the $y$-axis. . . . . . . . . . . . . . . 121 
7.17 Shear layer maxima and minima on the $y$-axis plotted against the separation point $f(x / c)_{\omega=0}$ on the $x$-axis, with stall cycle following from attached flow (yellow) for full stall (green) at a pitch rate $\dot{\alpha}=17.14$ at $r / R=0.32$ with a least squares fit (black) for an oscillating OA209 airfoil. . . . . . . . . . . . . . . . . . 122

7.18 Shear layer origin based on the convective velocity of $0.48 U_{\infty}$ plotted against the fist occurrence of zero vorticity within the separated volume for an oscillating OA209 airfoil. tracking from attached flow stages (yellow) to full stall (green) . . . . . . . . . . . . . . . . 123 


\section{Introduction}

Since the beginning of time the natural world has captured the human imagination. When watching a wave crash or a bird fly one does not need to understand physics to see their natural beauty. The combination of power and grace that are present in these situations can be overwhelming to some, but for others it inspires a deep and insatiable curiosity. The need to understand something in nature is perhaps the greatest strength of the human race. It has allowed for interactions with the world around in ways that are unmatched. Through the application of the knowledge gained by seeking an understanding of the environment, humans have been able to develop agriculture, cure diseases, and even fly.

Over the last century, human flight has transformed from an impossible dream into a wide spread mode of transportation. The concepts that are used to fly across the globe are also being applied to the production of electricity. Weather it is used in a hydroelectric damn, a gas turbine, or a wind mill the harnessing of the power contained within a moving fluid is guided by the same principles that allow birds to fly.

Scientific development has allowed to overcome some of the most fundamental challenges presented by our environment. However, as soon as one problem is solved 
new challenges emerge. One of the most pressing issues faced by the growing global population is striking a balance between meeting the ever increasing demand for energy and the negative environmental impacts that come with it. The use of fossil fuels for that power source has been viable for the past few centuries, however the supply of these materials is finite. Many estimates have been made and debated about when the decline of fossil fuels will begin $[1,2]$. Although such time lines are under debate, there is little doubt about the global impact that will follow. Furthermore, the rise of global climate change which has been directly attributed to the emission of carbon dioxide, one of the bi-products of fossil fuel combustion has motivated an economic shift towards renewable and sustainable energy sources [3]. Of the possible sources for such energy, wind energy has been identified as a resource which can offer a significant contribution to the world's future energy supply [3-5].

One solution to the going need for sustainable energy is wind power. However, as abundant as wind may be there are several challenges that must have yet to be overcome with regard to harvesting and distribution of wind power. Wind turbines operate in the atmospheric boundary layer and are thus subject to strong gradients of velocity and large velocity fluctuations in the form of gusts and atmospheric changes by time of day and season [6]. The presence of atmospheric turbulence causes rapid changes in wind speed which leads directly to unsteady loading and unloading of the turbine blades, the generators and the power transmission system. The impact of seemingly random changes in power, torque, and force on the turbine leads to conservative designs that attempt to mitigate the adverse effects.

From an aerodynamic perspective, rapid changes in wind speed can lead directly to aerodynamic stall. When an airfoil is performing optimally, the aerodynamic 
condition is known as attached flow. When the angle at which the airfoil meets the incoming wind becomes large, the airfoil will pass into a state known as stall. When stall occurs, the lift force that is driving a wind turbine is lost and the power produced by that turbine drops. The transition between attached flow and stall is a process known as separation. For wind turbines, a strong wind gust can cause a turbine blade to pass in and out of stall rapidly. The transition from one stage to the next leads to extreme loading and unloading of the blade. This is called unsteady separation and is often the cause of premature breakdown of wind turbines as well as limiting the performance of the turbine on a daily basis.

Of the remaining unresolved issues in fluid dynamics, one could argue that unsteady flow separation appears high on the list due to the vast number of engineering applications that it directly affects. Understanding and controlling the emergence and persistence of separation would, for example, lead directly to the improved performance of unsteady pipe flow in a water system. It can also greatly improve the lift characteristics of helicopters, effectively reducing their noise and fuel consumption [7]. Yet another example is in the renewable energy sector, where wind turbines, operating under unsteady conditions, experience unsteady separation and frequently leading to unpredictable energy output and considerable fatigue of the turbines components.

When a flow separates, for example over a wing, friction over wing it is separating from increases significantly. This is known as aerodynamic drag. The result of increasing drag is that more force is required to to move the wing through the air. For wind turbines this simply reduces production of the turbine, but the consequences for airplanes can be catastrophic. 


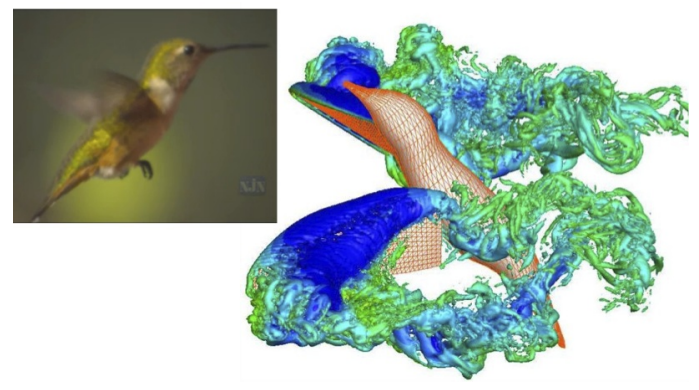

(a) Computational fluid dynamic rendering of Hummingbird flight [8]

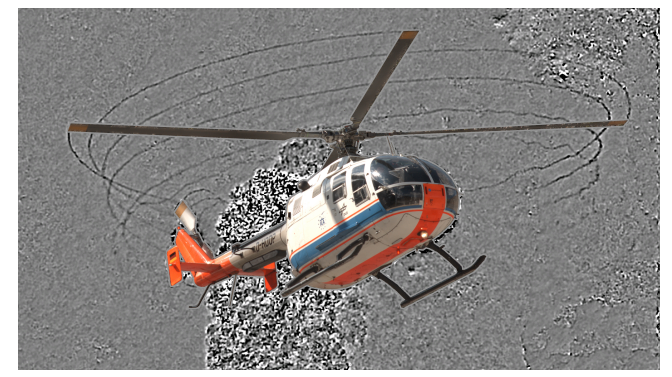

(b) Schlieren photography of helicopter blade vortex shedding by DLR [7]

Figure 1.1: Examples of unsteady separation captured by (a) computational fluid dynamics and (b) Schlieren photography.

Despite the wide spread negative effects unsteady separation has in human made systems, many biological organisms utilize unsteady separation. Through the manipulation of surface vorticity low-Reynolds number flight of hummingbirds and insects becomes possible [8]. Significant evidence in the natural world exists to demonstrate the benefits of unsteady flow separation control, however an adequate formalization of this phenomena remains elusive.

To reach a fundamental understanding of unsteady separation one must begin by examining the boundary layer. Boundary layer theory originated at the beginning of the $20^{\text {th }}$ century when Prandtl [9] developed the idea of a thin region near a surface where a fluids velocity changes from zero at the surface, due to the noslip condition, to the free stream velocity at a sufficient distance away from the wall, where viscous effects are no longer significant. Within the boundary layer, the large velocity gradients in the boundary layer cause the streamwise viscous forces to be of the same order as the convective terms in the Navier-Stokes equations. A consequence of the viscous interaction near the surface is the introduction of circulation and vorticity into the flow. The boundary layer equation is formally 
derived under the limit of $R e \rightarrow \infty$, and therefore makes the assumption that the boundary layer remains sufficiently thin and does not significantly affect the outer inviscid flow. Under these conditions, Prandtl demonstrates that in a steady flow past a two-dimensional streamlined body, flow will separate from the surface where the skin-friction is reduced to zero and/or exhibits a negative gradient. Within a steady incompressible two-dimensional velocity field $u(x, y), v(x, y)$, and $\tau_{w}$ denotes the skin friction along the wall. Then, Prandtl claimed that steady separation will occur at a point, $p=\left(x_{s}, y=0\right)$ if,

$$
\begin{gathered}
\left.\tau_{w}(p)=\nu \rho u_{y}(p, 0)\right)=0, \\
\tau_{w}^{\prime}(p)=\nu \rho u_{x y}(p, 0)<0 .
\end{gathered}
$$

Prandtl's separation conditions give an Eulerian description of boundary layer behavior, which fits well in the case of steady separation [9]. However, accuracy of this condition breaks down in the presence of an adverse pressure gradient acting in the streamwise direction. For many fundamental flow fields and various fluid applications, the occurance of flow separation occurrs due to the effect of a streamwise adverse pressure gradient. On a curved surface, such as an airfoil, there is a non-negligible pressure gradient acting on the flow that impacts boundary layer growth. The thickening of the boundary layer leads to singularity in Prandtl's steady boundary layer solution when the wall shear stress vanishes, first discovered by [10]. The Goldstein singularity was studied simultaneously by Moore [11], Rott [12], and Sears [13], who independently arrived at the conclusion that vanishing wall shear stress and the accompanying flow reversal near a wall do not necessarily guarantee flow separation under unsteady conditions, known now as the MRS criterion. Ac- 
cording to the MRS condition, the separation point instead must occur on the zero vorticity line bisecting a recirculation region. A Lagrangian solution was proposed by [14] to address the issues with Prandtl's boundary layer solution. The Lagrangian approach was found to accurately describe the convergence and ejection of fluid particles from an unsteady boundary. The Lagrangian formulation for the unsteady boundary layer has two advantages: (i) It decouples the motion in the streamwise direction from that in the normal direction, and (ii) There is a definitive criterion for a singularity in the boundary layer equations. The emergence of a Lagrangian treatment for unsteady separation leads to many developments within the field of unsteady separation. Since then three dimensional flows have been shown to separate along lines or manifolds, not isolated points [15-17]. Recently, Surana et al. [18] developed an exact theory of steady flow separation and reattachment on boundaries at rest, which has been extended to treat unsteady separation. These works focus on the connection between the Lagrangian sources of flow separation, [19-21] and the Eulerian fields of skin-friction lines, i.e. the separation lines [18] by analyzing unstable manifolds that emanate from a surface.

In the case of an airfoil, the development of unsteady separation is relatively complex due to the effect of arifoil shape and the effective angle of attack on the surface pressure graident. As shown in Figure 1.2, the surface pressure gradient is divided into two sections at the maximum thickness of the blade measured normal to the inflow. Upsteam of the maximum thickness, flow is accelerating under the influence of a favorable pressure gradient. In this region, the boundary layer is thin and the vorticity is contained within evenly distributed vortices that form near the surface due to shear. Once the flow passes the maximum thickness, the pressure 


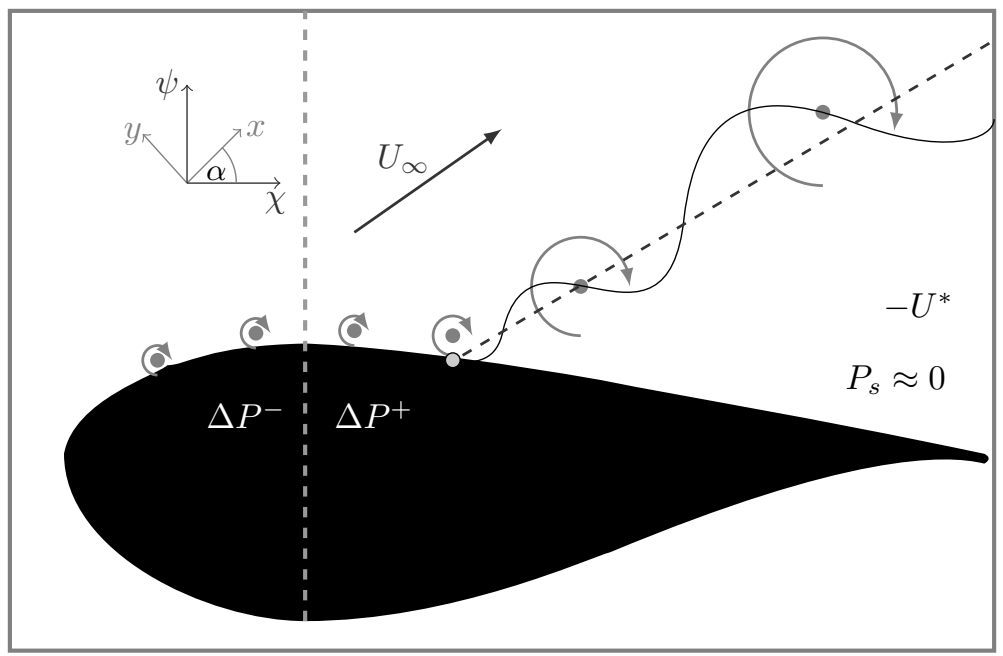

Figure 1.2: Schematic of theoretical unsteady separation over an airfoil. For consistency of presentation, coordinates are rotated from $(x, y)$ to $(\chi, \phi)$ adjust for the angle of attack, $\alpha$, realative to the freestream velocity, $U_{\infty}$. The perturbed shear layer is plotted as a black dashed line emanating from the separation point (grey 'o') with perturbations caused by departing surface vortices (grey ' $\rightarrow$ ') plotted over a black dashed line which represents a steady shear layer without perturbation.

gradient transitions to an adverse pressure gradient which becomes increasingly strong as the angle of attack is increased. The effect of increasing streamwise surface pressure is to force low momentum fluid within the boundary layer upstream leading to a pronounced separation region. In studies by Adrian et al. [22] and Cassle et al. [23], the boundary vorticity field was shown to play a significant role in flow separation. Excessive vorticity leads to the formation of coherent vortices, shown as grey $(\rightarrow)$ in Figure 1.2. As vorticity builds up, it obstructs flow along the surface deflecting the free stream away from the surface increasing the downstream velocity deficit; effectively increasing the boundary layer thickness. At some point, low momentum fluid within the boundary layer is unable to enter the high pressure 
region downstream and is drawn into the low pressure/high momentum outer flow. This leads to flow reversal generated by the vorticity and hence a reduced skin friction. Vortices which form will eventually depart and are convected over the blade surface, shown in Figure 1.2, causing concentrated pressure fluctuations within the flow. Pressure fluctuations act as perturbations in the free shear layer and play a critical role in aerodynamic loading. In an exposition of shear layer vorticity during separation, [24] sought to account for Reynolds number variation of the velocity and length scales which govern the vortex shedding frequency. It was found that the perturbation frequency is influenced by the base pressure, Strouhal number $(S t)$ and the upstream variation of the transition point, as defined by Sato, which depends on temporal changes in the near wake structures [24]. This process is highly unstable, particularly during unsteady changes in the effective angle of attack. The shear layer experiences perturbations developed via Kelvin-Helmholtz instability, which scale with the thickness of the separated flow region, growing in magnitude as they are convected downstream [24]. These perturbations are assumed to take on a wave form and cause the flow above the perturbation apex to accelerate, effectively reducing the pressure above the shear layer, and consequently amplifying the wave. The opposite is true, in the trough of the perturbation, where the fluid which moves slower has a higher pressure and will experience a further increase in pressure. The trough is then forced downwards and increases the amplitude of the wave [25]. The growth of the perturbations and advancement of the shear layer instability towards the leading edge leads to inflections of the shear-layer near to the separation point and abrupt movements of the separation point itself [26].

Despite the advances in the theoretical development of unsteady separation, 
there remains to be a significant gap in the experimental data with respect to the interaction between the vorticity within the boundary layer and its role within the context of unsteady separation. In various engineering applications, the outstanding challenge is to identify the initial location and strength of the separating shear layers [27]. Knowledge of these quantities, can provide critical design criteria to aid in the development of more efficient engineering applications.

Currently, theoretical studies of unsteady separation fall into one of two categories. The first is topological which is widely used theory of vector fields for three dimensional flow separation. Specifically, this is applied to the classification of fixed points in the skin-friction field on a wall and within the velocity of vorticity fields in the flow such as [15]. This type of analysis provides deeper qualitative understanding into even the most complicated flow fields. However, to provide a quantitative separation criteria the knowledge of topological behavior has to be combined with more quantitative analysis [27]. In contrast to topological theory, the second category of theoretical exposition is quantitative and asymptotically accurate in the limit of $R e \rightarrow \infty$, is the interactive boundary layer theory known as the triple-deck theory for steady separation, developed by [28]. This approach treats the interaction between the viscous boundary layer and the inviscid outer flow as a truly coupled system using asymptotic matching to arrive at a complete solution for steady separation, but a full solution in unsteady conditions has yet to be developed [23].

The work in this thesis provides insight into the development of unsteady separation within both Lagrangian and Eulerian frameworks. Through the use of techniques such as proper orthogonal decomposition, variational theory of Lagrangian coherent structures and empirical mode decomposition, a practical understanding 
of unsteady separation is explored. The aerodynamic application that motivates the following experimental campaign is the complex surface flow of a wind turbine blade under unsteady conditions.

\subsection{Unsteady separation applied to wind energy}

Several challenges remain in wind energy harvesting techniques. Foremost is the interaction between the turbines and turbulent inflow present in the atmospheric boundary layer. Atmospheric turbulence creates highly variable operational conditions, which affect the life expectancy of the components and the effective power output of the turbine [29]. In the case of large scale turbine arrays, the operational conditions of individual turbines are often influenced by the wakes of upstream turbines. An improved understanding of the interaction between the incoming wind and the turbines could result in significant gains in turbine efficiency and reduction in the occurrence of fatigue failure [29-33]. One of many improvements being sought is the creation of accurate models for predicting the unsteady aerodynamic loads that a turbine blade experiences under dynamic operational conditions. The aerodynamic system of a wind turbine is considerably complex due to the coupling of the unsteady atmospheric boundary layer and blade rotation [34]. More specifically, the effective velocity that a particular blade section experiences is directly tied to the rotational velocity as well as the incoming wind velocity, thus playing a critical role in the pressure distribution over the surface leading to radially dependent aerodynamic lift and drag characteristics [35].

Aerodynamic performance of an airfoil is often estimated using the blade element momentum (BEM) theory, which assumes two dimensional flow. Additions 
have been made to BEM to account for three-dimensional effects and losses at the tip of finite aerodynamic profiles by [36], known as the Prandtl-Glauert "tip-loss" correction model. BEM is computationally inexpensive and provides a reasonable estimation for fixed aerodynamic profiles while the flow remains attached to the surface. However, in the case of a dynamically pitching or a stalled blade section, aerodynamic characteristics change considerably and are not accounted for when using BEM [37]. Moreover, BEM under-predicts the wind turbine rotor power output under stalled conditions. Wind turbines are often designed to operate under conditions where a significant portion of the turbine blade experiences some degree of stall. In the case of stall-regulated turbines, stall is utilized to limit the maximum power output to prevent generator overload and excessive forces in the blades during extreme wind speeds [38]. However, considerable uncertainty remains in understanding the emergence and reduction of stall in active wind turbines. For example, the combined NREL (Phase II) wind turbine exceeded predictions by $15-20 \%$ [39].

The occurrence of stall on a wind turbine is primarily found in the inboard or near-root region. Its manifestation occurs due to the shape of the root region, which generally possesses a higher thickness to chord ratio leading to a significant adverse pressure gradient down-stream of the location of maximum thickness [40]. Additionally, the effective velocity, $V_{\text {eff }}$, in that region is affected predominantly by the local fluctuations of incoming wind speed. When the effective velocity changes sufficiently, the effective angle of attack may surpass the static stall angle, $\alpha_{s s}$, causing flow separation or stall. Accurately predicting the occurrence and severity of stall is slightly more complicated when introducing rotational effects imparted by the rotor. Experiments by [41] observed that the effects of rotation lead to lift 
coefficients which exceeded BEM predicted values near the root of the rotor blade. Dwyer et al. [42] looked into the effects of rotation on an unsteady boundary layer over a helicopter rotor blade, concluding that improved lift characteristics result from the introduction of coriolis and centrifugal forces which accelerate stalled volumes of air towards larger radial positions. This effect is known as centrifugal pumping. Spanwise flow generated by centrifugal pumping tends to decrease the boundary layer thickness and reduces the effect of the adverse pressure gradient, resulting in the lift coefficient being higher than what would be obtained from wind tunnel measurements on a non-rotating blade [43]. The role of centrifugal pumping on the overall performance of a wind turbine rotor has been investigated extensively $[34,38,39,39,44-47]$. Du et al. were able to demonstrate that the separation point on the blade surface is delayed due to rotation, particularly in the inner half of the typical turbine blade [34]. The inclusion of centrifugal pumping in aerodynamic modeling remains challenging due to the unsteady inflow of an operational wind turbine [48]. However, accurate models that incorporate rotation could lead to a $30 \%$ increase in production according to Sørensen et al. [46].

When combining the unsteadiness of the wind and the resulting changes in the effective angle of attack, the occurrence of stall becomes a dynamic process. Studies on dynamic stall address the time dependent interaction between the potential and viscous flow during a rapid change in angle of attack, where flow separation is delayed to angles of attack beyond the static stall angle, $\alpha_{s s}$, known as stall delay $[41,49-52]$. The extent of stall delay has been found to be dependent upon parameters such as rate of change in the angle of attack, airfoil shape, pitching frequency or gust frequency in the case of wind turbines, Mach number, and the 
Reynolds number [52]. Shih et. al [53] defined unsteady flow development over an airfoil pitching at a constant rate into four successive stages: 1) vortex formation stage, 2) vortex convection stage, 3) stall onset, and 4) full stall stage. Both Reynolds and Carr [52] and Shih et al. [53] found that the process of vortex formation and convection result in a delay of massive flow separation to angles of attack which surpass the static stall angle. The delay of separation causes the lift to increase as the angle of attack increases causing lift overshoot, characteristic of dynamic stall. Theoretical derivations of unsteady separation, conducted by [51], identified that the effective separation points during transition are dependent on the times scales over which they are computed. The computational time scales are estimated analytically and used to approximate the movement of the separation point. Li et al. [54] performed numerical simulations of the NREL phase VI experimental wind turbine finding that Detached Eddy Simulation (DES) was able to predict the performance of the outboard regions with fair accuracy, but was limited at the inboard regions, $r / R<0.3$. Similarly, Xu et al. [55] used simulations which stress the importance of including the rotational and three-dimensional effects in modeling of dynamic stall. Flow dynamics were matched on the outboard sections of the blade, but accurately captureing the dynamic stall behavior in the root section proved difficult. Models based on Kirchhoff-Helmholtz theory, such as the one proposed by $\mathrm{Xu}$ et al. [55], depend directly on the separation point as a function of angle of attack. In this study, measurements over a known airfoil under the influence of a spanwise pressure gradient provide information about the point of transition to stall and flow reattachment as a function of angle of attack and the time scales associated to unsteady separation. 


\section{Theory}

\subsection{Coherent Structure Identification Techniques}

Consequences of unsteady separation stem from vorticity formation and build up over the surface of a pitching arifoil [51]. Vorticity transport is numerically represented by the curl of the Navier-Stokes equation, which in the case of incompressible flow in the absence of non-conservative body forces is reduced to:

$$
\frac{\partial \omega_{i}}{\partial t}+u_{j} \frac{\partial \omega_{i}}{\partial x_{j}}=\omega_{i} \frac{\partial u_{i}}{\partial x_{j}}+\nu \frac{\partial^{2} \omega_{i}}{\partial x_{j}^{2}}
$$

where $u_{i}$ is instantaneous velocity vector, $\omega_{i}$ is instantaneous vorticity and $\nu$ is kinematic viscosity. Equation 2.1 is invariant with respect to Galilean and constant rotation transformation, allowing for a description of vortices and vortex patterns [56]. The pressure term is also eliminated making explicit consideration of pressure unnecessary [57].

Through the physical definition provided by Equation 2.1, it becomes possible to quantify the rotational velocity field within a flow. The accumulation and shedding of vorticity leads to the formation of coherent structures known as vortices. The $\lambda_{2^{-}}$ criterion and the $\Gamma_{2}$-criterion, outlined by [56] and [58], respectively allow for vortex 
detection within a flow field. According to [56], a vortex is identified as regions in a flow field where:

$$
\lambda_{2}\left(\mathbf{S}_{\mathbf{i j}}^{2}+\boldsymbol{\Omega}_{\mathbf{i j}}^{2}\right)<0 .
$$

Here, $\lambda_{2}$ is the intermediate eigenvalue of the tensor, $\left(\mathbf{S}_{\mathbf{i j}}{ }^{2}+\boldsymbol{\Omega}_{\mathrm{ij}}{ }^{2}\right)$, where $\mathbf{S}_{\mathbf{i j}}=$ $1 / 2\left[\partial u_{i} / \partial x_{j}+\partial u_{j} / \partial x_{i}\right]$ is the rate of strain tensor of the velocity field, $u_{i}(x, t)$, and $\boldsymbol{\Omega}_{\mathbf{i j}}=1 / 2\left[\partial u_{i} / \partial x_{j}-\partial u_{j} / \partial x_{i}\right]$ is the antisymmetric rate-of-rotation tensor. Using adiabatic assumptions, this criterion guarantees a local pressure minimum in a twodimensional flow and arises from analyzing the invariants of $\partial u_{i} / \partial x_{j}$. By definition, $\lambda_{2}$ is Galilean invariant, but is inherently a non-objective definition. While the $\lambda_{2}$-criterion is capable of locating a singular vortex, it does not provide a specific location of a vortex axis $[51,57]$.

To locate a specific vortex axis, the $\Gamma_{2}$-criterion outlined by [58] is ideally suited. Letting $\mathbf{x}_{i}$ be a specific point in the measurement domain,

$$
\Gamma_{2}\left(\mathbf{x}_{i}\right)=\frac{1}{M} \sum_{\mathbf{x}_{j} \in S_{i}} \frac{\left[\left(\mathbf{x}_{j}-\mathbf{x}_{i}\right) \times\left(\mathbf{u}_{j}-\overline{\mathbf{u}}_{i}\right)\right] \cdot \mathbf{n}}{\left|\mathbf{x}_{j}-\mathbf{x}_{i}\right| \cdot\left|\mathbf{u}_{j}-\overline{\mathbf{u}}_{i}\right|}=\frac{1}{N} \sum_{\mathbf{x}_{j} \in S_{i}} \sin \left(\Theta_{i j}\right),
$$

where $S_{i}$ is a two-dimensional area surrounding $\mathbf{x}_{i}, M$ is the number of grid points lying in $S_{i}, \mathbf{n}$ is the unit vector normal to the measurement plane, $\mathbf{u}_{i}$ is the velocity vector at point $\mathbf{x}_{i}, \Theta_{i j}$ is the angle formed by the vectors $\left(\mathbf{x}_{j}-\mathbf{x}_{i}\right)$ and $\left(\mathbf{u}_{j}-\overline{\mathbf{u}}_{i}\right)$, and $\overline{\mathbf{u}}_{i}$ is the local mean velocity in the area $P$ surrounding $\mathbf{x}_{i}$, defined as,

$$
\overline{\mathbf{u}}_{i}=\frac{1}{M} \sum_{\mathbf{x}_{l} \in P} \mathbf{u}_{j}
$$

The $\Gamma_{2}$-criterion is a dimensionless scalar function with values ranging from 
$-1 \leq \Gamma_{2} \leq 1$. The location of a possible vortex is indicated by the extrema of $\Gamma_{2}$ and the sense of rotation is indicated by the sign. Vortex detection methods are able to capture the relatively small structures of a instantaneous vorticity field, which is useful for identifying specific stages of aerodynamic stall.

\subsubsection{Proper Orthogonal Decomposition}

In addition to the small scale structures in a flow field, large scale structures define the overall flow characteristics during the various stages of stall and play a significant role in terms of dynamic loading of a turbine blade. To identify the large scales structures present in the dynamic stall process, proper orthogonal decomposition (POD), a statistical technique based on the two-point velocity correlation, can be used to capture and order the energetic structures present within a flow field. When POD is applied to PIV measurements, the technique applied is known as snapshot POD [59]; evaluating images of a flow field at discrete times. In the following analysis, the two-dimensional velocity field, $\mathbf{u}_{i}\left(\mathbf{x}, t^{n}\right)$, is decomposed.

A series of discrete measurements within a two-dimensional flow field can be described by the spatial coordinates $\mathbf{x}_{i}=\left(x_{1}, x_{2}\right)$ and time of sample $n \Delta t$ :

$$
\mathbf{u}_{n}=\mathbf{u}_{i}(\mathbf{x}, n \Delta t), \quad n \in[1,2, \ldots, N]
$$

and $\Delta t$ as the time between snapshots. The total number of snapshots is denoted by $N$. With a large number of samples the two-point correlation tensor is approximated as, 


$$
R\left(\mathbf{x}, \mathbf{x}^{\prime}\right)=\frac{1}{N} \sum_{n=1}^{N} \mathbf{u}_{i}\left(\mathbf{x}, t^{n}\right) \mathbf{u}_{i}^{T}\left(\mathbf{x}^{\prime}, t^{n}\right),
$$

where $\mathbf{x}^{\prime}$ represents the spatial coordinates of another point in the domain. With the two-point spatial correlation tensor as the kernel of the POD eigenvalue problem, it is assumed that $N$ modes can be written in terms of the original data as;

$$
\Phi(\mathbf{x})=\sum_{n=1}^{N} A\left(t^{n}\right) \mathbf{u}_{i}\left(\mathbf{x}, t^{n}\right),
$$

where $\Phi(\mathbf{x})$ is a deterministic field which has the largest projection on the velocity field in a mean-squared sense.

The POD equation in integral form is written as;

$$
\int_{\Omega} R\left(\mathbf{x}, \mathbf{x}^{\prime}\right) \Phi\left(\mathbf{x}^{\prime}\right) d \mathbf{x}^{\prime}=\lambda \Phi(\mathbf{x}) .
$$

By substituting Equations (2.6) and (2.7) into Equation (2.8), the result is an eigenvalue problem of the form:

$$
\mathbf{C A}=\lambda \mathbf{A} .
$$

The coefficient vector from Equation (2.9) is

$$
\mathbf{A}=\left[A\left(t^{1}\right), A\left(t^{2}\right), \ldots, A\left(t^{N}\right)\right]^{T} .
$$

The elements in matrix $\mathbf{C}$ are:

$$
\left.C_{j k}=\frac{1}{N}\left(\mathbf{u}_{i}^{T}\left(\mathbf{x}, t^{j}\right) \mathbf{u}_{(\mathbf{x}}, t^{k}\right)\right), \quad[j, k=1,2, \ldots, N] .
$$


By solving the eigenvalue problem in Equation 2.9, a set of eigenvectors and time coefficients used to calculate the POD modes from Equation 2.7. To arrive at an orthonormal set, the modes are normalized as follow;

$$
\phi^{n}(\mathbf{x})=\frac{\sum_{n=1}^{N} A\left(t^{n}\right) \mathbf{u}_{i}\left(\mathbf{x}, t^{n}\right)}{\left\|\sum_{n=1}^{N} A\left(t^{n}\right) \mathbf{u}_{i}\left(\mathbf{x}, t^{n}\right)\right\|}
$$

The velocity fields are reconstructed using the eigenfunctions of the POD,

$$
\mathbf{u}_{i}\left(\mathbf{x}, t^{n}\right)=\sum_{j=1}^{N} a_{j}\left(t^{n}\right) \phi^{j}(\mathbf{x}) .
$$

Here $a_{j}$ is a set of coefficients that are obtained by back-projecting the set of velocity fields onto the POD modes,

$$
a_{j}\left(t^{n}\right)=\int_{\Omega} \mathbf{u}_{i}\left(\mathbf{x}, t^{n}\right) \phi^{j}(\mathbf{x}) d \mathbf{x}
$$

The two-point spatial correlation tensor, $R_{i j}\left(\mathbf{x}, \mathbf{x}^{\prime}\right)$, is reconstructed using the eigenfunctions and eigenvalues obtained via POD,

$$
R_{i j}\left(\mathbf{x}, \mathbf{x}^{\prime}\right)=\lambda_{n} \phi_{i}^{n}(\mathbf{x}) \phi_{j}^{n}\left(\mathbf{x}^{\prime}\right)
$$

By contracting the reconstructed two-point spatial correlation tensor, $R_{i j}\left(\mathbf{x}, \mathbf{x}^{\prime}\right)$, the total kinetic energy, $E$, in the domain, $\Omega$, is equal to the summation of the eigenvalues, $\lambda_{n}$. Thus, $\lambda_{n}$ represents the contribution of the corresponding eigenmode to the overall energy of the field,

$$
E=\int_{\Omega} \overline{\mathbf{u}_{i}(\mathbf{x}) \mathbf{u}_{i}(\mathbf{x})} d \mathbf{x}=\sum_{n=1}^{N} \lambda_{n}
$$


The overline denotes ensemble averaging of the given quantity. Once calculated, the eigenmodes are sorted in decreasing order, such that the first modes represent the most energetic structures present in a flow field.

POD has previously been used to identify energetic structures within flow fields [60-62], including experimental wind turbine arrays [32] and to reduce the order of turbulence models [63]. Recently, [57] used proper orthogonal decomposition on time resolved PIV data of a pitching airfoil to capture the time scales related to dynamic stall, in particular to determine the onset of dynamic stall.

\subsubsection{Empirical Mode Decomposition}

Identification of the shear layer perturbation characteristics during stall development is pursued through the application of empirical mode decomposition (EMD) developed by [64]. The original empirical mode decomposition is an analytical method used to analyze non-linear and non-stationary signals. The EMD method makes use of an arbitrary time dependent signal, $I(t)$, and partitions it into a number of intrinsic mode functions (IMFs). Each IMF represents a characteristic time scale in the signal, or image of interest in the case of multi-dimensional ensemble empirical mode decomposition. The IMF constitutes an oscillatory mode that allows for both frequency and amplitude modulation. The first step towards finding an IMF is to identify the local extrema of the signal. An IMF must satisfy two conditions: (i) The number of extrema and the number of zero crossings must only differ by at most one, and (ii) The mean value of the upper and lower envelopes is zero. All of the maxima are then connected using a cubic spline to form an upper envelope. The same process is done for the local minima, producing a lower envelope. The 
corresponding envelope contains all data residing within. The mean of the two envelopes is calculated at each time step, denoted $m_{k}$, and the difference between the original data and $m_{k}$ is $h_{k}$ [64],

$$
I(t)-m_{k}(t)=h_{k}(t)
$$

If $h_{k}(t)$ meets the IMF criteria, then $h_{k}(t)$ is the first IMF of the data series, defined as $c_{k}(t)$. However, if $h_{k}(t)$ does not satisfy the requirements of an IMF, it is treated as an intermediate or proto mode function (PMF). To arrive at the 'true' IMF, a 'sifting' process is applied to the PMF. The sifting process separates the time series into fast and slow oscillations, with the first IMF corresponding to the highest frequencies and the last IMF corresponding to the lowest. The sifting process takes the values for $h_{k}(t)$ and treats it as the original signal and the process is repeated until an IMF, $c_{k}(t)$, is found or one of the stopping criteria is met [65]. The IMF is then subtracted from the original signal;

$$
I(t)-c_{k}(t)=r_{k}(t)
$$

and the remaining data, $r_{k}(t)$, is treated with the same method as before. This process is repeated until the residual data set, $r_{N}(t)$, is no longer capable of being decomposed into an IMF, meaning that the residue is either monotonic or does not possess more than one extremum. Arriving at the final form, the data is decomposed as:

$$
I(t)=\sum_{k=1}^{N} c_{k}(t)+r_{N}(t) .
$$


The resulting IMFs from classic EMD often contain oscillations of significantly different amplitudes or oscillations of similar size that are difficult to separate into different modes. The latter is known as mode mixing [66]. To address these issues, [66] made an extension to the method called ensemble empirical mode decomposition $(\overline{E E M D})$, where the overline denotes the average. $\overline{E E M D}$ takes advantage of the dyadic filtering behavior of the EMD by adding white noise to the signal, to populate the whole time frequency spectrum [67]. The $\overline{E E M D}$ algorithm is as follows: generate $I^{i}[t]=I(t)+w^{i}(t)$, where $w^{i}(t)$ is Gaussian white noise, which is used to produce a set of IMFs. This process is repeated to create several sets of intrinsic modes, $\mathrm{IMF}_{k} \mathrm{~s}$, representing $I^{i}(t)$. Then the $k^{t h}$ mode of the original data $I(t), \overline{\mathrm{IMF}}_{k}$, is found by averaging the $\mathrm{IMF}_{k} \mathrm{~s}$ to an ensemble empirical mode. This method is more accurate than the original EMD method and is free of riding waves [66]. Finally, for the treatment of two-dimensional data or images, the $\overline{E E M D}$ method is extended to reveal the characteristic temporal and spatial scales by use of the multidimensional ensemble empirical mode decomposition ( $\overline{M E E M D})$ [68]. This method assumes that a two-dimensional scalar field, $I(m, n)$, can be decomposed into a finite number of multi-dimensional intrinsic mode functions (mIMFs) which represent the texture of different frequencies or scales within the field. The $\overline{M E E M D}$ sifting is similar to the one-dimensional $\overline{E E M D}$, however a mIMF is acquired using a multidimensional sifting process, which treats the data set as a surface [69,70]. During this process, neighboring windows are used to find local extrema. The value of each extremum must be strictly higher (maximum) or lower (minimum) than the values for the neighboring windows. Herein the $\overline{M E E M D}$ technique is utilized for identifying spatial scales within the time resolved PIV snapshots, however for simplicity 
the results will be discussed as EMD modes for the remainder of the manuscript.

\subsection{Lagrangian Coherent Structures}

Recently, Surana et al. [18] developed an exact theory of steady flow separation and reattachment on boundaries at rest, which has been extended to treat unsteady separation. These works focus on the connection between the Lagrangian sources of flow separation [19-21], and the Eulerian fields of skin-friction lines, i.e. the separation lines. Surana et al. [18] therefore analyzed unstable manifolds that emanate from a surface. The unstable manifolds are lines or surfaces along which fluid particles are either attracted to or ejected from the boundary [18]. The method for identification of these manifolds is given by Farazmand et al. [71] and Shadden et al. [72] as the identification of Lagrangian Coherent Structures (LCS) within Eulerian velocity fields. To be considered an LCS, the material line must have sufficient size to have visible impact and act as a transport barrier; it must also translate with the flow to act as an observable core of evolving Lagrangian patterns. Also, an LCS should be locally the strongest attraction, repulsion or shearing in the flow, which is essential to differentiate the LCS from nearby material lines of the same stability type [71].

Following the notations and definitions of [73], for the case of two-dimensional flows the dynamical system is taken to have the form:

$$
\dot{\mathbf{V}}=\mathbf{u}_{\mathbf{i}}(\mathbf{x}, t)
$$

Lagrangian coherent structures are time dependent surfaces within a dynamic 
flow field. The LCS are considered the skeleton of flow patterns. The approach, defined by [71], identifies strain lines that are either attracting lines or repelling material lines within a flow field during finite time period. The algorithm used herein captures hyperbolic LCSs as smooth material lines, which are obtained as trajectories of an autonomous ordinary differential equation for the tensor lines of the Cauchy-Green strain tensor field.

If $V_{0}=\mathbf{V}\left(t, t_{0}, \mathbf{x}_{0}\right)$ denotes the trajectory of $V$ passing through point $\mathbf{x}_{0}$ at time $t_{0}$ the flow map is defined as;

$$
\mathbf{F}_{t_{0}}^{t}=\mathbf{V}\left(t, t_{0}, \mathbf{x}_{0}\right)
$$

This is as smooth as the vector field $\mathbf{u}_{i}$ enabling the definition of the CauchyGreen strain rate tensor field to be:

$$
\mathbf{C}_{t_{0}}^{t_{0}+T}\left(\mathbf{V}_{0}\right)=\left(\nabla \mathbf{F}_{t_{0}}^{t}\left(\mathbf{V}_{0}\right)\right)^{*}\left(\nabla \mathbf{F}_{t_{0}}^{t}\left(\mathbf{V}_{0}\right)\right)
$$

with $\left.\nabla \mathbf{F}_{t_{0}}^{t}\left(\mathbf{V}_{0}\right)\right)$ denoting the Jacobian of $\left.\mathbf{F}_{t_{0}}^{t}\left(\mathbf{V}_{0}\right)\right)$, and the asterisk, denoting the matrix transpose. The tensor $\mathbf{C}_{t_{0}}^{t_{0}+T}$ is symmetric and positive definite, and hence admits two real positive eigenvalues and orthogonal real eigenvectors. The eigenvalues $\lambda_{i}$ and corresponding eigenvectors $\xi_{i}$ of the tensor $\mathbf{C}_{t_{0}}^{t_{0}+T}$ are defined by the relations:

$$
\begin{gathered}
\mathbf{C}_{t_{0}}^{t_{0}+T}\left(\mathbf{V}_{0}\right) \xi_{i}\left(\mathbf{V}_{0}\right)=\lambda_{i}\left(\mathbf{V}_{0}\right) \xi_{i}\left(\mathbf{V}_{0}\right) \\
\left|\xi_{i}\left(\mathbf{V}_{0}\right)\right|=1, \quad i=1,2
\end{gathered}
$$




$$
0<\lambda_{1}\left(\mathbf{V}_{0}\right) \leq \lambda_{2}\left(\mathbf{V}_{0}\right)
$$

The dependence of $\lambda_{i}$ and $\xi_{i}$ on $t_{0}$ and $T$ is suppressed for notational simplicity. If the flow is incompressible, the eigenvalues of $\mathbf{C}_{t_{0}}^{t_{0}+T}$ also satisfy $\lambda_{1}\left(\mathbf{V}_{0}\right) \lambda_{2}\left(\mathbf{V}_{0}\right)=1$ for any $\mathbf{V}_{0} \in \mathbf{u}_{i}(\mathbf{x}, t)$.

The variational theory presented by [73], searches for material lines surfaces that act as organizational centers of observed trajectory patterns. Such surfaces are distinguished by attracting or repelling of nearby trajectories at the highest rates locally in the flow.

The sufficient and necessary condition for a LCS in two-dimensional flow, considers a compact material line $M(t) \subset \mathbf{u}_{i}(\mathbf{x}, t)$ evolving over the time interval $\left[t_{o}, t_{o}+T\right]$. Then $M(t)$ is a repelling LCS over $\left[t_{o}, t_{o}+T\right]$ if an only if all of the following conditions hold for all initial conditions $V_{o} \in M(t)$ :

1. $\lambda_{1}\left(V_{o}\right) \neq \lambda_{2}\left(V_{o}\right)>1$

2. $\left\langle\xi_{2}\left(V_{o}\right), \nabla^{2} \lambda_{2}\left(V_{o}\right) \xi_{2}\left(V_{o}\right)\right\rangle<0$

3. $\xi_{2}\left(V_{o}\right) \perp M\left(t_{o}\right)$

4. $\left\langle\nabla \lambda_{2}\left(V_{o}\right), \xi_{2}\left(V_{o}\right)\right\rangle=0$

Condition 1 ensures that the normal repulsion rate is larger than the tangential stretch due to shear along the LCS. Conditions 3 and 4 together guarantee that along the repulsion rate attains a local extremum along the LCS relative to all close material lines. Finally, condition 2 ensures that this extremum is a strict local maximum.

Computationally, changes are made to condition 1 in order to utilize finite velocity fields produced by PIV. However, the principal values from theorem 1 remains intact, details of these computational adjustments can be found in [71]. 


\section{Experimental Setup}

The PIV measurements are taken at DLR Göttingen in the 2x1.8 $\mathrm{m}^{2}$ recirculating wind tunnel. For all of the experiments, the inflow velocity is set at $40 \mathrm{~m} / \mathrm{s}$ with a turbulence intensity of less than $1 \%$. The experimental model is a 1:63 scaled model of the NREL 5MW wind turbine blade with a span of $1.05 \mathrm{~m}$. The model is manufactured from solid aluminum to minimize deflections during the experiment. To maximize the chordwise Reynolds number $\left(R e_{c}\right)$, the chord-to-span ratio is doubled while the thickness-to-chord ratio and spanwise profile locations are maintained to preserve the geometric influence on the spanwise pressure distribution. For all of the experiments conducted, the free stream velocity is set to $U_{\infty}=40 \mathrm{~m} / \mathrm{s}$ and the turbulence intensity is less than 1\%. Velocity field measurements are taken near the root section at a radial locations of $r / R=0.187,0.252,0.317$, and 0.382 , with $\delta r / R=0.065$ between adjacent planes. At these locations the chord, $c$, ranges from $15.1 \mathrm{~cm}$ to $13.9 \mathrm{~cm}$, where the chord based Reynolds number ranges from $R e_{c}=413280,424480,401520$, and 390320, respectively. To recreate the boundary layer effects over a rotating wind turbine blade, the twist of the blade is adjusted to match the peak pressure gradient, thus the dynamic pressure is calculated using XFOIL. From aerodynamic theory, it can be shown that the pressure distribution 
of a rotational blade is proportional to the dynamic pressure, $P_{d}$,

$$
P_{d}=\frac{1}{2} \rho\left(\left(U_{w}-\tilde{u}\right)^{2}+(\Psi r+\tilde{v})^{2}\right)
$$

where $\rho$ is the density of the air, $U_{w}$ is the upstream wind speed, $\tilde{u}$ is the instantaneous streamwise induced velocity, $\Psi$ is the rotational velocity of the rotor, $r$ is the radial position, and $\tilde{v}$ is the induced instantaneous velocity tangential to the plane of rotation. The dominant terms in the dynamic pressure equation are the incoming wind speed and the rotational velocity. From a cross-sectional prospective, the dynamic pressure changes with radial position leading to $P_{d} \propto r^{2}$.

Overcoming the technical challenges involved in collecting time resolved flow measurements over a rotating surface, the model is designed to create a peak pressure distribution that follows the $P_{d} \propto r^{2}$ relationship by changing the twist. The rotational pressure distribution is estimated using XFOIL with an effective velocity that results from a steady inflow velocity of $U_{w}=15 \mathrm{~m} / \mathrm{s}$ and a tip-speed-ratio of $T S R=7$ for each aerodynamic profile. The resulting pressure distribution for an experimental NREL 5MW wind turbine blade is shown in Figure 3.1(a). Then using XFOIL, the peak pressure over the respective aerodynamic profiles is calculated for angles of attack ranging from $\alpha=0-18^{\circ}$. The amount of twist for each section is selected by requiring that the maximum pressure gradient ensures that the radial pressure distribution is large enough to play a role in the stall dynamics. The functional pressure gradient along the span is maximized with a twist of $0-18^{\circ}$, shown in Table 3.1. The resulting radial distributions for peak pressure, pressures at $x / c=0.25$, and required angles of attack are presented in Figures $3.1(a),(b)$, and $(c)$, respectively. 


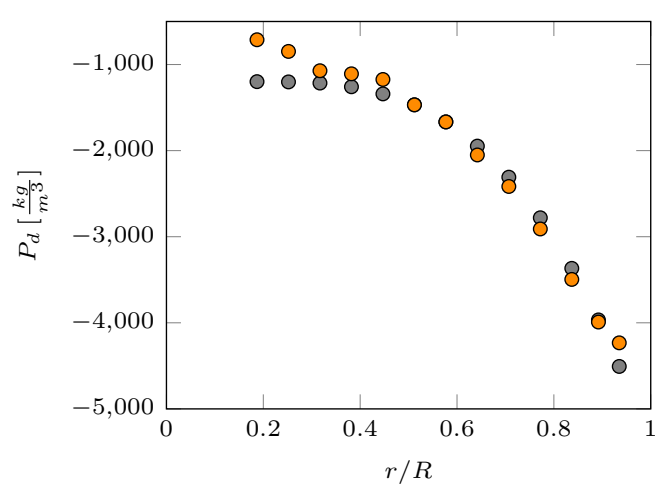

(a)

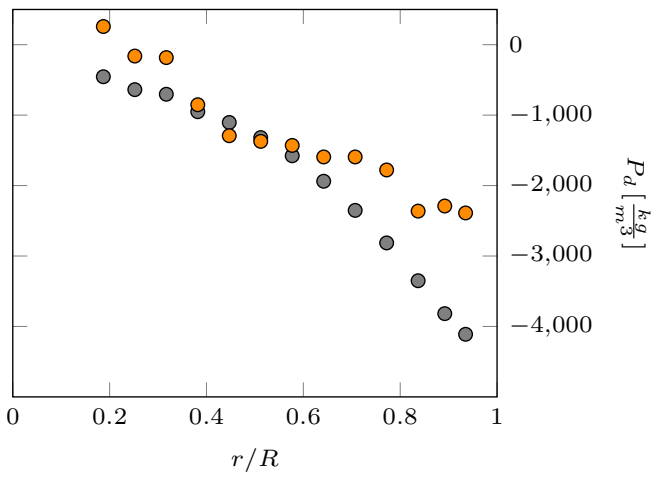

(b)

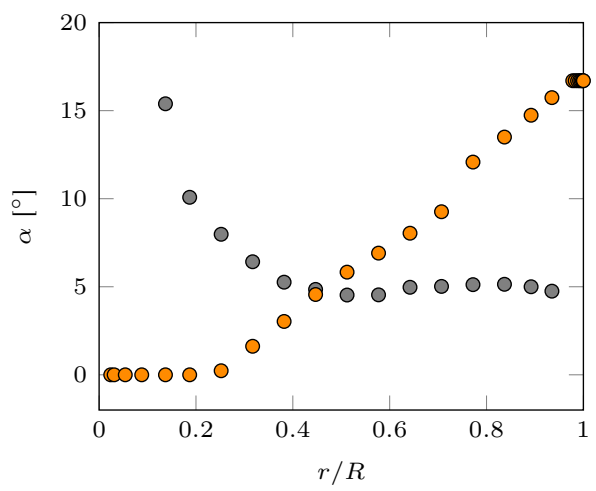

(c)

Figure 3.1: XFOIL based $(a)$ peak and $(b) x / c=0.25$ pressure distributions, $P_{d}$, for an operational (grey) NREL 5MW wind turbine under steady wind speeds of $U_{w}=15 \mathrm{~m} / \mathrm{s}$ plotted next to the estimated peak pressure for the experimental (orange) model blade under steady free-stream speeds of $U_{\infty}=40 \mathrm{~m} / \mathrm{s}$, (c) experimental design (orange) vs. operational (grey) angles of attack plotted as a function of normalized radial position, $\alpha(r / R)$.

For the experimental model based on the NREL 5MW experimental turbine blade, the distribution of aerodynamic profiles in terms of radial position, $r / R$, are given in Table 3.1 along with the ratio of the chord length to radial position, $c / R$, and twist angles $\phi$.

During the experiments, the blade is pitched dynamically using a stepper motor located outside of the wind tunnel to simulate a change in the effective angle of 


\begin{tabular}{|l|c|c|c|}
\hline Profile & $\mathrm{r} / \mathrm{R}$ & $\mathrm{c} / \mathrm{R}$ & Twist $\left(\phi^{\circ}\right)$ \\
\hline DU 99-W-405 & 0.187 & 0.1476 & 0.00 \\
\hline DU 99-W-350 & 0.252 & 0.1516 & 2.300 \\
\hline DU 99-W-350 & 0.317 & 0.1434 & 2.475 \\
\hline DU 97-W-300 & 0.382 & 0.1394 & 4.900 \\
\hline DU 91-W2-250 & 0.447 & 0.1312 & 6.525 \\
\hline DU 91-W2-250 & 0.512 & 0.1230 & 6.925 \\
\hline DU 93-W-210 & 0.577 & 0.1148 & 7.076 \\
\hline DU 93-W 210 & 0.642 & 0.1066 & 7.950 \\
\hline NACA 64-618 & 0.707 & 0.0984 & 9.250 \\
\hline NACA 64-618 & 0.772 & 0.0902 & 12.375 \\
\hline NACA 64-618 & 0.837 & 0.0820 & 15.000 \\
\hline NACA 64-618 & 0.892 & 0.0738 & 16.700 \\
\hline NACA 64-618 & 0.935 & 0.0696 & 18.600 \\
\hline NACA 64-618 & 0.99 & 0.0450 & 18.600 \\
\hline
\end{tabular}

Table 3.1: Aerodynamic profiles with normalized radial location, $r / R$, normalized chord length, $c / R$, and twist angle, $\phi$.

attack, as to mimic a gust encounter. To avoid unwanted flow behavior near the root, the motor mounting plate and blade base, which is extended by $10 \mathrm{~cm}$, are covered by an aerodynamic housing. The total blockage created by the blade and the motor mount housing is less than $7.3 \%$. The blade is pitched at two different pitch rates, $\dot{\alpha}=17.34$ and 34.68 , recreating the gust phenomena with spatial scales that extend for $1 D$ and $2 D$ of the rotor, respectively.

The PIV laser system includes a dual cavity, diode pumped Nd:YLF laser $(\lambda=527 \mathrm{~nm})$ with a pulse energy of $30 \mathrm{~mJ}$ per pulse at $1 k H z$. The PIV planes are located at $h_{p}=0.3 \mathrm{~m}, 0.44 \mathrm{~m}, 0.51 \mathrm{~m}$, and $0.58 \mathrm{~m}$ above the floor of the wind tunnel test section. The blade motion and the time resolved PIV are synchronized such that individual measurement series cover $0.5 \mathrm{~s}$ prior to the initial motion of the blade and after the motion cycle is complete ensuring full coverage of the stall 


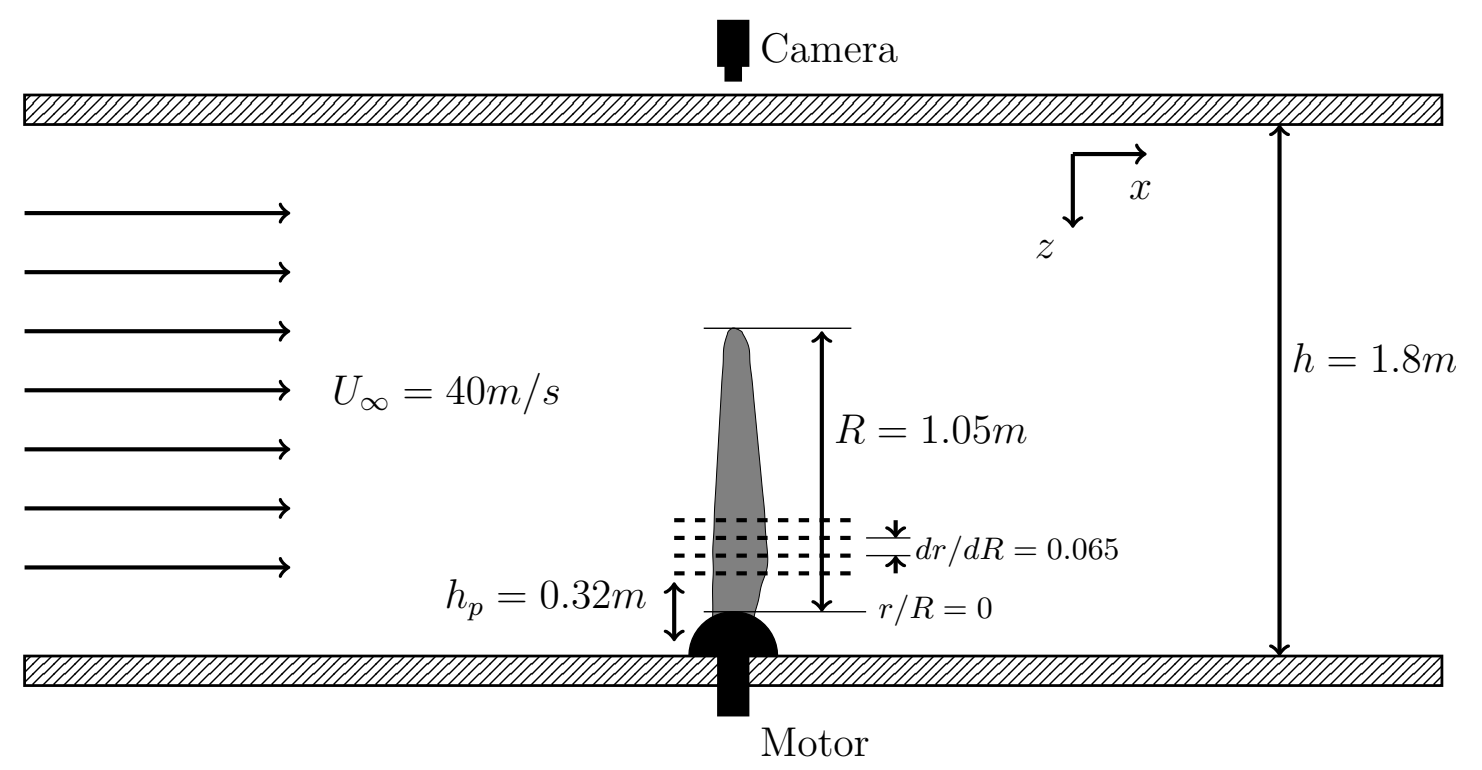

Figure 3.2: Experimental setup: Model turbine blade stands vertically in the $2 \times 1.8 \mathrm{~m}^{2}$ test section, free stream velocity $U_{\infty}=40 \mathrm{~m} / \mathrm{s}$, measurement plane height set to $0.37 m \leq h_{p} \leq 0.58 m, d r / d R=0.065$ between adjacent planes, camera and pitching motor located directly above and below the rotor blade, respectively.

cycle. Time-resolved PIV (TR-PIV) data is collected using a high-speed camera with an acquisition rate of $1 k H z$. The camera is placed directly above the blade just outside of the test section perpendicular to the primary wind direction along the pitching axis of the blade. The time series is collected at the full camera resolution of $1024 \mathrm{px} \times 1024 \mathrm{px}$. The measurement window is $254 \mathrm{~mm} \times 205 \mathrm{~mm}$ with a minimum spatial resolution of $4.5 \mathrm{px} / \mathrm{mm}$. The PIV images were processed using an interrogation window size of $32 \times 32$ and an overlap of $70 \%$ yielding a grid spacing of $7 p x$ or $1.4 \mathrm{~mm}$ which is less than $0.008 \mathrm{c}$. The interrogation window size is minimized to ensure an acceptable signal-to-noise ratio. The velocity fields are rotated into the airfoil reference system, with the $x$-axis along the chord and $y$-axis perpendicular to the chord prior to the analysis. The data acquisition is synchronized with 
the pitching motion allowing for direct connection between the PIV images and the angle of attack. The uncertainty is approximately $3 \%$ of the free stream velocity. Further uncertainty sources are highlighted in [74] and [57].

To capture the three-dimensional effects of unsteady separation, the experimental scope of this project includes PIV images along four spanwise planes at two pitch rates for each of the spanwise positions, as highlighted in table 3.2. By doing so, the dependence of the identified time scales and physical mechanisms are determined for the varied aerodynamic profiles.

\begin{tabular}{|c|c|c|c|c|c|}
\hline Experiment & Profile & $\mathrm{r} / \mathrm{R}$ & $\mathrm{c} / \mathrm{R}$ & Twist $(\phi)$ & Pitch Rate $(\dot{\alpha})$ \\
\hline 1 & DU 99-W-405 & 0.187 & 0.1476 & 0.00 & 17.14 \\
\hline 2 & DU 99-W-405 & 0.187 & 0.1476 & 0.00 & 34.28 \\
\hline 3 & DU 99-W-350 & 0.252 & 0.1516 & 2.300 & 17.14 \\
\hline 4 & DU 99-W-350 & 0.252 & 0.1516 & 2.300 & 34.28 \\
\hline 5 & DU 99-W-350 & 0.317 & 0.1434 & 2.475 & 17.14 \\
\hline 6 & DU 99-W-350 & 0.317 & 0.1434 & 2.475 & 34.28 \\
\hline 7 & DU 97-W-300 & 0.382 & 0.1394 & 4.900 & 17.14 \\
\hline 8 & DU 97-W-300 & 0.382 & 0.1394 & 4.900 & 34.28 \\
\hline
\end{tabular}

Table 3.2: Experimental series based on aerodynamic profiles at normalized radial location, $r / R$, normalized chord length, $c / R$, and twist angle, $\phi$ for two pitch rates $\dot{\alpha}$. 


\section{Proper orthogonal decomposition of unsteady separation}

Here, the use of time resolved PIV provides information such as separation point location as a function of angle and time scales of separation and reattachment on the inboard sections of wind turbine blade. Effects of rotation are replicated by a radially dependent dynamic pressure distribution over a non-rotating finite blade, induced via geometric twist, and unsteady effects are introduced through active pitching of the blade. Velocity fields are analyzed using vortex identification techniques showing the characteristic features of the different stall stages.

Figure 4.1 gives a presentation of the experimental results for velocity, 4.1(a), and vorticity, 4.1(b), that are used in the following sections. The DU 99-W-350 profile is plotted on normalized axes where chord-wise $(x)$ and azimuthal $(y)$ directions are normalized by the chord length. Velocity vectors represent the instantaneous velocity, $\tilde{\mathbf{u}}_{i}$, normalized by the free-stream velocity, $U_{\infty}$. Vorticity presented in figure 4.1(b), is normalized by $c / U_{\infty}$. Using zero contours of the $\lambda_{2}$-criterion and the $\Gamma$-criterion, the sizes and locations of vortices are identified, respectively. Information provided by the TR-PIV yields a detailed description of the locations and size of vortices that form and are convected over the blade surface throughout a stall cycle. 


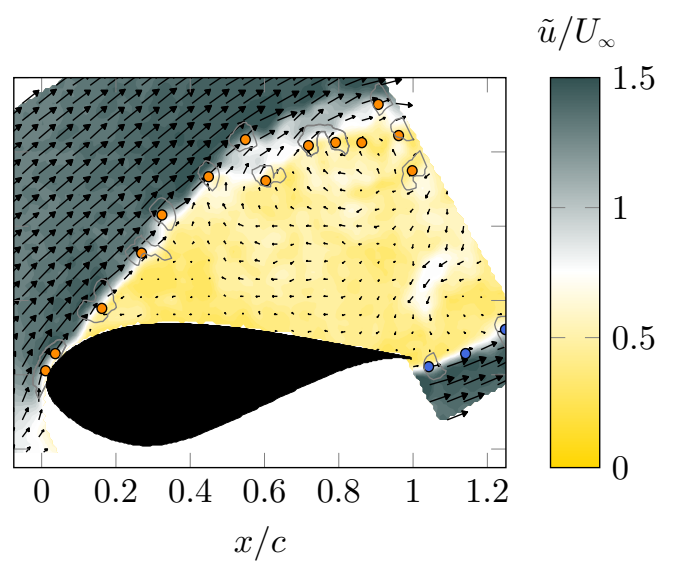

(a) $\alpha=21.81^{\circ}, \tau=50.40$

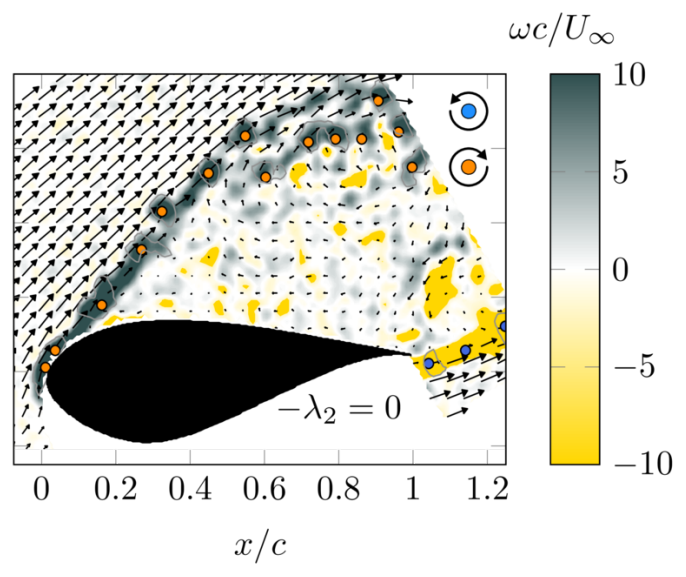

(b) $\alpha=21.81^{\circ}, \tau=50.40$

Figure 4.1: PIV snapshot including a) velocity field (vector map) and b) corresponding vorticity field, contours of $\lambda_{2}=0$ (gray), and vortex location and sense of rotation (clockwise/negative in orange and counterclockwise/positive in blue).

For a radial position of $r / R=0.32$ the NREL 5MW experimental blade profile is a $D U 99-W-350$, with $R$ as the total blade radius. At this location the blade has a twist of $\phi=2.47^{\circ}$ and a chord length of $c=14.3 \mathrm{~cm}$. The $D U 99-W-350$ profile is plotted on normalized axis where chord-wise $(x)$ and azimuthal $(y)$ directions are normalized by the chord length. Velocity vectors represent the instantaneous velocity, $\tilde{\mathbf{u}}_{i}$, normalized by the free-stream velocity, $U_{\infty}$. Using zero contours of the $\lambda_{2}-$ criterion, from Equation (2.2) and the $\Gamma_{2}-$ criterion, from Equation (2.3), the sizes and locations of vortices are identified, respectively. The information provided by the combination of these techniques allows for a detailed description of the stall phenonmena as it develops throughout the pitching cycle. Through an quantitative description of the vortex core locations, the angle of the shear layer, represented by $\beta$, and the corresponding stall area are captured as a function of angle of attack. 
Identified vortex cores, which are used to capture $\beta$, are drawn as small circles and the color surrounding the identified core denotes rotational direction, blue being counterclockwise and orange for clockwise. The second quantity, $\bar{d}_{v}$, is the average size of the vortices, which is characterized by contours of $\lambda_{2}=0$ in that region. These quantities are captured in each image and are tracked as a function of angle of attack.

Following the description of a full stall cycle based on the instantaneous velocity fields, continuity is presented during the stages of the stall cycle. Next, POD is applied to the velocity measurements to evaluate the temporal evolution of the energetic structure of the flow field during the pitching cycle. From the POD time coefficients, times scales for transition are acquired and angles of attack of separation and reattachment are identified. Finally, the velocity fields are reconstructed using the POD modes.

\subsection{Time resolved stall behavior - Vortex identification}

During operation, a turbine experiences fluctuating wind otherwise called gusts. For wind turbines, the variation in wind velocity results in sudden changes in angle of attack. Depending on their strength, gusts can drive the flow over the surface through all of the stall stages. Figure 4.2 shows the motion profile used to recreate the change in angle of attack resulting from a sustained accelerating-decelerating gust. The plot shows dimensionless time $\left(\tau=t_{i} / t_{m}\right)$ on the $x$ axis, where $t_{i}=\left(t_{n}-t_{0}\right)$ is the interval in time between the particular measurement at $t_{n}$. The initial motion begins at $t_{o}$ and $t_{m}$ is the time for one full pitching cycle. The pitching cycles is plotted as the angle of attack as a function of time, $\alpha(\tau)$, on 


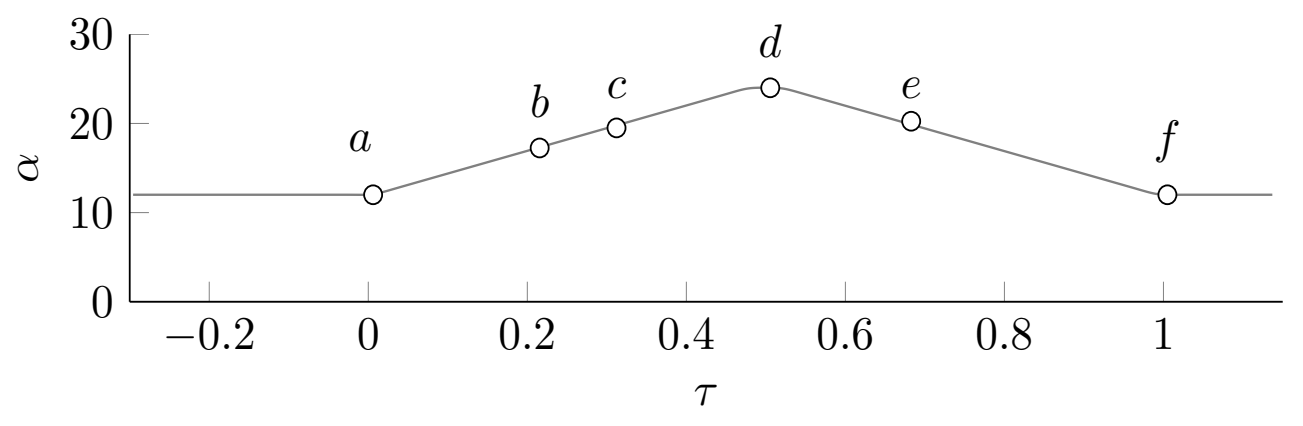

Figure 4.2: Angle of attack $(\alpha)$ vs. time $(\tau)$

the $y$-axis. For this case, the blade pitches from $12^{\circ}-24^{\circ}-12^{\circ}$, over a period of $t_{m}=1.783 \mathrm{~s}$ as shown in Figure 4.2.

In Figure 4.3, the time resolved shear layer angle, $\beta$, and the average vortex size, $\bar{d}_{v}$, are plotted over the motion profile, shown in gray. Times $(a-f)$ are selected as examples of angles of attack in the pitching cycle that capture the stages of the stall cycle and are visually represented in Figure 4.4(a-f). The PIV snapshots, in Figure 4.4, show normalized velocity contours plotted on the same axis as Figure 4.1. On each plot, the vortex cores and zero contours of $\lambda_{2}$ are plotted to aid in the visualization of the stall envelope.

Prior to time $(a), \beta$ holds its smallest and relatively constant value as a function of time. During this period, $\bar{d}_{v}$ demonstrates time dependent fluctuations prior to the beginning of motion which, in combination with the observed behavior of $\beta$, indicates growth and shedding of vortices due to a slight trailing edge stall which is seen in Figure 4.4(a). In Figure 4.4(a), the flow exhibits characteristics of attached flow. A build up of vortices is noted over the surface of the blade section near the 


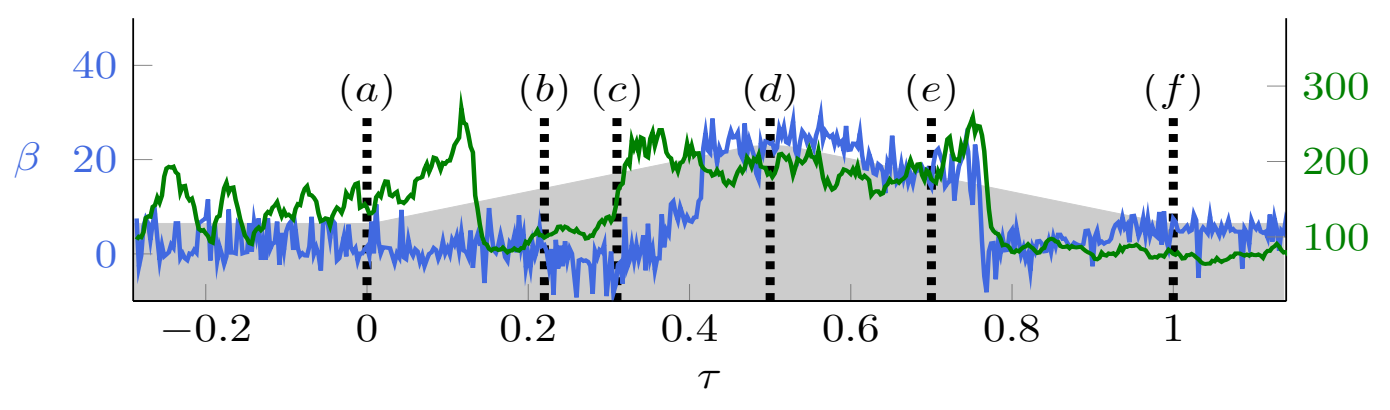

Figure 4.3: Angle of attack, $\alpha(\tau)$ from $12^{\circ}-24^{\circ}-12^{\circ}$ (gray) plotted with vortex separation angle $\beta$ (blue), and vortex size $\bar{d}_{v}$ (green) as functions of time $(\tau)$ during a dynamic pitching cycle

trailing edge. Vortices are present over less than one third of the chord, but the proximity of the shear layer to the airfoil surface is such that the vortices appear equidistant and do not interact.

After time (a), when the blade begins to pitch upwards from $\alpha(0)=12^{\circ}, \beta$ and $\bar{d}_{v}$ diverge. From time (a) in Figure 4.4(a), $\beta$ remains relatively unchanged as the shear layer remains close to the surface just beyond this angle of attack. As $\alpha(\tau)$ increases, the size of the vortices, $\bar{d}_{v}$, also increases, as shown in Figure 4.3. Fluctuations in $\bar{d}_{v}$ reach a peak value before dropping to a minimal value prior to time $(b)$ at $\tau=0.124$. The peak in the average vortex area is a result of a temporary emergence of trailing edge stall, shown in Figure 6.1(a), which remediates prior to the initialization of the stall cycle, shown in Figure 6.1(b). This stall growth indicates that for this section of the rotor blade, the formation trailing edge stall is in fact possible at very low angles of attack, in this case $\alpha(0.124)=14.7^{\circ}$.

During attached flow the large acceleration over the leading edge is accompanied by a strong pressure gradient, which plays a critical role downstream of the leading edge. 


$\begin{array}{llll} & 0 & 0.5 & 1 \\ \tilde{u} / U_{\infty}\end{array}$

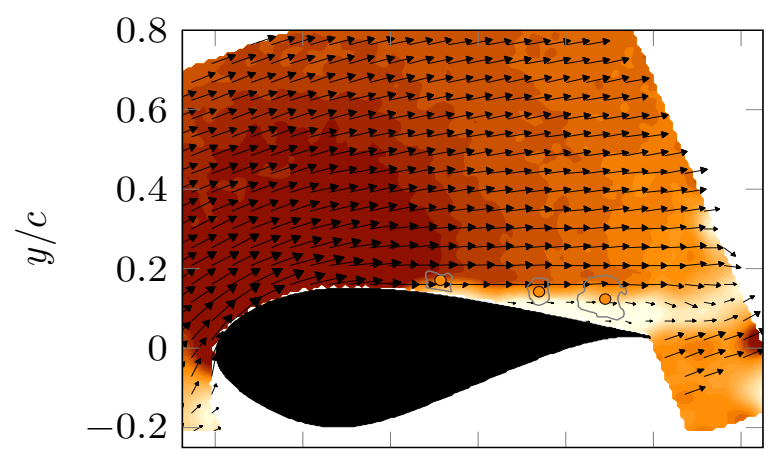

(a) Attached Flow, $\alpha(\tau)=12^{\circ}$

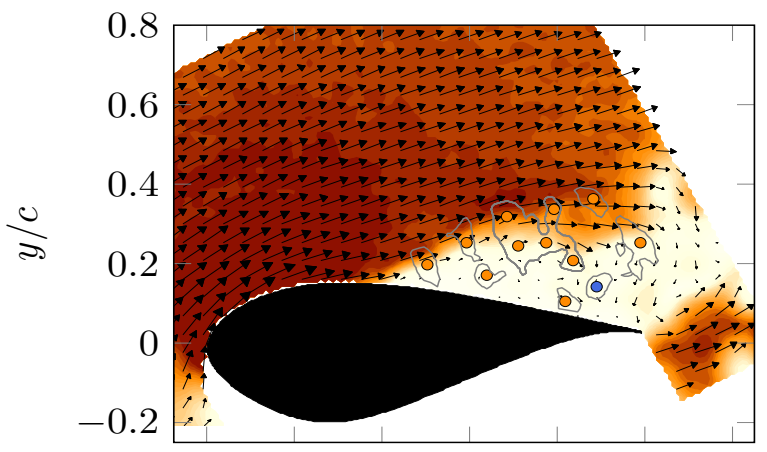

(c) Stall Development, $\alpha(\tau)=20.4^{o}$

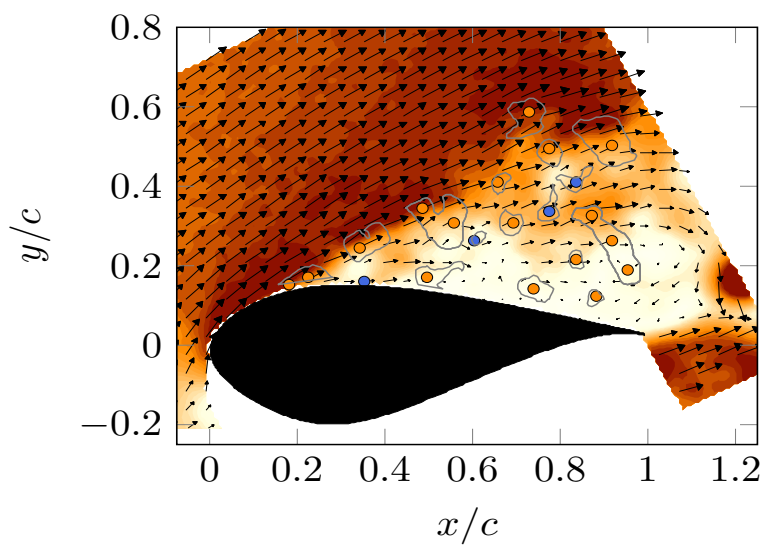

(e) Reattachment, $\alpha(\tau)=20.5^{\circ}$

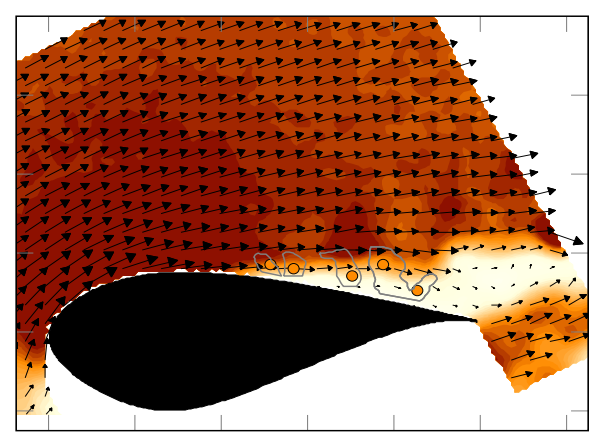

(b) Stall Initialization, $\alpha(\tau)=19.6^{\circ}$

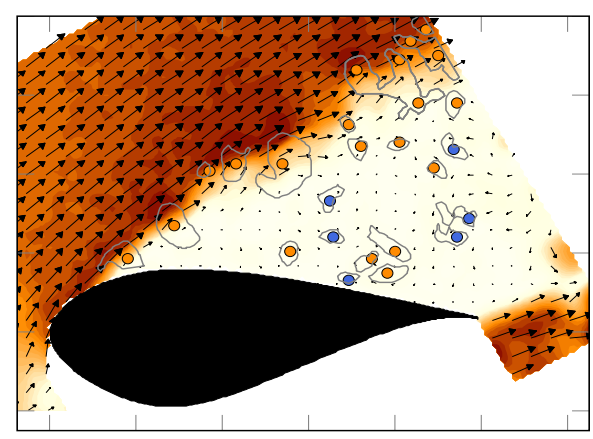

(d) Full Stall, $\alpha(\operatorname{tau})=24^{\circ}$

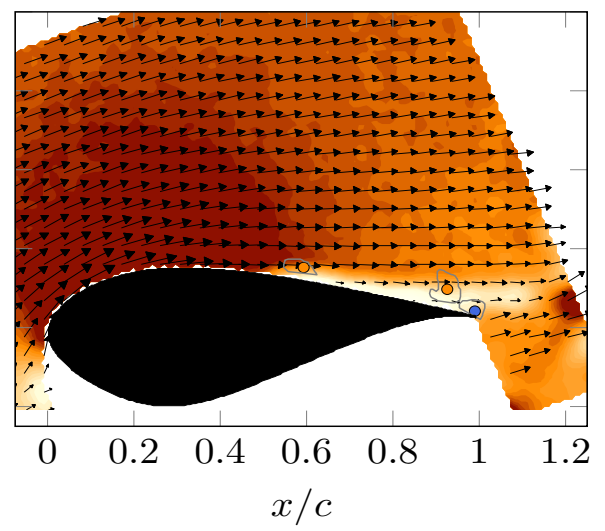

(f) Reattached Flow, $\alpha(\tau)=12^{\circ}$

Figure 4.4: Normalized velocity, $\tilde{u}_{i} / U_{\infty}$, contours and vector fields of instantaneous PIV snapshots, plotted with normalized $x / c$ and $y / c$ coordinates, over a dynamically pitching DU $99-W-350$ cross-section from $\alpha(\tau)=12^{\circ}-24^{\circ}-12^{\circ}$. Vortex areas defined by $\lambda_{2}=0$ contours (gray) surround vortex cores as identified by $\Gamma_{2}-$ criterion which give rotational sense (clockwise in orange and counter-clockwise in blue). 


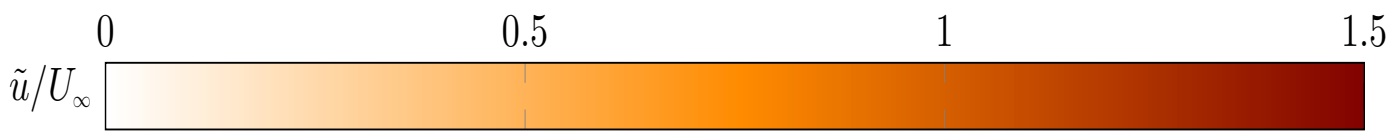

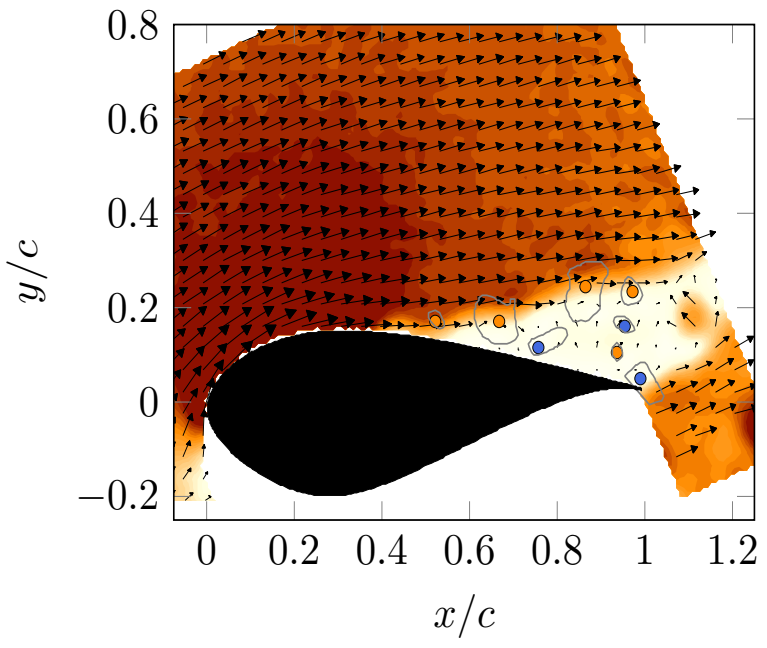

(a) $\alpha=14.7^{\circ}, \tau=0.124$

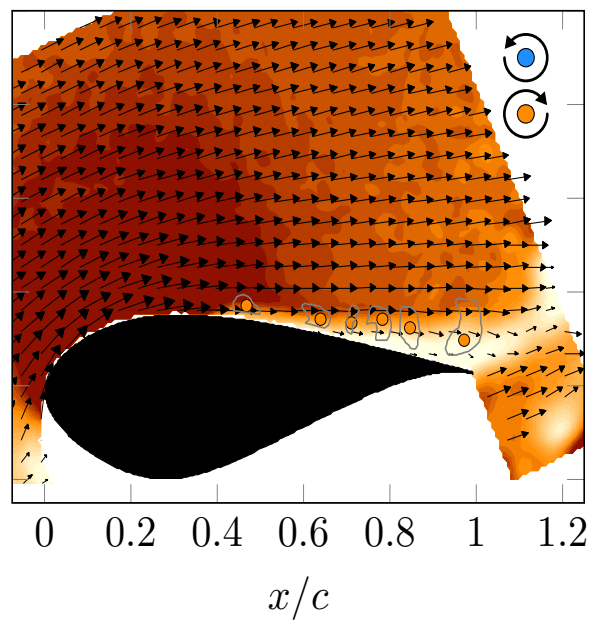

(b) $\alpha=15^{\circ}, \tau=0.138$

Figure 4.5: Instantaneous Velocity at low angles of attack where trailing edge stall develops briefly before flow reattaches after motion begins.

As the flow passes the first third of the chord, it is decelerated due to an adverse chord-wise pressure gradient. In Figure 4.4(b), flow reversal begins to appear near the trailing edge at $x / c=0.9$ and immediately downstream of the separation point at $x / c=0.7$. Beyond time $(b), \beta$ and $\bar{d}_{v}$ increase, indicating that vortices are growing and shifting away from the surface as stall develops.

By further increasing the angle of attack to $\alpha(0.3)=20.4^{\circ}$ at time $(c)$, Figure 4.4(c) shows growth in the stall volume and vortices which emerge closer to the leading edge. Furthermore, significant flow reversal at $x / c=0.85$, coupled with low velocities near the surface allows for induced vortex formation as counter-clockwise vortices appear in proximity to the surface within the separation zone. During this time, the presence of the primary stall vortex delays full separation. The devel- 
opment of the primary stall vortex is observed in the growth of $d_{v}$ that precedes an increase in the separation angle. This phenomena continues until the angle of attack is sufficiently large such that the primary stall vortex is shed. The shedding of the primary stall vortex triggers the fully stalled stage, as shown in Figure 4.4(d). At this angle of attack, the stalled volume of air reaches its maximum size with relatively small variations as $\beta$ and $\bar{d}_{v}$ fluctuate around their largest magnitudes. This stage is characterized by a large velocity deficit which exists over nearly the entire chord. Over the majority of the blade surface, there is significant vortex-tovortex interaction in a large recirculation zone. At time $(e)$, the angle of attack has decreased to $\alpha(0.7)=20.5^{\circ}$, at this point $\beta$ and $\bar{d}_{v}$ appear to decline slightly and vortices recede towards the blade surface, as seen in Figure 4.4(e). The final stage of flow reattachment is signified by a rapid drop in $\beta$ and $\bar{d}_{v}$ at $\tau \approx 0.77$. Physically, this process appears as a periodic growth and shedding of vortex build up over $60 \%$ of the chord as flow works to reattach. The irregular vorticity build up stops as flow reattaches at angle of attack of $\alpha(0.77)=17.3$ and remains attached through time $(f)$, seen in Figure 4.4(f).

\subsubsection{Three-dimensional stall characteristics}

To capture the three-dimensional effects present during the stall cycle, the out-ofplane velocity gradient, $\partial w / \partial z$, is obtained via the continuity equation. Results are presented in Figure 4.6 for the angles of attack shown in Figure 4.4. The gradient is normalized by $c / U_{\infty}$ and a positive magnitude (blue) indicates radial flow accelerating from the root towards the tip.

At low angles of attack, shown in Figure 4.6(a), the flow over the surface of 


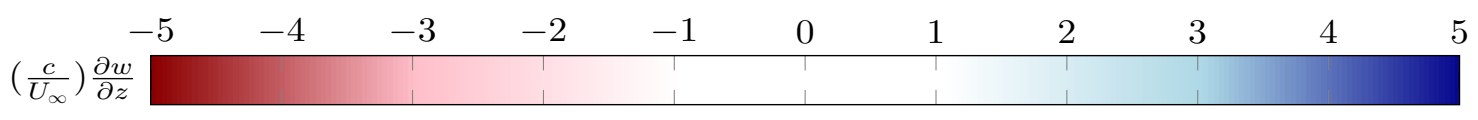

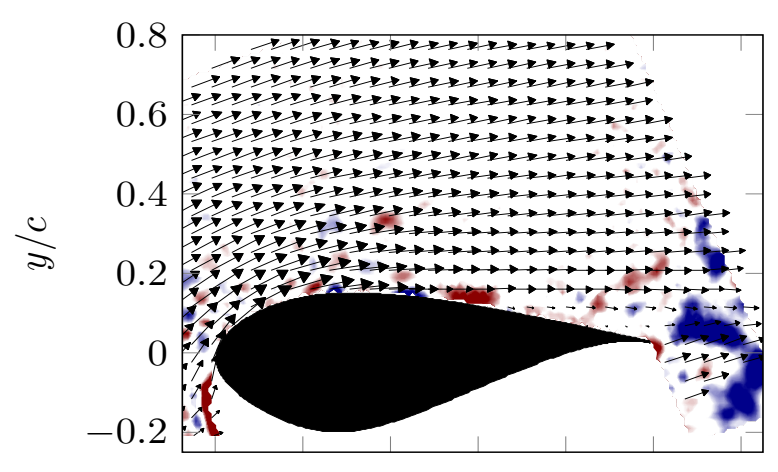

(a) Attached Flow, $\alpha(\tau)=12^{\circ}$

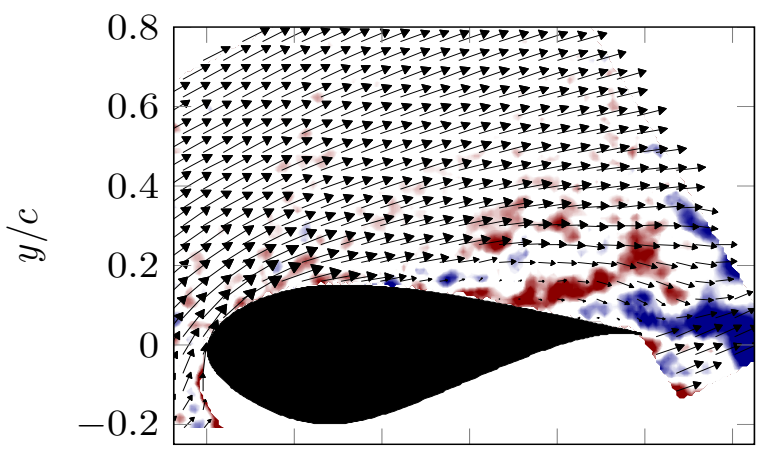

(c) Stall Development, $\alpha(\tau)=20.4^{\circ}$

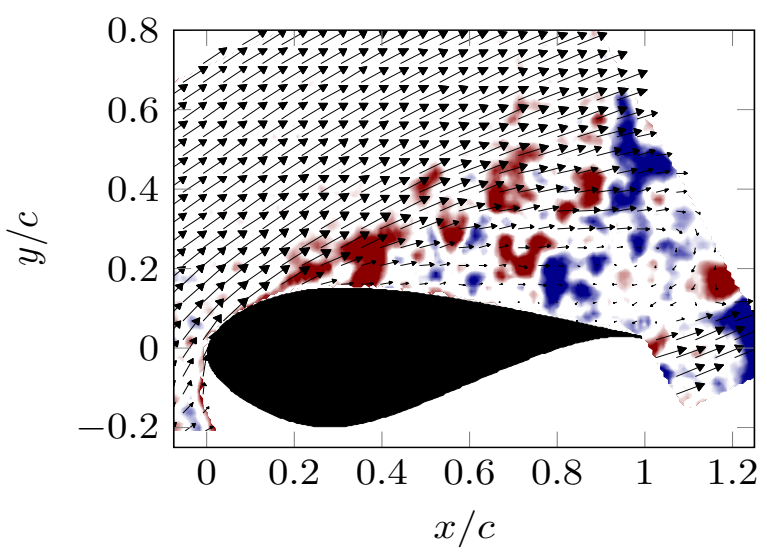

(e) Reattachment, $\alpha(\tau)=20.5^{\circ}$

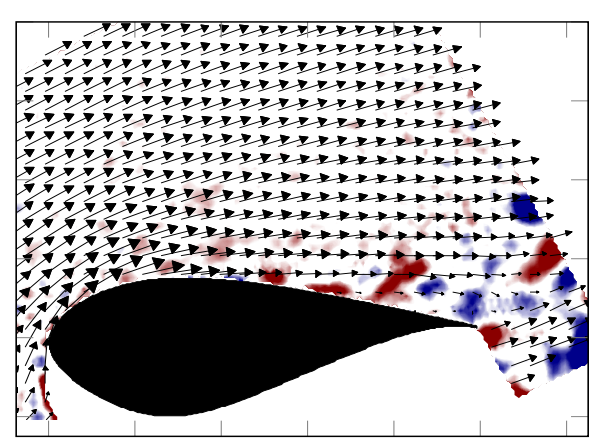

(b) Stall Initialization, $\alpha(\tau)=19.6^{\circ}$

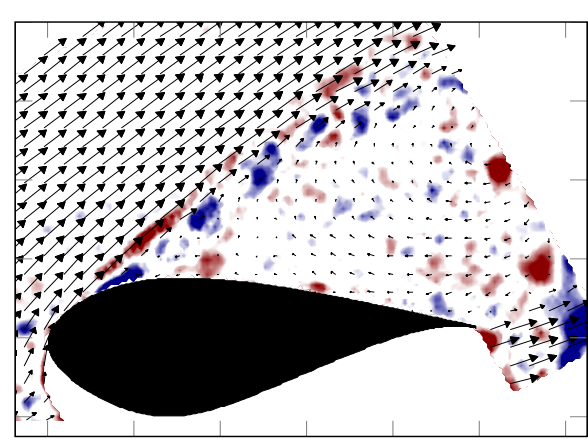

(d) Full Stall, $\alpha(\tau)=24^{\circ}$

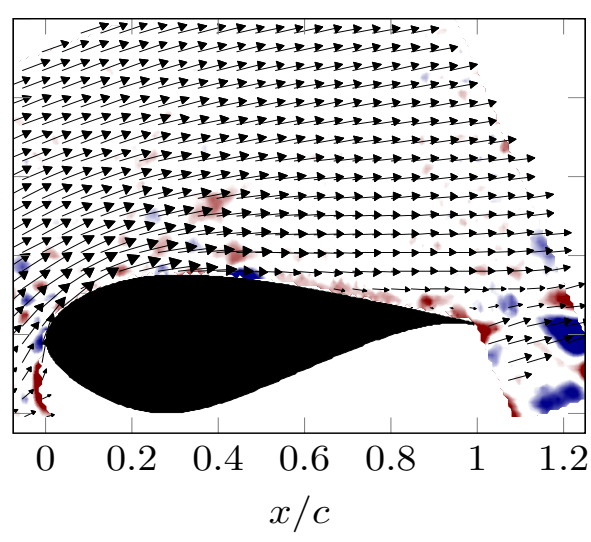

(f) Reattached Flow, $\alpha(\tau)=12^{\circ}$

Figure 4.6: Averaged continuity based calculation of $\partial w / \partial z$ for a pitching cycle of $12^{\circ}-24^{\circ}-12^{\circ}$, plotted with instantaneous velocity vectors. 
the blade lacks a distinct gradient in either direction and is radially homogeneous. However, once the flow passes over the trailing edge, three-dimensional effects are present in the wake. As the blade pitch increases and trailing edge stall begins to e, in Figure 4.6(b), out-of-plane effects are manifested specifically at the shear layer, beginning from $x / c=0.8$. At the point of separation, there is a positive peak in the gradient, showing that flow is accelerated towards the tip. Accompanying the positive radial gradient, a negative peak appears directly upstream the separation point at $x / c=0.75$.

As the angle of attack increases further and the separated volume grows, the number of peak values, both positive and negative, increases; revealing the importance of three-dimensionality during stall development. In Figure 4.6(c), just upstream of the point of separation at $x / c=0.7$, a negative gradient appears. Inside of the stalled volume, a positive spanwise velocity gradient is observed near the top of the shear layer and near to the surface of the trailing edge. When the blade pitch is at its maximum, Figure 4.6(d), the peaks follow the shear layer away from the surface of the airfoil. The peaks remain near the outer portion of the stalled volume, while inside of the stalled region, prior to the trailing edge, radial velocity gradients are significantly diminished. During the process of reattachment, as the shear layer retreats towards the surface, areas of positive and negative velocity gradients enter the recirculation zone previously present during full stall, as shown in Figure 4.6(e). Once reattached, the flow is two dimensional again, as shown in Figure 4.6(f). Evaluation of continuity leads to the conclusion that the shear layer over the blade surface is highly three-dimensional, particularly near the separation point. Additionally, this provides evidence that as the shear layer is formed during 
separation or is breaking down during reattachment the spanwise velocity gradients are playing a role in the aerodynamic forces over the blade.

\subsubsection{Proper orthogonal decomposition}

The POD of the associated two dimensional velocity field, $\tilde{u}_{i}(x, y)$, is defined by the spatial modes $\psi_{i}$ and their relative energy contribution at a given moment in time, indicated by the time development coefficient, $a_{i}(\tau)$, from Equation 2.13. In Figure 4.7, contour maps and vector plots of the first four spatial POD modes are plotted over the blade cross-section with normalized $x / c$ and $y / c$ coordinates. Herein, the shape of the energetic structures gives a qualitative representation of the flow states associated to the specific mode.

Figure 4.7(a) shows the first spatial POD mode, $\psi_{1}$, which captures the dominant mode within the system during the pitching cycle. This energy distribution shows a maximum near the leading edge and a minimum appearing at $x / c=0.8$, which remains close to the surface of the blade and extends away from the trailing edge.

The second mode, shown in Figure 4.7(b), has its maximum concentrated over the chord of the cross-section, extending away from the surface. The maximum is bounded by two minima, one extending from the leading edge and the other extending from the trailing edge. These two minimum lines appear at the same locations as the shear layers from Figure 4.4(d), which surround a stall cell at the highest angle of attack.

The third spatial mode, $\psi_{3}$, plotted in Figure 4.7(c) has two maxima and three minima. The largest maximum extends away from the surface at the leading edge and the second emerges from approximately the mid-chord, $x / c=0.55$, and remains 


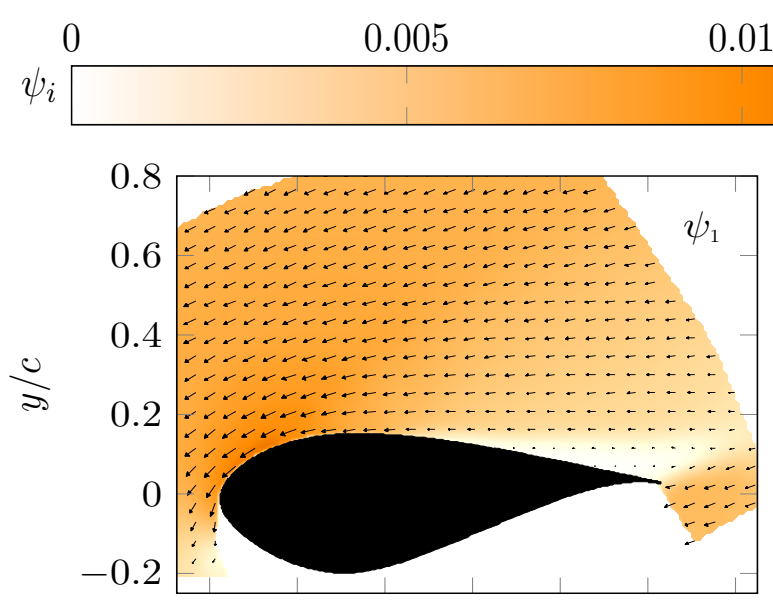

(a) $1^{\text {st }}$ POD mode $\left(\psi_{1}\right)$

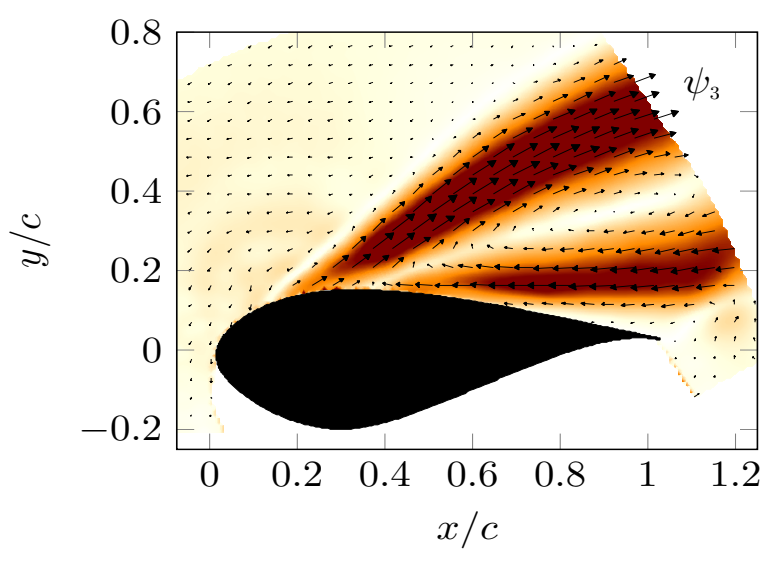

(c) $3^{\text {rd }}$ POD mode $\left(\psi_{3}\right)$

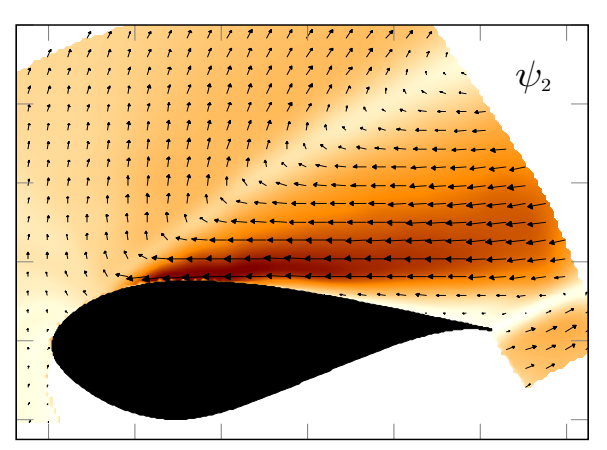

(b) $2^{\text {nd }}$ POD mode $\left(\psi_{2}\right)$

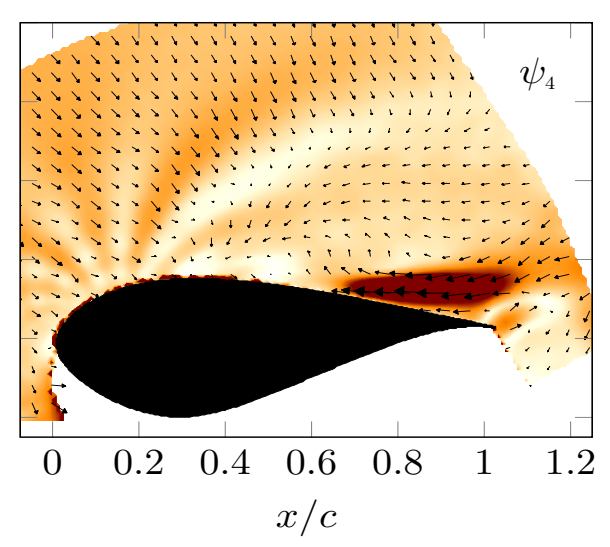

(d) $4^{\text {th }}$ POD mode $\left(\psi_{4}\right)$

Figure 4.7: Contour plots of the first four spatial POD modes $\psi_{1}, \psi_{2}, \psi_{3}$ and $\psi_{4}$ associated with the experimental motion profile $\alpha(\tau)=12^{\circ} \rightarrow 24^{\circ} \rightarrow 12^{\circ}$

near the surface until the trailing edge. The smaller maximum appears to occupy a similar area to the minimum from Figure 4.7(a), while the largest maximum follows the same path as the minimum that extends from the leading edge in Figure 4.7(b). These areas appear in the velocity contours as areas of high shear, where high velocity gradients are present during the pitching cycle. The fourth spatial POD mode, shown in Figure 4.7(d), has one distinct maxima located near the trailing 
edge in the same region as the minimum from Figure 4.7(a).

\subsubsection{POD time coefficients}

To illustrate the influence of each spatial mode as the blade is pitched, the associated time coefficients, $a_{i}(\tau)$, are plotted with the separation point, $f(x / c)$, in Figure 4.8. The separation point, $f$, is approximated by taking the $(x / c)$ location where negative streamwise velocity measurements occur near to the blade surface. All four coefficients and the separation point are plotted on top of the motion profile, $\alpha(\tau)$, shown on the right vertical axis. Magnitudes of the time coefficients are normal-

ized by their associated eigenvalue, $\sqrt{2 \lambda_{i}}$, and the location of the separation point, $f(x / c)$, are plotted on the left vertical axis. Observing the time coefficients individually, the behavior and contribution of each as a function of angle of attack is described. Herein, the time dependent POD coefficients are discussed chronologically in terms of their magnitudesub over the duration of the pitching cycle.

Prior to the beginning of the pitching cycle, the time coefficient associated with the first spatial POD mode, $a_{1}$, in Figure 4.8, is at its largest. During this period, the second POD time coefficient is relatively small, $\left|a_{2}\right|<0.1$ amounting to approximately $7 \%$ of its maximum. Similar to the second coefficient, $a_{3}$ and $a_{4}$ begin this time series at minimum values of $a_{3} \approx 0$ and $a_{4} \approx-0.5$. Moreover, a strong connection between $a_{1}$ and the attached flow stage shown in Figure 4.4(a), while the remaining three coefficients are less significant. During this period the separation point location is shown to fluctuate between $0.65<f(x / c)<1$, an indication of persistent trailing edge stall prior to the pitching cycle.

Once the pitching cycle begins, the coefficients maintain consistent behavior 


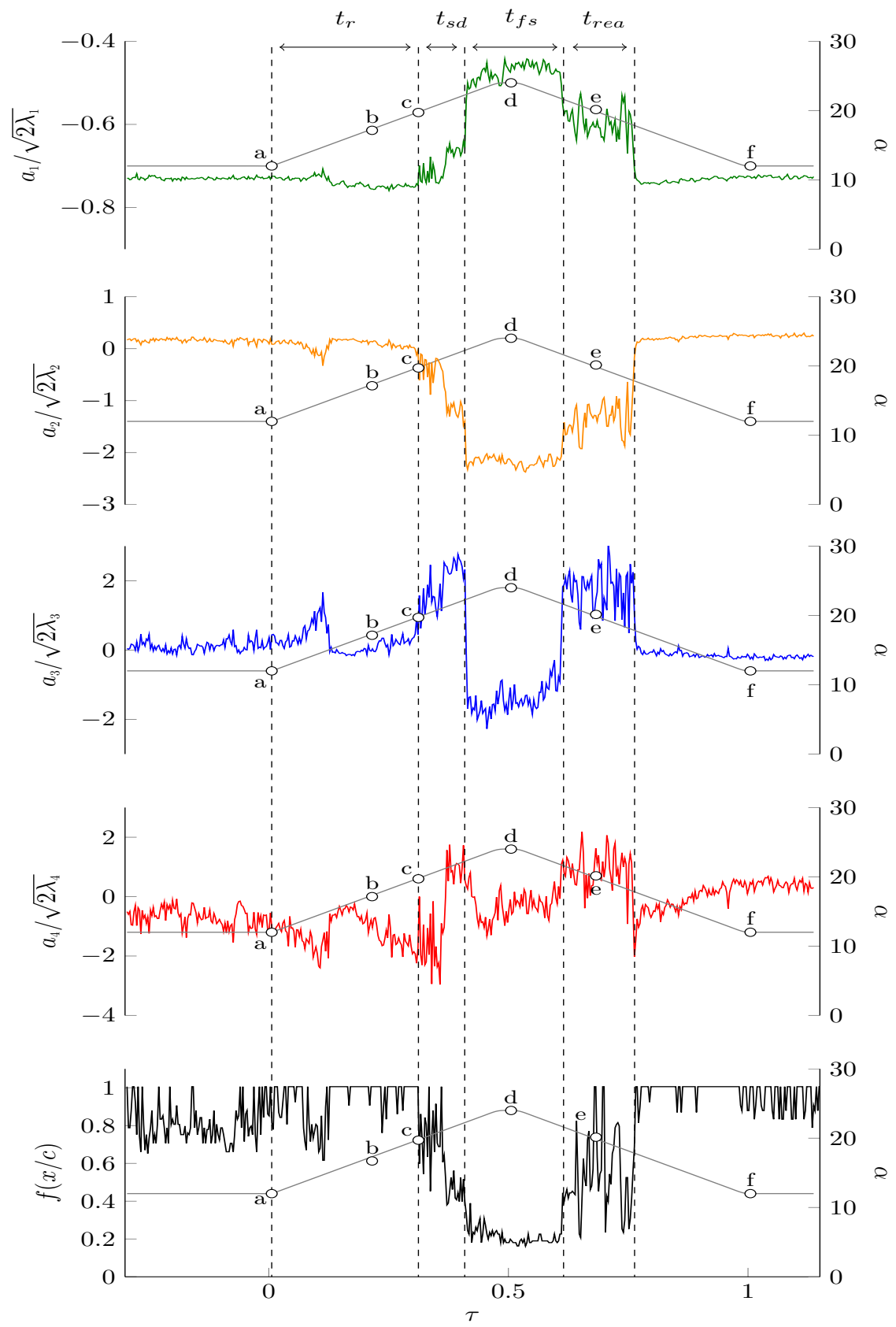

Figure 4.8: Normalized POD time coefficients, $a_{1}, a_{2}, a_{3}, a_{4}$ and separation point $f(x / c)$ plotted against angle of attack as a function of time, $\alpha(\tau)$. 
until the angle of attack reaches $\alpha(0.124)=14.7^{\circ}$, when for a brief instance the trailing edge stall grows, as shown in Figure 6.1(a). In response to the growth of trailing edge stall, the contribution of $a_{1}$ is reduced while the other three coefficients show an increase. During this process, the third and fourth coefficients fluctuate considerably, showing increased sensitivity to the growth and shedding of the stalled volume when compared to $a_{1}$ and $a_{2}$. After the stalled volume is shed and the flow reattaches, shown in Figure 6.1(b), $a_{1}$ and $a_{2}$ return to their attached flow values and $a_{3}$ and $a_{4}$ decrease, but show higher variability in this case than is seen in $a_{1}$ and $a_{2}$.

Beyond this brief instance of stall development, the first two POD coefficients remain near their respective values, previously seen at time $(a)$, until the angle of attack reaches $\alpha(0.3)=19.8^{\circ}$ at time $(c)$ which corresponds to the beginning of stall development, as seen in Figure 4.4(c). Contributions of $a_{3}$ and $a_{4}$ both grow leading into time $(c)$, and are both affected by the initialization of stall. After time $(c)$, the flow begins its transition towards stall, during this process a two step reduction in $a_{1}$ is observed. Simultaneously, $a_{2}$ begins to increase rapidly to $47 \%-60 \%$ of its maximum value, passing through two step-changes as the value increases to its maximum by time $(d)$. Similar to the first two time coefficients, $a_{3}$ and $a_{4}$ show a dramatic change at time $(c)$. However, it is during the transitional period between times $(c)$ and $(d)$, prior to reaching full stall, that $a_{3}$ and $a_{4}$ reach their maximum. At an angle of attack of $\alpha(0.41)=22.4^{\circ}$, the blade section enters a fully stalled state, at which point the magnitude of $a_{1}$ drops to $60 \%$ of its maximum, $a_{2}$ arrives at its maximum, and $a_{3}$ changes sign. During the transition, the separation point location history reflects the step changes shown by the coefficients as it fluctuates 
about $x / c=0.6$ after time $(c)$, shifting to $x / c \approx 0.4$ before reaching its minimum value of $x / c \approx 0.2$ prior to time $(d)$.

At time $(d)$, the first POD coefficient reaches its absolute minimum and the value of $a_{2}$ is at its maximum level where $\alpha(0.5)=24^{\circ}$. At this angle of attack, the blade cross section is experiencing full stall based on the velocity contour in Figure 4.4(d). Based on the relatively high magnitudes present at times $(c)$ and $(d)$, it appears that $a_{3}$ and $a_{4}$ contribute to the flow dynamics during the transitional and fully stalled states. Although during full stall, contributions from $a_{4}$ are reduced compared to values prior to full stall.

At $\alpha(\tau) \approx 21^{\circ}$, shown just before time $(e)$, as the flow begins to reattach, the magnitude of $a_{1}$ rapidly increases back to $80 \%$ of its original value. The contribution of $a_{1}$ remains relatively consistent until there is one final step increase at $\alpha(0.77)=$ $17.7^{\circ}$, where the flow reattaches and $a_{1}$ returns to its maximum. The magnitude of $a_{2}$ remains high until the pitch is reduced beyond $\alpha(0.6)=21^{\circ}$, where the flow begins to transition to reattachment, at which point it starts decreasing to $60 \%$ of its maximum. Once the angle of attack reaches a value of $\alpha(0.77)=17.7^{\circ}$ beyond time $(e)$ and flow reattaches, $a_{2}$ returns to its minimum, $a_{2} \approx 0$, and remains at that value through time $(f)$. The influence of $a_{3}$ is still relatively high at time $(e)$ and remains so until the angle of attack is reduced beyond $\alpha(0.77)=17.7^{\circ}$, at which point the coefficient changes signs once again and returns to approximately $10 \%$ of its maximum. The third time coefficient remains near its minimum of $a_{3} \approx 0$ as the angle of attack returns to $\alpha(1.0)=12^{\circ}$ at time $(f)$, thus reinforcing its independence from the attached flow state. At the moment of reattachment, $a_{4}$ changes sign and reaches a near maximum before slowly tapering down to a relatively small value. 
As the blade pitch is reduced, the separation point retreats from the leading edge towards $x / c \approx 0.4$ an fluctuates between $0.2<f(x / c)<1$ throughout the transition prior to $\alpha(0.77)=17.7^{\circ}$. During the extent of the pitching cycle, behavior of $a_{1}$ and $a_{2}$ tend to oppose each other. At times where the first mode has its maximum magnitude, the second mode is at its minimum and the opposite is also true that when $a_{1}$ is near its smallest magnitude, the $a_{2}$ is near its maximum. When the contributions of these coefficients are compared to the behavior of the separation point, it is concluded that $\psi_{1}$ represents attached flow, $\psi_{2}$ corresponds to the fully stalled state. Due to their relatively large sizes during transitional stages, the third and fourth modes are considered contributors to the transitional stages of stall development and reattachment.

\subsection{POD based time scales}

The previous analysis has shown that one advantage of using POD on time resolved data is that the associated time coefficients, $a_{i}(\tau)$, reveal a strong correlation to the various stages of dynamic stall. Following the behavior of the coefficients, several distinct regimes are noted for each POD time coefficient during the pitching cycle, shown in Figure 4.8. These regimes have been highlighted as time intervals that describe the duration of each stage of the stall cycle. The first time scale that is observed is termed the reaction time, $t_{r}$. This time interval begins at the moment of initial motion of the blade and end is marked by a dramatic change in behavior of the POD time coefficients. The second time interval that is identified is the time the blade experiences the onset of stall prior to reaching a fully stalled state. This time interval is termed the stall development time and is represented by $t_{s d}$. The 
Table 4.1: Dynamic stall time scales identified during full pitching cycle, convection time $\left(t U_{\infty} / c\right)$, duration as portion of $\tau$, and range of angles $\Delta \alpha\left(^{o}\right)$.

\begin{tabular}{||c|c|c|c||}
\hline Time scale & Convection Time $\left(t U_{\infty} / c\right)$ & $\Delta t_{i} / t_{m}$ & $\Delta \alpha\left(^{o}\right)$ \\
\hline Reaction, $t_{r}$ & 152.4 & 0.348 & $12-19.3$ \\
\hline Stall development, $t_{s d}$ & 40.5 & 0.093 & $19.3-22.3$ \\
\hline Full stall, $t_{f s}$ & 77.3 & 0.177 & $22.3-24-21.7$ \\
\hline Reattachment, $t_{r e a}$ & 63.3 & 0.145 & $21.7-17.3$ \\
\hline
\end{tabular}

beginning of this stage is marked by the emergence of stall development and a change in the POD coefficients is observed and ends when the primary stall vortex is shed, corresponding to a second dramatic change in the time coefficients. The change marks the beginning of the next interval, identified as the period of time that the blade is experiencing full stall, $t_{f s}$. The phase ends when angle attack is reduced to $21.7^{\circ}$ and the flow begins to reattach, during this process the coefficients step back towards their previous levels found during stall development, $t_{s d}$. The fourth and final time interval is the time required for the flow to reattach to the surface and is termed the reattachment time scale $t_{r e a}$. This time interval begins as the angle of attack drops below $21.7^{\circ}$ and ends when the flow reattaches at $\alpha(\tau)=17.3^{\circ}$. Values for each time scale are presented as function of convection time $\left(t U_{\infty} / c\right)$, percentage of motion $\Delta t_{i} / t_{m}$, and range of angle, $\Delta \alpha(\tau)$ in Table 4.1.

The reaction time, $t_{r}$, is the longest time scale identified and is nearly twice the next largest time scale, $t_{f s}$. Stall begins to develop, at $\alpha(\tau)=19.3^{\circ}$ marking the beginning of $t_{s d}$ and reaches full stall at $\alpha(\tau)=22.3^{\circ}$, which is the shortest of the estimated time scales. The period of time spent in full stall, $t_{f s}$ is approximately $17.7 \%$ of the pitching motion. By identifying this time scale, it is seen that the angles 
of attack for which full stall begins and ends differ by $0.6^{\circ}$. During recovery from a fully stalled state, reattachment is not imminent due to the unstable nature of stall. Consequently, the required angle of attack for reattachment, $\alpha(\tau)=17.7^{\circ}$, is lower than for separation, $\alpha(\tau)=19.3^{\circ}$. Moreover, the time interval, $t_{\text {rea }}$, associated with the transition from stall to reattachment is 1.5 times greater than the stall development time, $t_{s d}$, thus indicating that flow separation is delayed by the primary stall vortex. In the case of reattachment, the process occurs more slowly and well below the angle of attack at which separation occurred; this is taken as an indication of hysteresis characteristic of pitching airfoils. Based on the difference in the angle of attack values at which flow moves from one state to the other, both attached flow and stall demonstrate a resistance to state change. When the flow is attached, there is an apparent resistance to stall developing. Once stall is onset, the transition to a full stall takes only a few degrees change in the angle of attack.

\subsubsection{POD reconstruction}

Based on the previous results, the information provided by the first four POD modes which represents $99 \%$ of the energy. To demonstrate the ability of the first four POD modes to capture the primary flow structures, modal reconstructions are found for three of the stall stages, attached flow, stall development and fully stalled flow. Figure 4.9 (a), (c), (e) show the POD reconstructions for the velocity fields at angles of attack related to attached flow, stall development and fully stalled stages. These are directly compared with the corresponding normalized instantaneous velocity fields, $\tilde{u}_{i} / U_{\infty}$, Figure 4.9 .

The POD reconstruction, using the first four modes, provides information about 


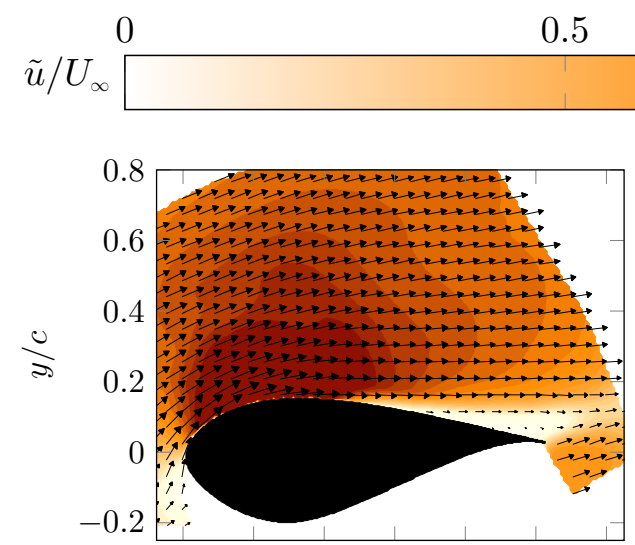

(a) POD reconstruction of velocity field at $\alpha(\tau)=12^{\circ}$

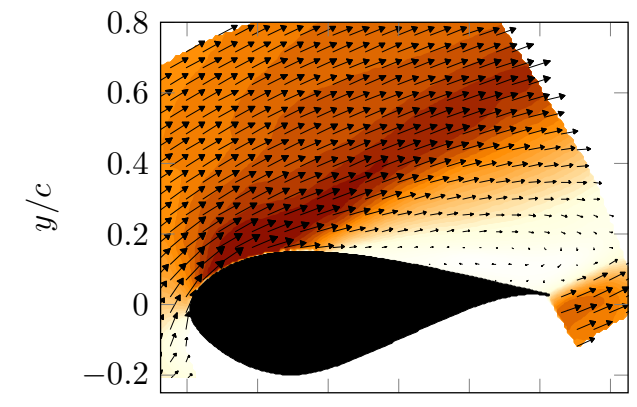

(c) POD reconstruction of velocity field at $\alpha(\tau)=20.4^{\circ}$

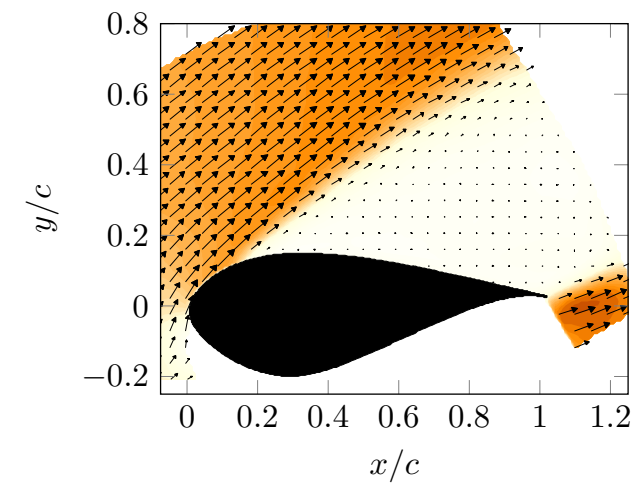

(e) POD reconstruction of velocity field at $\alpha(\tau)=24^{\circ}$
1

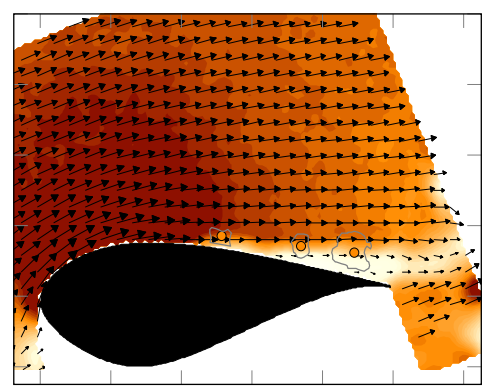

(b) Instantaneous Velocity, $\tilde{u}_{i}$, at $\alpha(\tau)=12^{\circ}$

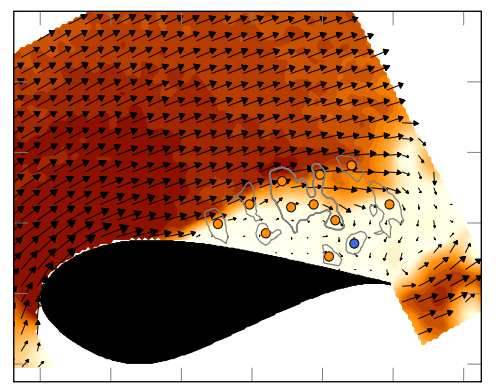

(d) Instantaneous Velocity, $\tilde{u}_{i}$, at $\alpha(\tau)=20.4^{\circ}$

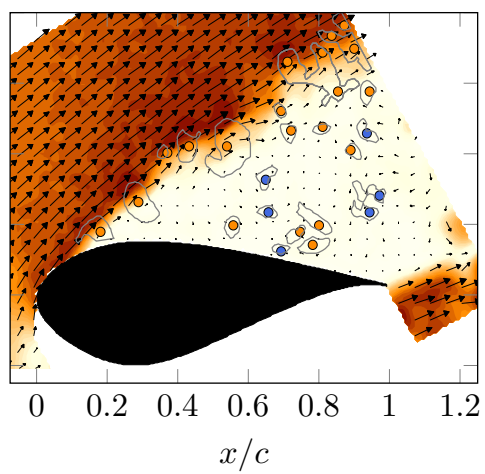

(f) Instantaneous Velocity, $\tilde{u}_{i}$, at $\alpha(\tau)=24^{o}$

Figure 4.9: Reconstruction of the velocity field using the first four POD modes plotted adjacent to the corresponding velocity fields during attached flow, stall development, and fully stalled stages. 
the location of the separation point, the angle of the shear layer, and the volume of stalled air contained within the stall envelope. In the reconstructions of attached flow and full stall, the thickness of the wake and location of the shear layer are visibly similar to the instantaneous field. The distinct shapes of the instantaneous flow field are lost due to the smoothing affect of using just the first four POD modes, most noticeably in Figures 4.9(d) and 4.9(f). The reconstruction of the stall development stage does not capture the instantaneous flow as accurately as the fully stalled and attached flow reconstructions, indication of the lack of small scale information provided by the first four POD modes.

To illuminate the contribution of each of the POD modes to the overall flow structure as a function of angle of attack and time, the instantaneous velocity fields from Figure 4.2 are reconstructed in Figure 4.10 using a combination of two modes, $\psi_{1-2}$, three modes, $\psi_{1-3}$, and all four modes, $\psi_{1-4}$. The velocity fields from time $(f)$ are omitted due to their behavioral resemblance to time $(a)$. Figure 4.10 has five velocity fields reconstructed oriented by row, each column corresponds to the number of modes, $\psi_{n}$, used in the reconstruction. To aid the discussion, boxes are placed around the images that change based on the number of modes that contribute to the overall shape of the flow for each stage, where additional features are not observed in subsequent mode inclusion. Evaluating the figures by row, the attached flow shows no change as the number of modes included is increased beyond the first two modes. These results serve to reinforce conclusions about the influence of the first POD time coefficient being the dominant mode during attached flow. Images corresponding to stall development shown in the second row are improved with each additional mode included in the reconstruction. The contribution of the third modes adds to 
the definition of the flow field and additional size to the separated area, while the the fourth POD mode acts as a subtraction to reduce the area of the stall envelope toward a shape of the velocity field shown in the stall initialization stage identified in Figure 4.4(b). Once stall development has begun at time $(c)$, shown in the third row, the flow structure is captured by the first three modes. The first two modes create the basic shape present, and the third mode provides the description of the velocity distribution within the stalled area. The fourth mode does not appear to add meaningful information to the overall flow structure for this phase. In row four, the flow is fully stalled over the blade cross-section. At this stage, the majority of information contributing to the reconstruction is provided in the first two modes. The only clear contribution from the third mode is seen in the shear layer that extends from the leading edge, where the velocity gradient becomes more clearly defined. The contribution for the fourth mode is negligible. The reattachment phase shown in row five has nearly the same contributions as the stall development phase, where the first two modes provide the basic shape of the stall envelope and the third mode provides a detailed velocity distribution within the separated area.

In summary, the POD reconstructions show that during attached flow, the average flow structure is captured with only two POD modes, during transition the complex flow requires all four modes, and during full stall, only three POD modes are required for an accurate reconstruction of the general flow characteristics.

\subsection{Conclusions}

Dynamic stall cycle of an experimental three-dimensional wind turbine blade was investigated with time resolved PIV. Velocity fields provided by the PIV were used 

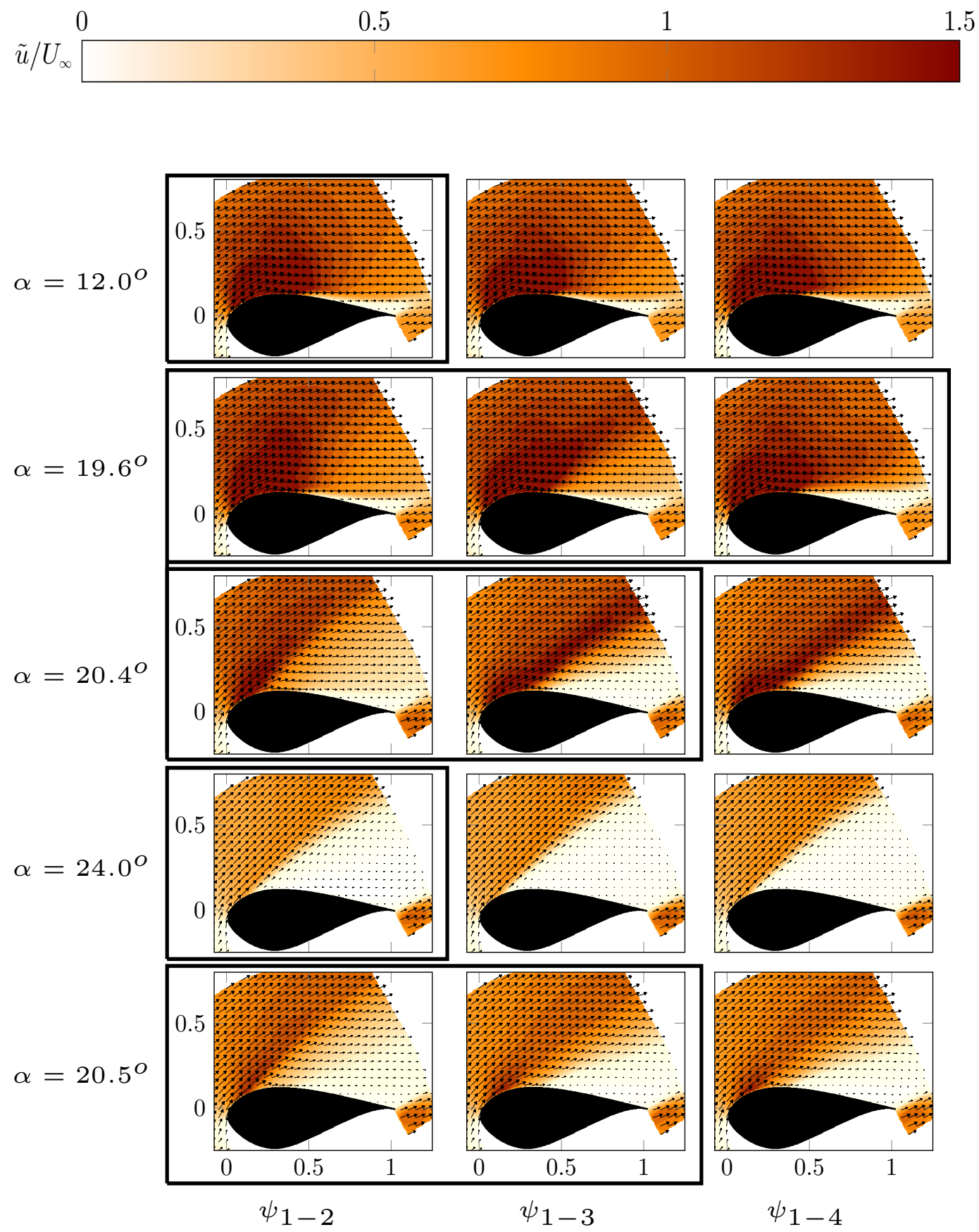

Figure 4.10: Reconstruction with 2, 3, and 4 modes (columns) of the velocity field using the first four POD modes plotted with the corresponding velocity fields during attached flow, stall development, fully stalled, and reattachment stages (rows). 
to identify stall stages based on the formation and shedding of vortices based on the $\Gamma_{2}-$ and $\lambda_{2}-$ criterion. Using these criteria to determine the size and angle of vortex detachment, it was seen that during a pitching cycle, the growth of vortices over the airfoil surface slightly precedes the displacement of the vortices from the surface. Three-dimensional flow behavior was identified via the continuity equation which provided the out-of-plane velocity gradient. It was shown that the spanwise velocity gradient had its largest influence during stall development and reattachment and that out-of-plane effects are primarily contained within the shear layer. Moreover, results showed that three dimensional effects are ordered in the shear layer, such that negative gradients were consistently found upstream of their positive counterparts. Proper orthogonal decomposition was used to identify the most energetic flow structures present during the stall cycle. The first four modes were found to contain $99 \%$ of the energy. To determine the role of each mode during the pitching cycle, POD time coefficients were compared to the associated velocity fields. The first two POD modes captured the attached and separated flow states, respectively, and the third and fourth combined described stall development and reattachment.

The advantage of using POD on the time resolved data is the relationship between time coefficients, $a_{i}(\tau)$, and dynamic stall stages. Defined by significant changes in the behavior of the POD time coefficients, time scales such as the reaction, stall development, full stall and reattachment times involved in the process were identified. Results revealed that the time required for the reattachment process is $50 \%$ larger and was completed at a lower angles of attack than the process of separation. Within this framework, the time scales associated to stall development and reattachment stages showed a clear resistance to state change. These relationships 
were investigated further, by reconstructing the flow fields using a limited number of POD modes. Results showed that for attached and fully stalled flow, only the first two POD modes were required to reconstruct the primary flow structure. While reconstructions for stall development and reattachment revealed that the third and fourth modes were important, due to the complexity of flow behavior during these processes. It was also noted that the reconstructions during the transitional stages would require a larger number of modes to capture the small scale flow behavior. 


\section{Coherent structure interaction during unsteady separation}

In describing stall delay, three main regions of interest are: the boundary layer, the shear layer, and the stalled region. The physical process of transition from attached flow to separated flow is characterized by Kelvin-Helmholtz stability theory. Separation occurs when the thickness of the boundary layer in front of the separation point is small compared to the dimension of the separated region perpendicular to the main flow direction [75]. As the effective angle of attack increases, a chordwise adverse pressure gradient grows downstream of the blades maximum thickness, allowing vorticity within the boundary layer to accumulate. Vorticity build up obstructs flow along the blade surface, deflecting the free stream away from the surface and resulting in a downstream velocity deficit. Excessive vorticity leads to the formation of vortices which form and are convected over the blade surface causing concentrated pressure fluctuations. The pressure fluctuations act as perturbations in the shear layer, thus playing a significant role in aerodynamic loading. The growth of the perturbations leads to inflections of the shear layer near to the separation point and abrupt movements of the separation point itself [26].

The location of the separation point as a function of angle of attack is obtained through time resolved particle image velocimetry mueasurements. Instantaneous ve- 
locity fields during the stages of the stall cycle are presented following similar methods to those presented in [76]. Through the aid of vortex identification techniques outlined in Section 2, the pattern of vortex shedding and the development of the shear layer are examined as a function of angle of attack. The characteristic amplitudes and frequencies during transition are determined through a multi-dimensional ensemble empirical mode decomposition $(\overline{M E E M D})$ in Section 2. The behavior of the shear layer prior to and during transition, is visualized via Lagrangian coherent structures (LCS) defined by [71] in Chapter (2). By evaluating the locations of the attracting and repelling LCS during stall development in comparison with the resulting $\overline{M E E M D}$ surfaces, the physicality of the $\overline{M E E M D}$ modes is reinforced.

For LCS to accurately represent the material behavior within PIV measurement, the selection of an accurate velocity scale and integration time is crucial. Parameterization of the flow map and corresponding Cauchy-Green strain tensor requires the selection of a velocity scale and integration time to capture the variations in particle trajectories. For stationary flows, this characteristic velocity is taken as a small scale fluctuating velocity within the flow, and the integration time is treated as a constant defined by static geometric characteristics of the flow field. Under unsteady conditions, the fluctuating velocity scales and integration time vary significantly as stall develops. To account for the dynamic variance in these two characteristic scales, the results from the first EMD mode and the separation point, $f$, are used to define the fluctuating velocity scale and integral time scale. The characteristic velocity fluctuations for each sample is extracted by taking the RMS value of the first EMD mode, $\mathbf{u}_{\mathbf{i}}{ }^{\prime}=\sqrt{{\overline{m I M F_{1}}}^{2}}$. The integral length scale which governs the flow within unsteady aerodynamics is taken as the length of the shear layer extending from 
the separation point over the remainder of the chord, defined here as $L=(1-f)$. When $L$ is divided by the fluctuating velocity, $\mathbf{u}_{\mathbf{i}}{ }^{\prime}$, the result is the integration time, $T=(1-f) * c / \mathbf{u}_{\mathbf{i}}{ }^{\prime}$, which determines the number of time steps used to calculate the LCS. For example, during separation the characteristic fluctuation velocity is determined to be $\mathbf{u}_{\mathbf{i}}{ }^{\prime}=1.4 \mathrm{~m} / \mathrm{s}$ and the integral spatial scale is $0.05 \mathrm{~m}$ or $x / c=0.33$ thus yielding an integration time of $0.035 \mathrm{~s}$. Following this method, the fluctuation velocity ranges from as high as $2.65 \mathrm{~m} / \mathrm{s}$ during attached flow, to a low of $0.62 \mathrm{~m} / \mathrm{s}$ during a fully stalled state.

Transitional stages within a stall cycle are selected to examine the LCS interaction during separation. The separation point $f$, which is determined by locating the first occurrence of a negative stream-wise velocity near the airfoil surface, is plotted as a function of time in figure 5.1(a). In figure 5.1(a), the separation point is plotted (black) with the angle of attack (grey) for the $y$-axis and both are plotted as a function of normalized convective time $\tau=t U_{\infty} / c$. The intervals that capture stall development and reattachment are highlighted in gray in figure 5.1(a). PIV snapshots during the transitional stages of stall development and reattachment are selected here for specific analysis using LCS and $\overline{M E E M D}$. To show the dynamic character of the data set, velocity fields for three pitch angles are shown in figure 5.1 revealing the flow behavior for attached 5.1(b), stall development 5.1(c) and fully stalled states $5.1(\mathrm{~d})$.

The regular pattern of vortices being shed during attached flow 5.1(b) and stalled flow 5.1(c) are particularly noteworthy. The unsteady flow behavior captured in figure 5.1(c) is an example of the unsteady nature of the shear layer when flow transitions from attached to fully separated. Unlike the other two velocity fields, 


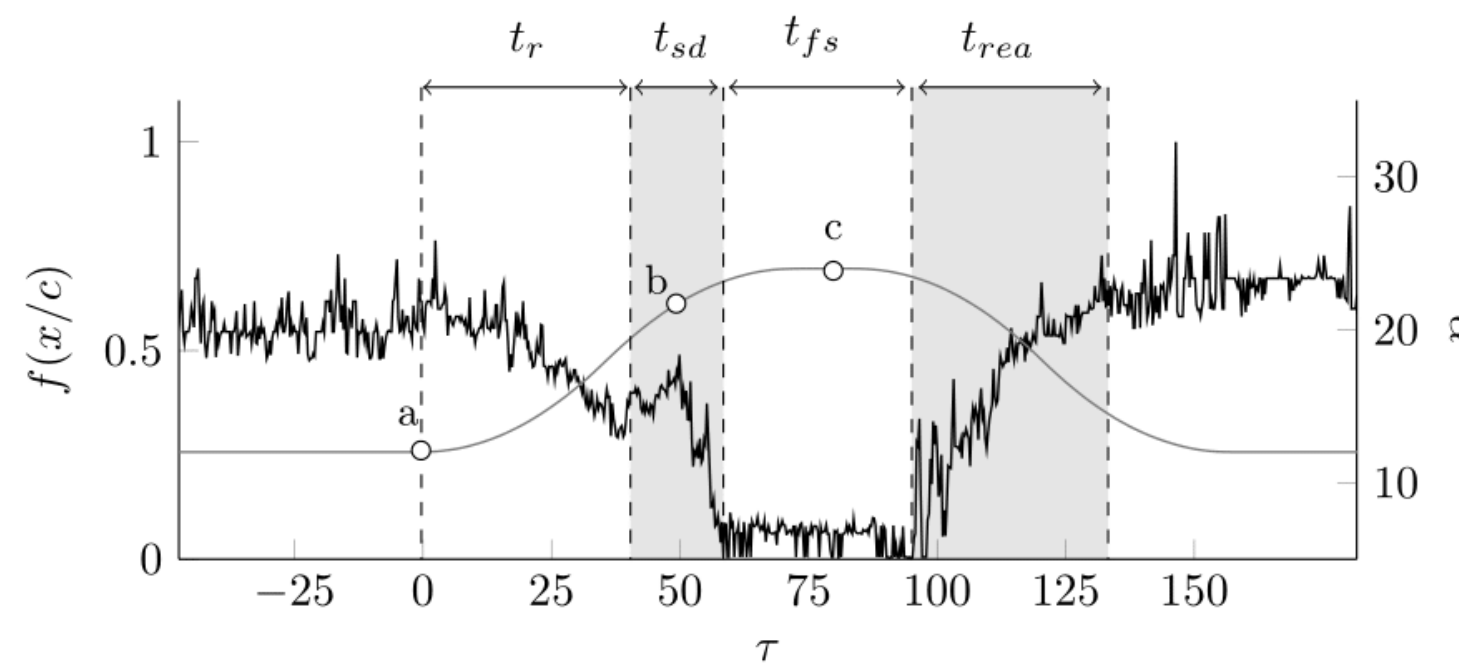

(a) Dynamic Separation point (black) and angle (gray) of attack as a function of normalized time during a full stall cycle.

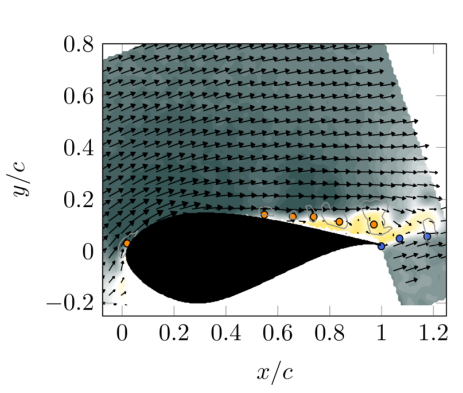

(b) $\quad \alpha=12^{\circ}, \tau=0$

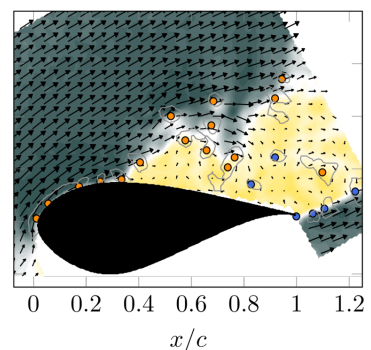

(c) $\alpha=22.2^{\circ}, \tau=52.53$

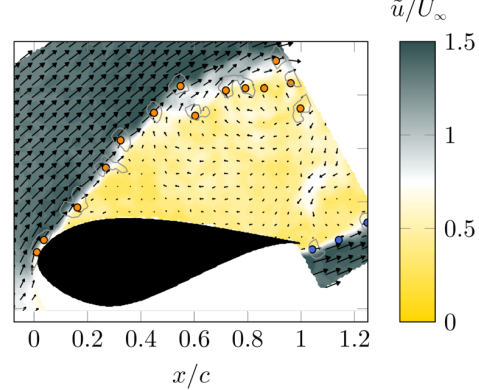

(d) $\alpha=24^{\circ}, \tau=74.93$

Figure 5.1: Instantaneous velocity field at three angles of attack and times that demonstrate the dynamic nature of stall development.

where the shear layer vortices are distributed along the shear layer at constant intervals, the shear layer vortices during transition are redistributed irregularly within the wake. Grouping of vortices corresponds to a large amplitude and low-frequency perturbation of the shear layer. Downstream of the separation point, there is an inflection of the shear layer as the free stream is deflected over the vortex group at $x / c=0.6$. During this period, the separation point changes rapidly during the final 
portion of transition into a fully stalled state. In the most extreme cases during transition, the separation point is observed to move $\Delta f \geq 30 \%$.

\subsection{Lagrangian Coherent Structures (LCS)}

Results in Section 5, show the behavior of shear layer behavior when the flow transitions into stall. During transition, the location of the separation point changes rapidly and the shear layer rolls up along the surface creating inflections downstream of the separation point. The inflection in the shear layer can be a prelude to large movements in the separation point. , LCS material lines are computed to capture the internal flow physics that contribute to the unsteady behavior of the shear layer and separation point during a transition. Dynamic separation during the period of largest change in the separation point is shown in figure 5.2. For this sequence, the angle of attack ranges from $21.81^{\circ} \leq \alpha \leq 22.07^{\circ}$. During this interval, the separation point, as shown in figure 5.1(a), moves from $x / c=0.36$ to $x / c=0.72$ in $\Delta \tau<1$. Figure 5.2(a) depicts stable trailing edge stall. The external shear layer attractor extends from $x / c=0.3$ to beyond the trailing edge without any large scale perturbations. Near the trailing edge, a second attractor envelops the interior of the stalled region, where attracting and repelling LCS appear to intertwine in a swirling formation. As shown by [77], the intersection of attracting and repelling LCS, also known as hyperbolic LCS, are characteristic of low momentum regions.

In figure 5.2(b) a stall cell has developed over the trailing edge of the airfoil. The shear layer originating at $x / c=0.3$ extends over the remainder of the airfoil and is parallel to the free-stream. Inside of the shear layer, a pair of attractors at $x / c=0.65$ are present that converge along and extend away from the airfoil 
surface. Both attractors are bounded by repelling LCS, also found in the adjacent wake flow. The identified vortices remain near the primary shear layer attractor. Within the shear layer, it is shown that the LCS trajectories follow the rotational sense of nearby vortices which appear as perturbations in the shear layer attractor. Figure 5.2(c) is an example of the external shear layer attractor breaking down as it is convected over the airfoil surface. The attractor is replaced by a repelling LCS at $x / c=0.52$. The breakdown is attributed to the presence of a vortex formed in the previous time step which has remained above the surface of the airfoil. The vortex above the surface creates a small separation bubble, encompassed by an attracting LCS. Flow downstream of the vortex returns to the surface following the attracting LCS. The attractor departs from the surface at $x / c=0.7$, meeting a second surface attractor and forming a secondary separation point.

Figure 5.2(d) shows that the surface vortex and secondary separation point have convected downstream from $x / c=0.45$ to $x / c=0.6$ and from $x / c=0.7$ to $x / c=$ 0.78, respectively. While the change in angle of attack is only a tenth of a degree, the movement of the separation point between figure 5.2(b) and figure 5.2(d) is $\Delta f \approx 40 \%$. There are several attractors converging at $x / c=0.78$. The recirculation, at this instance in time, is significant and thus induces a pair of counter-clockwise rotating vortices. Downstream of the attracting LCS emerging at the separation point, two distinct regions are observed. The first is a group of curved repelling LCS laying parallel to each other without crossing any attracting LCS, and a second near the surface, composed of a mix of parallel attracting and repelling LCS. The vortex, which had instigated a temporary separation bubble, has moved away from the airfoil surface in figure 5.2(e). The departure from the surface is accompanied by 
the enveloping LCS and closely bounded by repelling LCS. The secondary separation point has been convected further downstream to $x / c=0.95$ and extends away from the trailing edge.

In figure 5.2(f), the attracting LCS in the shear layer has an inflection just downstream of the separation point. A recirculation zone near the trailing edge is identifiable by the parallel attracting and repelling LCS that form concentric loops. Directly downstream of the separation point at $x / c=0.36$, two repelling LCS follow the adjacent attractor. This pattern is associated with a decreasing flow velocity upstream of the recirculation zone. The obstruction caused by the recirculation eventually leads to a deflection of the shear layer. This aerodynamic reaction occurs as the flow recovers from the vorticity induced stall suppression created by the surface vortex, shown in figures 5.2(c) and 5.2(d). Above the separation region, LCS in the free stream are generally straight and parallel throughout the measurement sequence.

Figure 5.3 presents the LCS behavior during full stall. The interesting features are the separation point, the shear layer and the internal flow structures. For this data series, the angle of attack is constant at $\alpha=24^{\circ}$ over a time span of $85.3<$ $\tau<86.7$. During full stall, the separation point remains at the leading edge of the airfoil. The general behavior of the shear layer during this stage of stall is captured in the attracting LCS. Each figure shows attracting LCS which typically converge at $x / c=0.1-0.2$ and propagate downstream as one material line extending over the entire chord. The presence of a vortex in the shear layer leads to perturbations of the LCS which grow in amplitude as the vortices are convected downstream. Within the stalled region, the LCS exhibit several crossings particularly near the trailing 


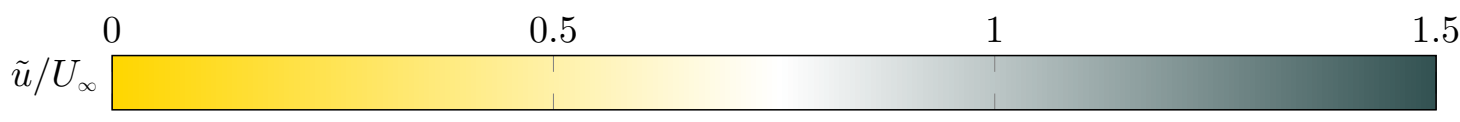

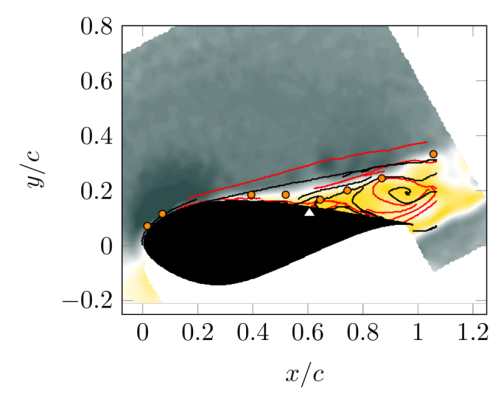

(a) $\alpha=21.81^{\circ}, \tau=50.40$

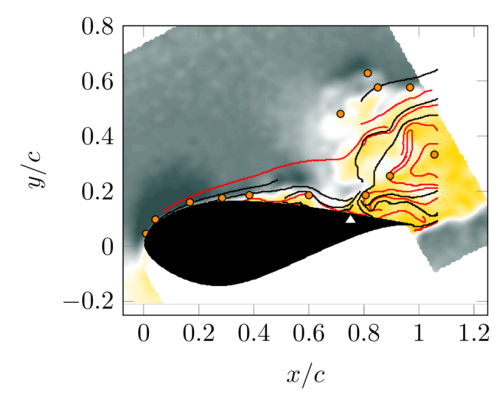

(d) $\alpha=21.96^{\circ}, \tau=51.20$

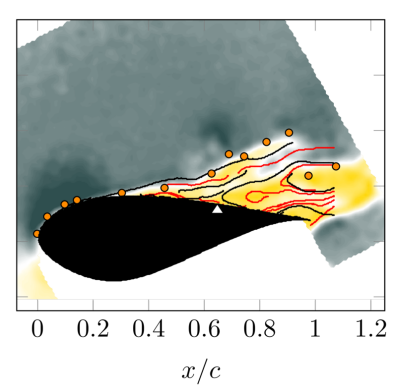

(b) $\alpha=21.86^{\circ}, \tau=50.67$

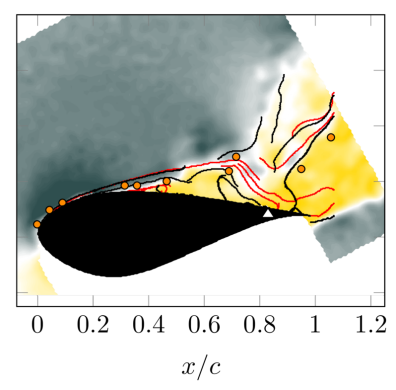

(e) $\alpha=22.02^{\circ}, \tau=51.47$

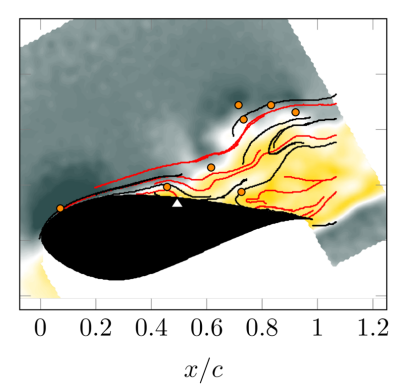

(c) $\alpha=21.91^{\circ}, \tau=50.93$

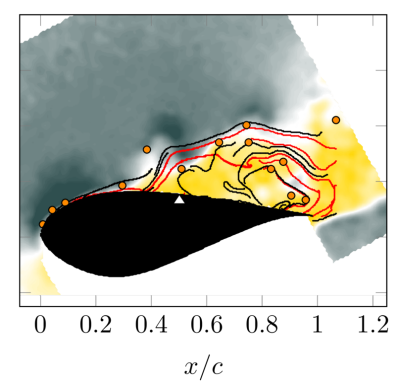

(f) $\alpha=22.07^{\circ}, \tau=51.73$

Figure 5.2: Instantaneous velocity field over six consecutive PIV snapshots during stall development plotted with attracting (black) and repelling LCS (red), separation point $(\triangle)$, and locations of clockwise (orange) and counter-clockwise (blue) rotating vortex core locations.

edge.

In figure 5.3(a), a vortex has formed inside of the LCS just downstream of the leading edge. Attracting material lines converge downstream of this location at $x / c=0.2$. This particular vortex influences the shear layer throughout the time series while convecting downstream at a rate of $\dot{x} \approx 15 \mathrm{~m} / \mathrm{s}$, which is $\approx 0.1 \mathrm{c}$ per image. In each figure, the vortex core is in close proximity to a intertwined LCS, i.e., an area of low momentum, indicating that the vortex itself acts as an obstruction 
to the primary flow within the separated volume. The area of influence increases with each successive time step from less than $10 \%$ of the chord in figure 5.3(a) to $40 \%$ of the chord in figure 5.3(f). Surrounding the repelling LCS, an attracting LCS consistently follows the shape of the inner repelling LCS. The position of the vortex and size of the area of the enveloping LCS are directly related to the surrounding LCS, and the shear layer attracting LCSs. Figure 5.3(e), in particular, demonstrates the influence of shear layer vortices on the neighboring LCS. The frequency of the formation and shedding of vortices is observed in figure 5.3(f). A constant vortex shedding pattern is noted prior to the LCS attracting LCSs converging into the shear layer. Moving away from the surface along the free shear layer, the frequency of vortices residing in the shear layer varies once the internal LCSs intersect with the shear layer. Consequently, the internal recirculation structure has a direct influence on the shear layer perturbation amplitudes, while the vortex shedding frequency at the separation point is held constant.

Figure 5.4 presents LCS results during the reattachment process. Once again, the series follows a vortex which forms at the airfoil surface near the separation point and is convected over the airfoil. Figure 5.4(a) shows the surface vortex forming a separation bubble, which is bound by an attracting LCSs. The point of separation is forced downstream of the surface vortex. An inflection in the shear layer occurs as a result of the forming surface vortex. An internal recirculation zone occupies nearly the entire stalled region and is centered over a pair of vortices. The primary LCS is an attracting LCSs that spirals in a clockwise manner, following the rotational sense of the identified vortex. A repelling LCS lies between the shear layer and the recirculation attracting LCS. 


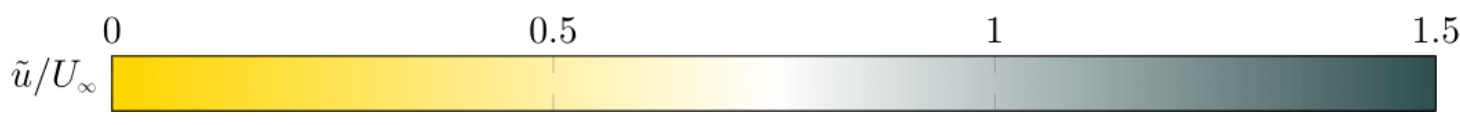

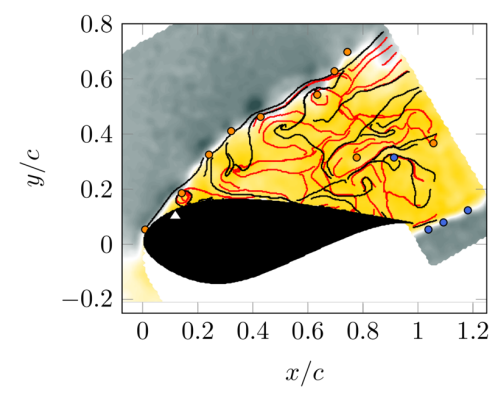

(a) $\alpha=24.0^{\circ}, \tau=85.33$

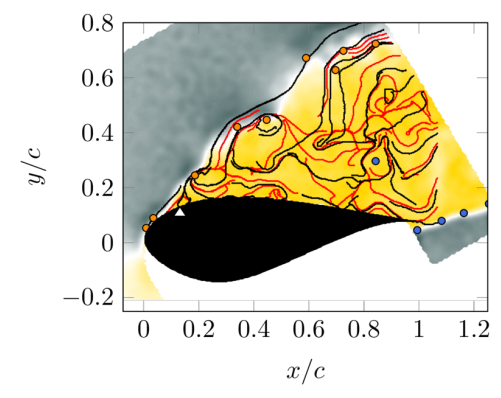

(d) $\alpha=24.0^{\circ}, \tau=86.13$

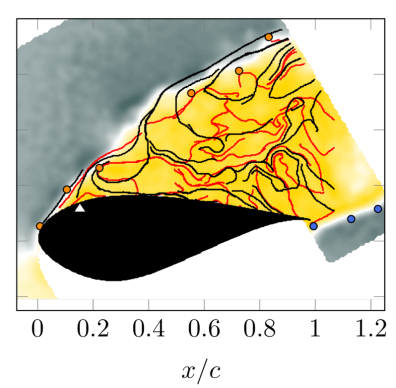

(b) $\alpha=24.0^{\circ}, \tau=85.60$

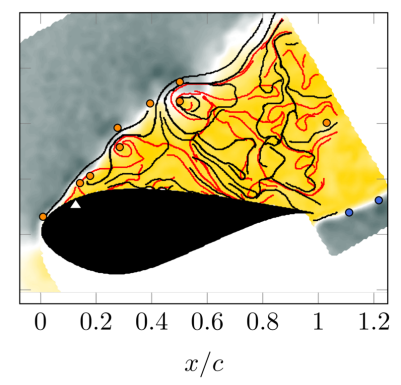

(e) $\alpha=24.0^{\circ}, \tau=86.40$

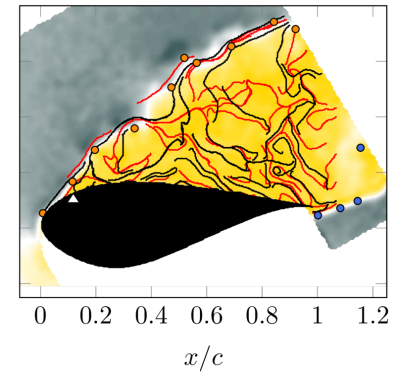

(c) $\alpha=24.0^{\circ}, \tau=85.87$

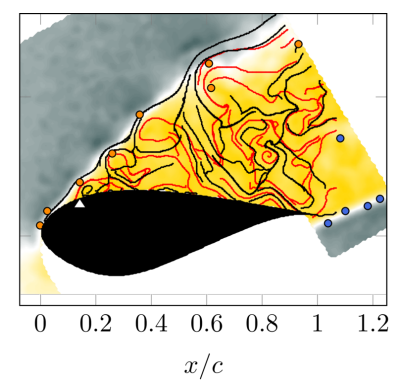

(f) $\alpha=24.0^{\circ}, \tau=86.67$

Figure 5.3: Instantaneous velocity fields over six consecutive PIV snapshots during full stall, plotted with attracting (black) and repelling LCS (red), separation point $(\Delta)$, and locations of clockwise (orange) and counter-clockwise (blue) rotating vortex core locations.

In figure 5.4(b), the surface vortex has grown in size and continues to create an inflection in the shear layer attracting LCSs. The vortex core has convected downstream by $0.15 c$. The recirculation LCS and internal vortex observed in the previous time step have been stretched between the portion of the separated volume directly downstream of the shear layer inflection and a group of repelling LCS near the trailing edge surface. The internal structure within the separated volume is divided into two separate regions by the attracting LCS that runs parallel to the 
external flow.

Figure 5.4(c) gives an example of the recovery process of the reattachment. The shear layer inflection is absent, the internal attracting LCS still divides the separated volume similar figure 5.4(b). The surface vortex has begun to move away from the surface, without moving downstream. Figures 5.4(d)-5.4(f) represent the recurring nature of the reattachment process. Akin to the LCS shapes and locations presented in figure 5.4(a), a vortex now forms near the surface directly downstream of the separation point. The presence of this vortex is accompanied by another inflection in the primary shear layer LCS. Inside the separation region, the surface vortex from figure 5.4(a) has extended its influence and has formed a LCS recirculation zone. The original surface vortex is wrapped up in attracting LCSs, which are in turn surrounded by repelling material lines. The arrangement of LCS has again created an obstruction to the free stream and moves downstream in the next time step.

\subsubsection{Empirical mode decomposition}

The previous section revealed the presence and shedding of a vortex that directly affects the perturbation frequency and amplitudes of the shear layer. Empirical mode decomposition allows for evaluation of TR-PIV fields by separating spatial signal content. Characteristic spatial scales are identified within an Eulerian context. The EMD results are reinforced and provided further physicality with the aid of the identified LCS on each of the described stages.

Results for the first EMD mode during separation, full stall, and reattachment are presented in figure 5.5. One snapshot from each stall stage is presented to provide stagewise comparisons. Within the EMD results, positive velocity fluctuations 


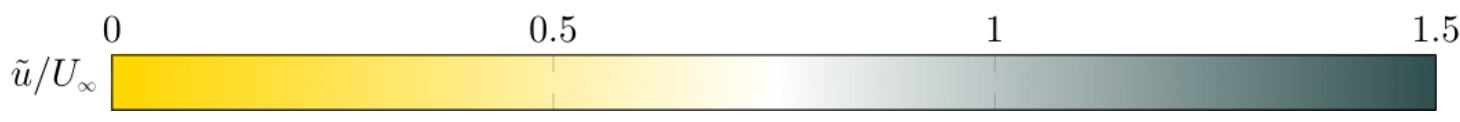

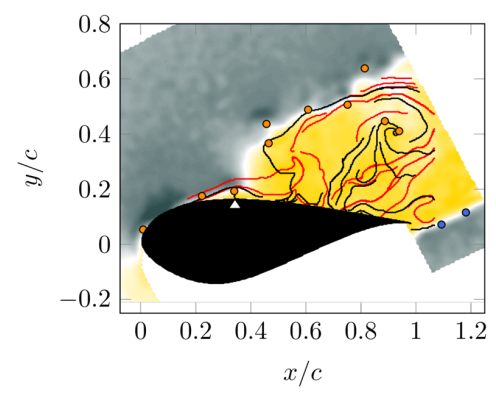

(a) $\alpha=22.26^{\circ}, \tau=104.26$

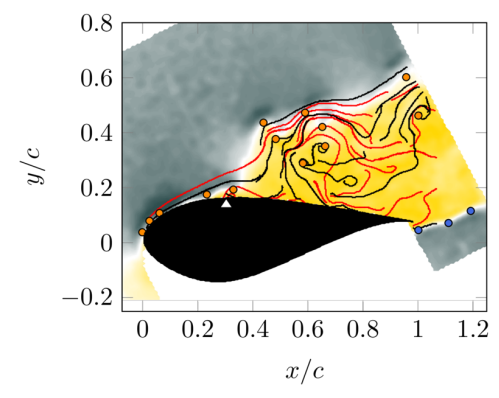

(d) $\alpha=22.11^{\circ}, \tau=105.07$

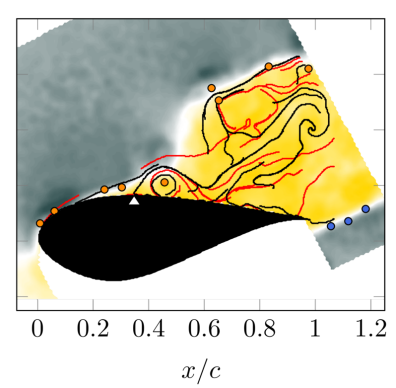

(b) $\alpha=22.21^{\circ}, \tau=104.53$

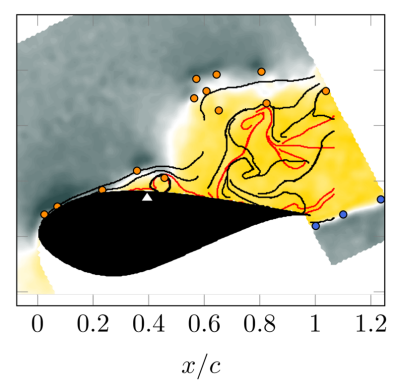

(e) $\alpha=22.06^{\circ}, \tau=105.33$

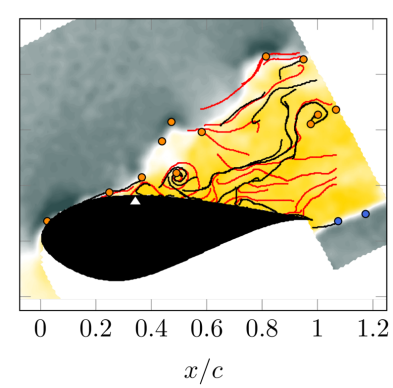

(c) $\alpha=22.16^{\circ}, \tau=104.80$

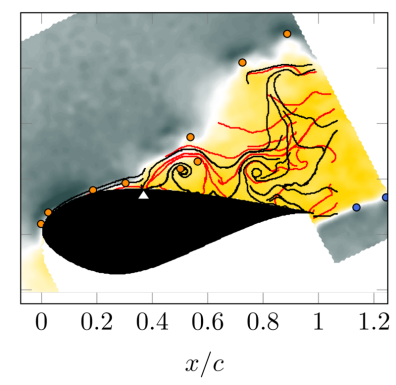

(f) $\alpha=22.01^{\circ}, \tau=105.60$

Figure 5.4: Instantaneous velocity fields over six consecutive PIV snapshots during reattachment, plotted with attracting (black) and repelling LCS (red), separation point $(\Delta)$, and locations of clockwise (orange) and counter-clockwise (blue) rotating vortex core locations.

are represented in grey and negative fluctuations in yellow. The first EMD mode represents the highest frequency and lowest amplitude spatial signals extracted using EMD. These small scale events are predominantly present in regions of high shear. The spatial dimension between EMD peaks in mode one are found to be $3.4 \mathrm{~mm}$ or $\approx 2 \%$ of the chord on average. When compared to the dimension of a vortex, the scale is approximately the radius of a shear layer vortex. There is undoubtedly a correlation between the location of a vortex and the surrounding EMD 


$\begin{array}{rllllllllll}-0.1 & -0.08 & -0.06 & -0.04 & -0.02 & 0 & 0.02 & 0.04 & 0.06 & 0.08 & 0.1 \\ u^{\prime} / U_{\infty} & & & & & & & & & \end{array}$

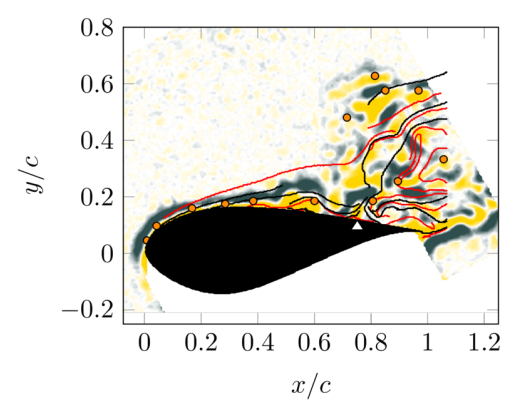

(a) $\alpha=22.16^{\circ}, \tau=52.27$

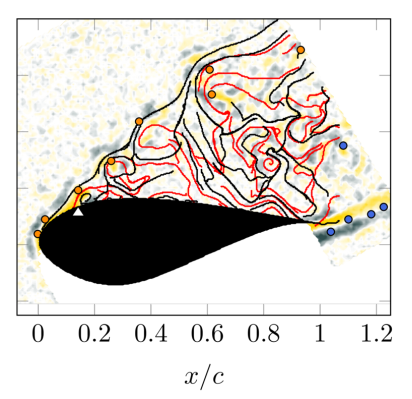

(b) $\alpha=24.0^{\circ}, \tau=86.67$

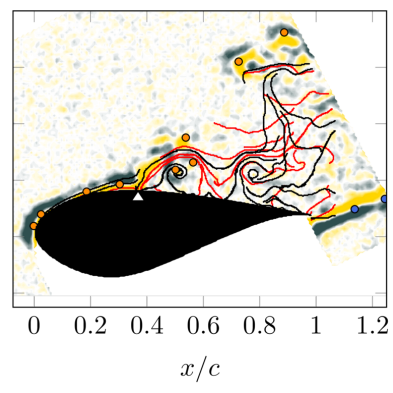

(c) $\alpha=22.01^{\circ}, \tau=105.60$

Figure 5.5: First empirical mode for separation (a), full stall (b), and reattachment (c).

peaks. In each case, the rotational sense of the vortex acts to either accelerate or decelerate the flow relative to the free stream. This is shown in figure 5.5(a) at $x / c=0.8$, where the EMD peak is positive between the counter rotating vortices. Also of note, is the upstream positive EMD peak followed directly downstream by a negative peak at the point of separation. This pattern is found in close proximity to the surface vortex nearest to the trailing edge. The location of vortices in the first EMD mode in predominantly found along EMD zero contours, directly between positive and negative peaks. During full stall and reattachment, there are almost no EMD peaks within the stalled region. This differs from the developing stall case, where the majority of the stall region is comprised entirely of EMD peaks. The first EMD mode shows no clear connection to the LCS, which is attributed to the relative scale difference between the small scale fluctuations and the coherent structure which persist over larger spatial scales.

Figure 5.6 depicts the second EMD mode from the selected images and is con- 


$\begin{array}{rllllllll}-0.2 & -0.15 & -0.1 & -0.05 & 0 & 0.05 & 0.1 & 0.15 & 0.2 \\ u^{\prime} / U_{\infty} & & & & & & & & \end{array}$

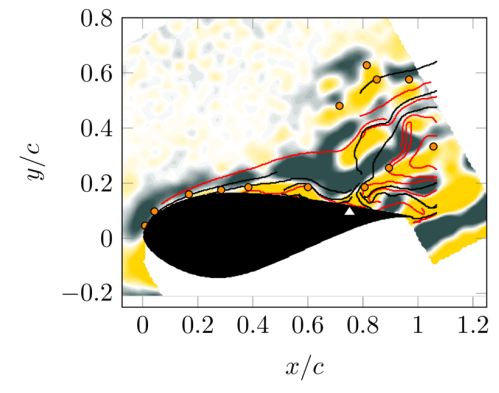

(a) $\quad \alpha=22.16^{\circ}, \tau=52.27$

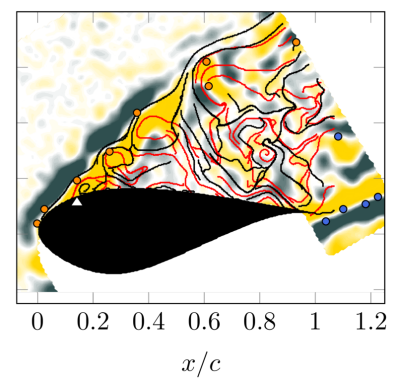

(b) $\alpha=24.0^{\circ}, \tau=86.67$

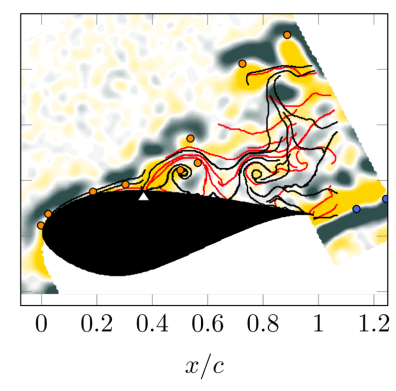

(c) $\alpha=22.01^{\circ}, \tau=105.60$

Figure 5.6: Second empirical mode for separation (a), full stall (b), and reattachment (c).

nected to the previous results through the computed LCSs. The spatial scales have increased to $8 \mathrm{~mm}$ or $0.05 \mathrm{c}$ on average and on the same order as the LCSs. Also the occurrence of a negative or positive EMD peak is correlated to the orientation and presence of repelling and attracting LCSs, respectively. The connection between vortices in the shear layer is similar to the first mode, where vortices are found between strong positive and negative peaks. The primary shear layer attracting LCSs in these figures are found along the border between positive and negative EMD peaks. The existence of a surface vortex is seen to create a upstream negative EMD peak, which is interpreted as an obstruction to the surface flow. The location of the separation point is also apparent within the EMD results for mode two, which is regularly a negative peak.

Figure 5.7 shows the spatial scales extracted as the third EMD mode for the seleted data. Spatial scales have increased to the order of two vortex diameters, approximately $0.2 c$. The dominant features that are apparent in mode three are 


$\begin{array}{rllllllll}-0.2 & -0.15 & -0.1 & -0.05 & 0 & 0.05 & 0.1 & 0.15 & 0.2 \\ u^{\prime} / U_{\infty} & & & & & & & & \end{array}$

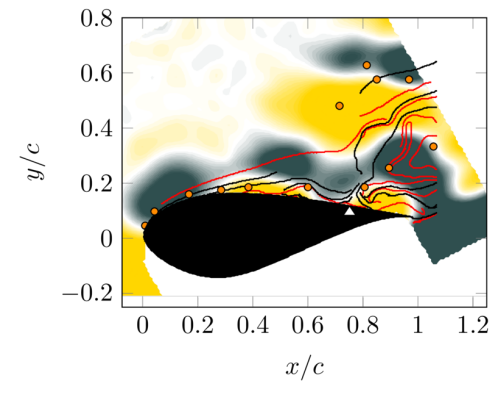

(a) $\quad \alpha=22.16^{\circ}, \tau=52.27$

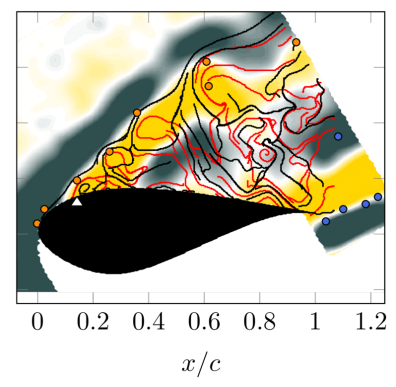

(b) $\alpha=24.0^{\circ}, \tau=86.67$

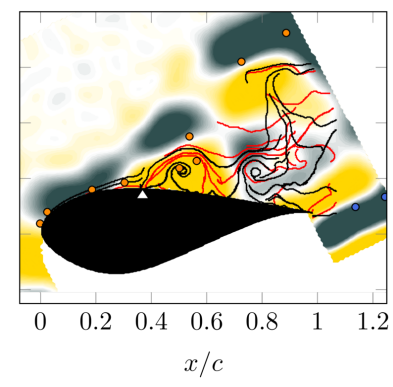

(c) $\alpha=22.01^{\circ}, \tau=105.60$

Figure 5.7: Third empirical mode for separation (a), full stall (b), and reattachment (c).

the separation point, the shear layer, and the area of vortex interactions within the separation region, represented by negative velocity fluctuations. During separation in figure 5.7(a), the location of low velocity peaks are primarily confined to the shear layer and regions with surface vortices. A pattern emerges in mode three as locations that contain packets of attracting LCSs are often positive EMD peaks. This is presented in figure 5.7(b) at $x / c=0.6$ directly above the surface. In mode 3 , the locations of positive peaks and negative peaks are linked to the curvature of the local LCS, similar results are found in [77] where curvature or lack thereof in the LCS corresponds to the acceleration or deceleration of the flow, respectively. Negative peaks contain hyperbolic LCS which are highly curved and often contain crossings of attracting and repelling LCS. On the other hand, regions of the EMD with positive peaks contain straight and often parallel LCS.

The residual signal provided by mode four shows the mean flow behavior. The wake region and the free stream are depicted. The separation point is also shown in 


$\begin{array}{ccccccccccc}0.75 & 0.8 & 0.85 & 0.9 & 0.95 & 1 & 1.05 & 1.1 & 1.15 & 1.2 & 1.25 \\ u^{\prime} / U_{\infty} & & & & & & & & & & \end{array}$
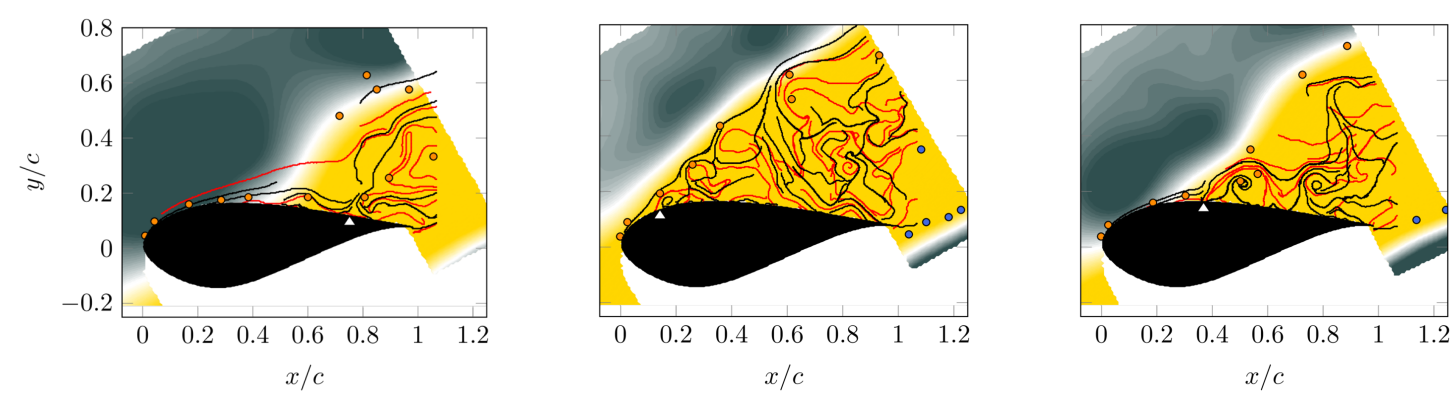
(a) $\alpha=24.31^{\circ}, \tau=0.354$
(b) $\alpha=24.36^{\circ}, \tau=0.356$
(c) $\alpha=24.41^{\circ}, \tau=0.358$

Figure 5.8: Fourth empirical mode for separation, full stall, and reattachment.

this mode. Based on these observations, the EMD resultant captures information about spatial scales which envelope the wake flow. Throughout the experiment, the typical spatial scale represented by the residual is approximately $60 \mathrm{~mm}$ or $0.4 c$.

\subsection{Conclusions}

Unsteady flow characteristics of stall development and reattachment are captured using TR-PIV. Serving to determine the relationship between spatial scales identified by empirical mode decomposition and time dependent behavior of the separation point and shear layer during these transitions. Using LCS, the shear layer and separation points are defined as time dependent events and evaluated in short time series by comparing to the corresponding EMD scales of velocity fields.

Initial evaluation of the separation point during transition showed fluctuations of more than 30\%. The application of LCS identification depicts the separation point via the convergence of attracting material lines inside the separated area, supporting 
theoretical considerations of [18]. The shear layer is consistently represented by an attractor that would originate at the airfoil surface and extend over the remaining portion of the chord. Through the additional computation of vortex centers using the $\Gamma$-criterion, the shape of the LCS within close proximity of the vortex are distorted following the rotational sense of the vortex. The relationship between nearby vortices and the shear layer LCS brought to light that the residence of a vortex formed at the separation point has the potential to force the shear layer attractor to the airfoil surface. This is shown during stall development, where a secondary separation point is formed closer to the trailing edge despite a change in the angle of attack of less than $\Delta \alpha \leq 0.3^{\circ}$. The emergence of a vortex which remains near the surface for an extended period of time is expected to have significant impact on the aerodynamic forces experienced by the blade, as long at it remains near the surface.

The role of a vortex formed at the separation point is the focus of the remaining LCS analysis. During full stall, the shear layer vortex typically causes small scale perturbations of the shear layer LCS, which is amplifying while traversing downstream. The amplification and vortex distribution in the shear layer is influenced by the internal flow behavior in the separation region. The separated flow region is divided into two portions by a group of parallel LCS attractors. The inner separation area, near the point of separation is dominated by regular vortex shedding and shear layer perturbation patterns. The second area is deemed a recirculation zone, characterized by hyperbolic LCS behavior and large scale shear layer perturbations.

During reattachment, surface vortices once again draw the shear layer LCS towards the airfoil surface. The convection of a separation point vortex away from 
the surface is followed by a reaction of the separation area, which is indicated by a large inflection in the shear layer LCS and the return of the separation point to a position closer to the leading edge. During reattachment however, the existence of a surface vortex did not create a secondary separation point and thus results in smaller changes in the separation point of $\Delta f \leq 10 \%$ relative to the large changes in the separation point, $\Delta f \geq 30 \%$, observed during stall development.

Next, the PIV fields are analyzed by means of an empirical mode decomposition to identify the characteristic spatial scales for each of the three stages discussed previously. The EMD spatial scales are related to the size of the shear layer vortices. The relationship between the EMD peaks and the shear layer vortices are found to be consistent within the first three modes. The latter also reside along EMD zeros between positive and negative peaks. This result is directly attributed to the rotational influence upon the velocity field surrounding the vortex.

The separation point is shown in each mode; this supports the conclusion that the separation point is the origin of the dominant flow structure during unsteady flow separation. Near the separation point, a positive EMD peak exists upstream of the separation point and a negative peak exists at the point of separation. For the first EMD mode, the areas with the largest concentration of peak fluctuations are found in regions of high shear. During a fully stalled state, the interior of the separated flow has relatively small EMD peaks despite the significant recirculation that is occurring in this area. During transition, the distribution of EMD peaks varies considerably between measurements, giving insight into the chaotic nature of transition. The first EMD mode peaks are spatially determined to be on the order of a vortex radius based on the $\lambda_{2}$ criterion. 
In relation to the LCSs, the primary shear layer attractors in the second EMD mode run along the boarder between positive and negative EMD peaks, represented in stable flow states such as the fully separated state. The location of the surface vortex in the second EMD mode is found to be consistently near a negative EMD peak, showing that the vortex itself is connected to lower flow velocities. The spatial scales of the second mode peaks are on the order of the $\lambda_{2}=0$ contours, making these scales close to the area of influence of the shear layer vortices.

Results from the third EMD mode are found to describe flow structures that are closely connected to the LCS trends. In areas where LCSs lie parallel to adjacent LCSs and are relatively straight, the EMD consistently demonstrates positive peaks. The opposite is true for areas where groups of parallel LCSs are highly curved and negative EMD peaks are observed. The spatial scales of the EMD are found to encapsulate recirculation zones and surface vortices, demonstrated particularly well in the primary shear layer LCS perturbation amplitudes.

The fourth EMD mode represents the bulk flow structures such as the stall regions in each stage where flow has slowed down noticeably. This region is considered to be the lowest order approximation of the instantaneous flow behavior, but does not offer significant information about the internal flow structures. The most significant contribution of the fourth EMD mode is that it provides the integral length scale, which is applied during the calculation of the LCS.

The EMD modes are put to use in selecting the characteristic velocity and integral time scales for the LCSs in each stage of the unsteady separation and reattachment process. Through the use of these techniques, the influence of surface vortices which form at the separation point is shown in detail throughout the stall cycle. 
This result motivates future work in which these techniques can be used to outline vorticity transport during transitions. 


\section{The critical role of surface vorticity during unsteady separation}

As vorticity builds up, it obstructs the flow along the blade surface deflecting the free stream away from the surface increasing the downstream velocity deficit, effectively increasing the boundary layer thickness. At some point, the low momentum fluid within the boundary layer is unable to overcome the adverse pressure gradient downstream and is ejected into the outer flow. This is accompanied by flow reversal generated by the surface vorticity, which acts to reduce surface friction. Should the influence of the adverse pressure gradient become strong enough, the friction at the wall will drop to zero, $\tau_{w}=\partial u / \partial y=0$, at which point flow will reverse and steady separation occurs according to [9].

When a moving fluid encounters a solid surface, such as an airfoil, viscous forces act to rapidly decelerate the flow near the surface in a thin boundary layer. The viscous interaction near the blade surface introduces circulation and vorticity into the flow. According to Prandtl's separation conditions for steady flow past a twodimensional streamlined body, flow will separate from the surface, where the skinfriction is reduced to zero and a negative gradient exists. This gives a Eulerian description of boundary layer behavior, which fits well in the case of steady sepa-

ration [9]. However, this criterion breaksdown in unsteady flow separation leading 
to a singularity in the steady boundary layer solution when the wall shear stress vanishes, first discovered by [10]. The Goldstein singularity was studied simultaneously by [11], [12], and [13], who independently arrived at the conclusion that vanishing wall-shear stress and the accompanying flow reversal near a wall do not necessarily guarantee flow separation under unsteady conditions, known now as the MRS criterion. According to the MRS criterion, the separation point must occur on the zero vorticity line bisecting a recirculation region.

To address the uncertainty associated with unsteady separation resulting from the MRS criterion, a Lagrangian solution was proposed by [14]. The Lagrangian approach was found to accurately describe the convergence and ejection of fluid particles from an unsteady boundary layer flow. The Lagrangian formulation for the unsteady boundary layer has two advantages: (i) it decouples the motion in the streamwise direction from that in the normal direction and (ii) an objective criterion for boundary layer separation. To improve future predictive capabilities of the onset and duration of unsteady separation, the role of vorticity in shear layer stability is considered. In an exposition of shear layer vorticity during separation, [24] describe that the shear layer experiences spatial perturbations developed via KelvinHelmholtz instability, which scale with the thickness of the separated flow region, growing in magnitude as they are convected downstream [24]. Recalling that the growth of the shear layer perturbations leads to inflections of the shear layer directly downstream of the separation point and abrupt movements of the separation point itself [26]. The shear layer perturbation amplitude at the point of separation is expected to have a direct effect on the eruption of near-wall vorticity away form the surface and into the outer flow, a hallmark of unsteady separation according to [23]. 
Despite the advances in the theoretical development of unsteady separation, there is still a lack of experimental evidence with respect to the Lagrangian interaction between the vorticity within the boundary layer and its role within the context of unsteady separation. In various engineering applications, the remaining challenge is to identify the initial location and strength of separating shear layers [27]. The results of this study describe a critical role of surface vorticity accumulation in unsteady separation and reattachment. Thorough evaluation of the unsteady characteristics of the shear layer reveal evidence that the build-up and shedding of surface vorticity directly influence the dynamic changes in separation point location. To observe the direct impact of the vortex shedding frequency and accumulation of vorticity on the perturbations of shear layer, the $\lambda_{2}$-criterion provided by [56] and the $\Gamma_{2}$-criterion from [58] are employed over each velocity field. By definition $\lambda_{2}$ is Galilean invariant, but is inherently a non-objective definition. Evaluation of the shear layer stability herein includes calculating the tortuosity and displacement amplitudes the shear layer. Tortuousity is used to capture the instability of the shear layer by describing the amount it contorts during unsteady separation, it has been used in describing porous media [78] or the curvature of circulatory systems [79].

\subsection{Results}

In Figure 6.1(a), the DU 99-W-350 profile is plotted on normalized axis where chordwise, $x$, and azimuthal, $y$, directions are normalized by the chord length. Figure 6.1(a) presents a comparison between the shear layer attacting LCS (black), as defined by [71], and a shear layer is identified by a velocity ratio of $U_{s l}=0.48 U_{\infty}$ (red). Vortices are identified using the $\Gamma_{2}-$ criterion, clockwise (orange) and counter- 
clockwise (blue), respectively. Each shear layer is plotted over the instantaneous vorticity field to show the relative ability of each to capture the behavior of the shear layer vortices and the respective vorticity properties during the stall cycle. The separation point, determined by locating the first instance of negative streamwise velocity near the airfoil surface, is shown as a white $\Delta$. The velocity ratio originates just upstream of the point of separation and falls directly between two merging attracting LCS, which extend away from the surface directly above the separation point. The two shear layer identifiers lie on top of each other until approximately $x / c=0.5 c$. At this point, the velocity ratio based shear layer approximation proceeds along the shear layer vortex cores. The maximum amplitude of each of the computed shear layers are nearly equal. The advantage of the velocity ratio is that it is computationally inexpensive and does not require time resolved data to capture accurate shear layer behavior within a PIV framework.

Vorticity $(\omega)$, when normalized $\omega^{*}=\omega c / U_{\infty}$, reveals a minimum peak value at or near the separation point. This value is repeatedly found to be $\left|\omega^{*}\right| \geq 20$. Above this value, the localized vorticity entrains enough fluid near the surface to create a flow reversal which results in the vortex to "leap" off to the surface. Once the shear layer is captured, the vorticity fields is extracted along the contour. The results provide the spatial distribution of normalized vorticity peaks, $\left|\omega^{*}\right|_{\max }=\left|\omega c / U_{\infty}\right|_{\max }$, along the shear layer downstream of the separation point, where $\omega$ is the vorticity, $c$ is the chord length, and $U_{\infty}$ is the free stream velocity. An example of this is provided in figure 6.2. The shear layer approximation is plotted in dark red on top of the corresponding vorticity field. Vorticity peaks, $d \omega^{*} / d x=0$, along the shear layer are represented by white circles and the magnitudes for each peak are plotted 


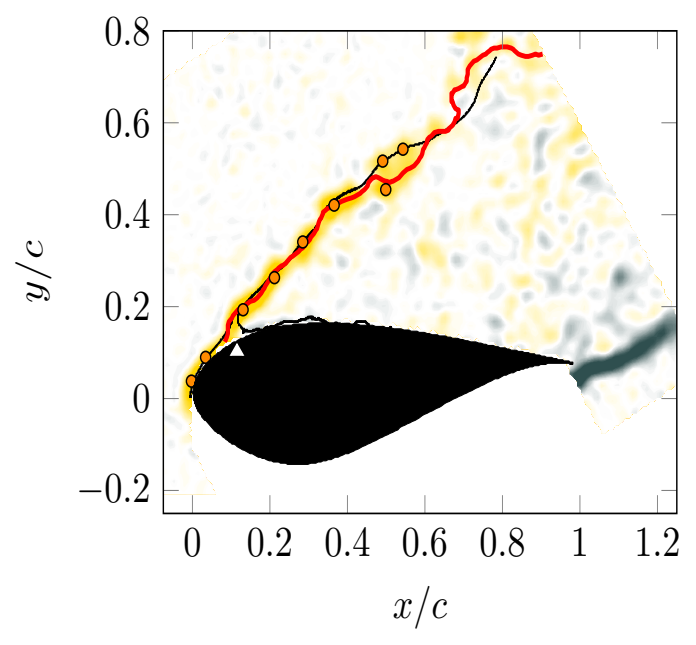

(a)

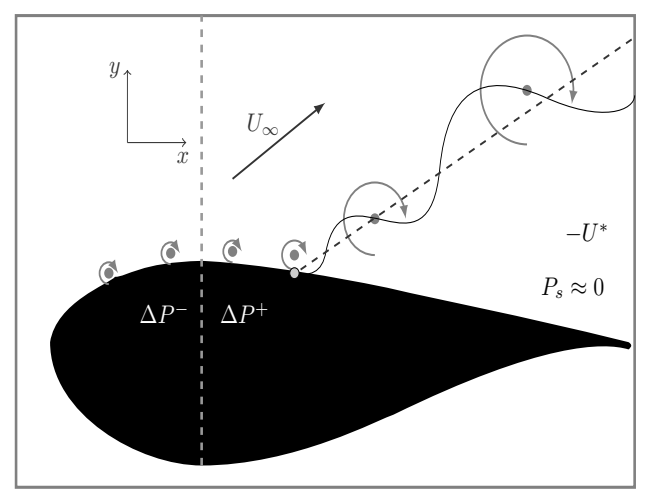

(b)

Figure 6.1: (a) Velocity ratio $U_{S L}=0.48 U_{\infty}$ (red) plotted with the shear layer attracting LCS (black) on top of the instantaneous vorticity field during full stall at an angle of $\alpha=24$ (b) Schematic of theoretical unsteady separation over an airfoil. For consistency of presentation, coordinates are rotated to chord based coordinates $(x, y)$, adjusted for the angle of attack, $\alpha$, relative to the freestream velocity, $U_{\infty}$. The perturbed shear layer is plotted as a black line emanating from the separation point (grey 'o') with perturbations caused by departing surface vortices (grey ' $\rightarrow$ ') plotted over a black dashed line which represents a steady shear layer without perturbation.

on the lower $y$-axis which has the absolute value of vorticity as a function of $x / c$ location. To capture the perturbation amplitude, $A$, a linear fit is applied to the average of the first 26 data points of the measured shear layers within the interval of $\pm 5 \tau$, where $\tau$ is the convective time scale $t U_{\infty} / c$. This average linear fit is taken as an approximated shear layer zero line, plotted here as a black dashed line. An example of the perturbation growth in time and space, the shear layer at $\left(\tau_{1}\right)$ and $\left(\tau_{2}\right)$ are plotted in red and light red, respectively. The trend of vorticity extrema along the shear layer is shown in figure 6.2. At the point of separation, there is a peak vorticity which in normalized value exceeds $\left|\omega^{*}\right|_{\max }>40$. The vorticity 


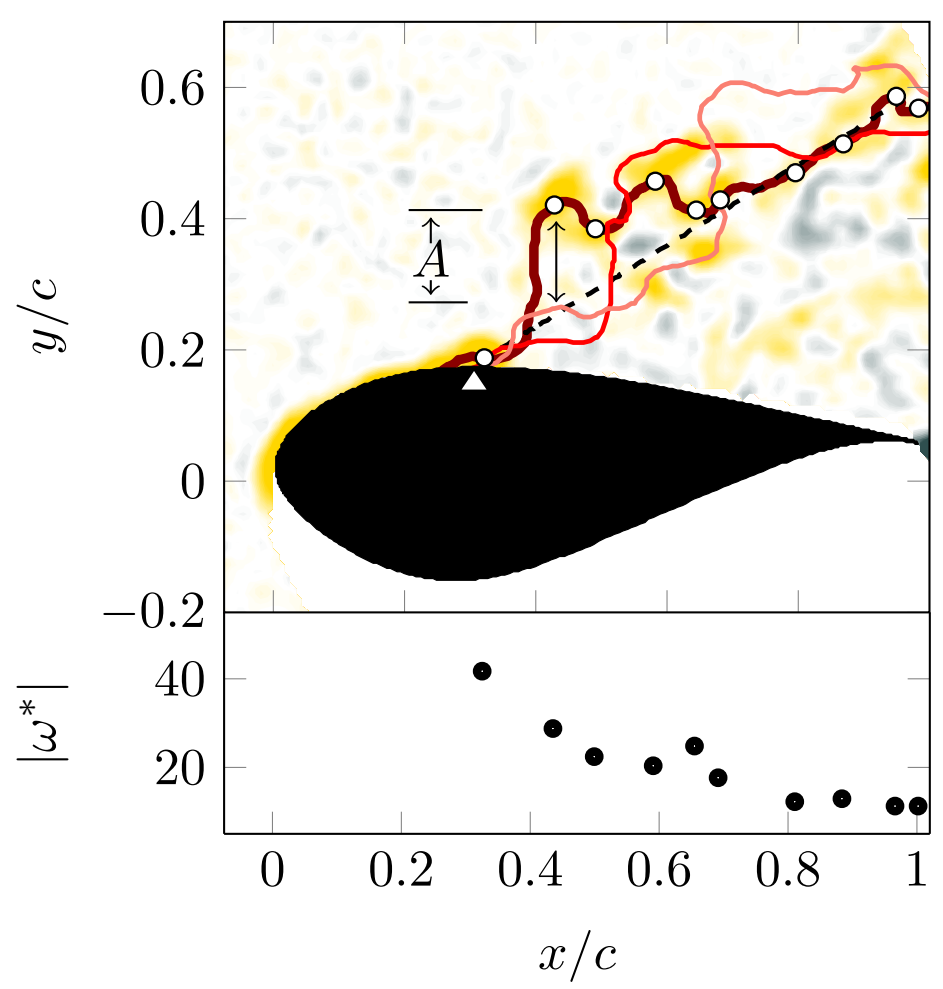

Figure 6.2: (a) Example of shear layer stability during reattachment. Three sequential shear layers plotted as $\tau$ (dark red), $\tau_{1}$ (red), and $\left(\tau_{2}\right.$ (light red) along a mean shear layer (black dashed) based on the shear layers of $\pm 5 \tau$ with $A$ as the perturbation amplitude. On the lower axis, normalized vorticity maxima, $\left|\omega^{*}\right|$, taken from along the shear layer, plotted as function of chordwise position $(x / c)$.

peaks, where $d \omega^{*} / d x=0$, along the shear layer are shown to decay exponentially with increasing $x / c$ location. Using this technique to evaluate each snapshot in the data set, consistently reveals that the vorticity formed at the separation point is greater than $\left|\omega^{*}\right|_{\max }=20$ and is larger in magnitude than the vorticity which has been convected away from the surface. This indicates that there is a critical level of vorticity which must accumulate in order for separation to occur.

Using the techniques from [76], the transitional stages within the stall cycle 


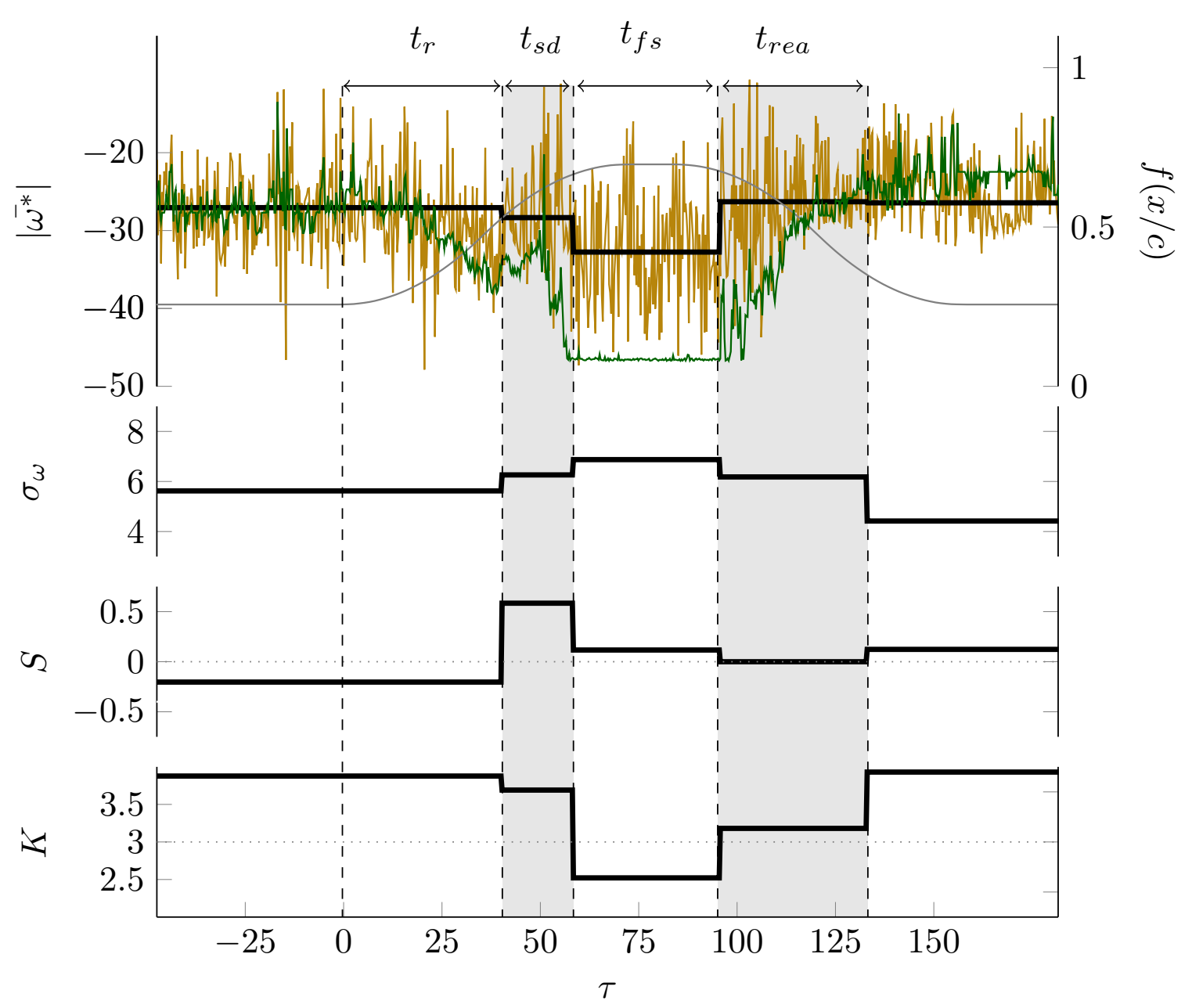

Figure 6.3: Peak vorticity at the point of separation (gold) plotted as a function of $\tau$, plotted along side the separation point (green) and the motion profile (grey), with the mean $\left|\omega^{*}\right|$, standard deviation, skewness, $S$, and kurtosis, $K$, plotted as functions of time for the selected stall stage at $z / R=0.317$ at a pitch rate of $\dot{\alpha}=17.14$

are identified. The separation point, $f$, which is determined by locating the first occurrence of a negative streamwise velocity near the airfoil surface, is plotted as a function of time in figure 6.3. Here, the separation point is plotted (green) with the angle of attack (grey) for the $y$-axis and both are plotted as a function of 
normalized convective time $\tau=t U_{\infty} / c$. The intervals that capture stall development and reattachment are highlighted in gray in figure 6.3.

To evaluate the trend of the vorticity accumulation at the point of separation, the first vorticity peak along the shear layer is taken from each time step. In figure 6.3, the first four statistical moments of the first vorticity peak downstream of the separation point, $\left|\omega^{*}\right|$, are presented. The top plot includes the separation point (green) and the angle of attack (gold) for reference and the mean peak vorticity in black calculated for each of the identified stall stages (delineated by vertical dashed lines) as functions of time. The time intervals describe the duration of each stage of the stall cycle. The first time scale is the reaction time, $t_{r}$. This time interval begins with the initial motion of the blade and the end is marked by a dramatic change in behavior of the separation point. The second time interval is termed the stall development time scale and is represented by $t_{s d}$. The beginning of this stage is marked by the emergence of stall development and a change in the separation point and ends when the primary stall vortex is shed, corresponding to a second dramatic change in the separation point. The change marks the beginning of the next interval, identified as the period of time that the blade is experiencing full stall, $t_{f s}$. The phase ends when the angle of attack is reduced and the flow begins to reattach. During this process, the separation point moves back downstream from the leading edge. The fourth and final time interval is the time required for the flow to reattach to the surface and is termed the reattachment time scale $t_{r e a}$. The mean value during attached flow, prior to separation, is $\left|\bar{\omega}^{*}\right|=25.9$. The mean increases by $8 \%$ to $\left|\bar{\omega}^{*}\right|=28.0$ during stall development. Once the flow enters full stall, the vorticity peak increases to $\left|\bar{\omega}^{*}\right|=32.6$, a $25 \%$ increase from the attached flow mean. 
The peak vorticity drops to $\left|\bar{\omega}^{*}\right|=25.5$ during flow reattachment. After the stall cycle is complete, the attached flow vorticity is the lowest of the calculated means at $\left|\bar{\omega}^{*}\right|=25.1$. As the angle of attack increases, the vorticity accumulates within a smaller region and has a smaller diameter relative to the accumulated vorticity at lower angles.

The standard deviation is presented below the mean. Peak vorticity by stage shows a standard deviation that varies by stage from $\sigma=5.4,5.9,7.1,6.1$ to 4.4 , an increase of $30 \%$ from attached flow during full stall. The trend in the standard deviation represents the variability amongst larger peak values, generated during transition to full stall and during full stall. The decrease in the standard deviation during reattachment and attached flow is due to the relatively lower values of peak vorticity shedding for these stages.

The skewness, $S$, of the vorticity distribution describes the positive or negative bias within the excursions from the mean. During the attached flow states prior to and after the blade motion is $S=0.1$, showing a slightly lower tail which is lower than the mean. Once stall onset begins, the skewness increases to $S=0.59$, showing more deviations lower than the mean. Once in full stall, $S$ drops to 0.25 as the distribution trends more towards a Gaussian distribution during fully developed stall states. Perhaps the most intriguing skewness trend is seen during the transitions between attached flow and full stall. As the angle of attack is reduced and the flow begins to reattach, the sign and magnitude of the skewness changes to -0.08 . The change of sign here indicates that the peak vorticity is experiencing larger excursions of the peak vorticity above the mean. Once flow reattaches, the skew for the vorticity peak becomes positive again with $S=0.14$. The general trend of the skew is to 
have positive peak values and large excursions that are less than the mean. The only time that the trend reverses is during reattachment. Physically, this is explained by the vorticity packet spreading into larger areas as the adverse pressure gradient is reduced. As a consequence, the circulation of vorticity is reduced at the separation point during the reattachment, which causes the mean peak vorticity to drop. Thus, the build up and shedding of the vorticity packets requires a lower peak on average but has occasional excursions above the mean.

The fourth statistical moment presented is the distributions kurtosis, $K$. Kurtosis, describes flatness or the range of the probability distribution in terms of excursions from the mean. For a perfectly Gaussian distribution, the kurtosis is equal to 3. For the attached flow segments of the stall cycle, the kurtosis for peak vorticity is in slight excess at $K=3.15$ and $K=3.13$ for pre-stall and post-stall attached flows, respectively. During flow separation, the kurtosis reaches $K=3.4$, reinforcing that the build-up and shedding of peak vorticity is unstable during transition. Only during full stall does the kurtosis drop below 3 to $K=2.8$ representing a stable formation and shedding of peak vorticity in a fully separated state. As the flow reattaches, the kurtosis reaches an excess of $K=3.14$, indicating slightly larger excursions from the mean than during full stall.

Returning to the perturbation behavior during unsteady separtion, the tortuosity of the instantaneous shear layer is presented, in figure 6.4. Tortuosity is defined as the ratio of the length of the shear layer relative to the distance it covers. In the case of a straight line the tortuosity would be $T=L / D=1$. It follows that a value of $\mathrm{T}$ greater than 1 represents a perturbed shear layer. The tortuosity of each shear layer from the stall cycle is presented in figure 6.4 (green). In the pre-stall attached 


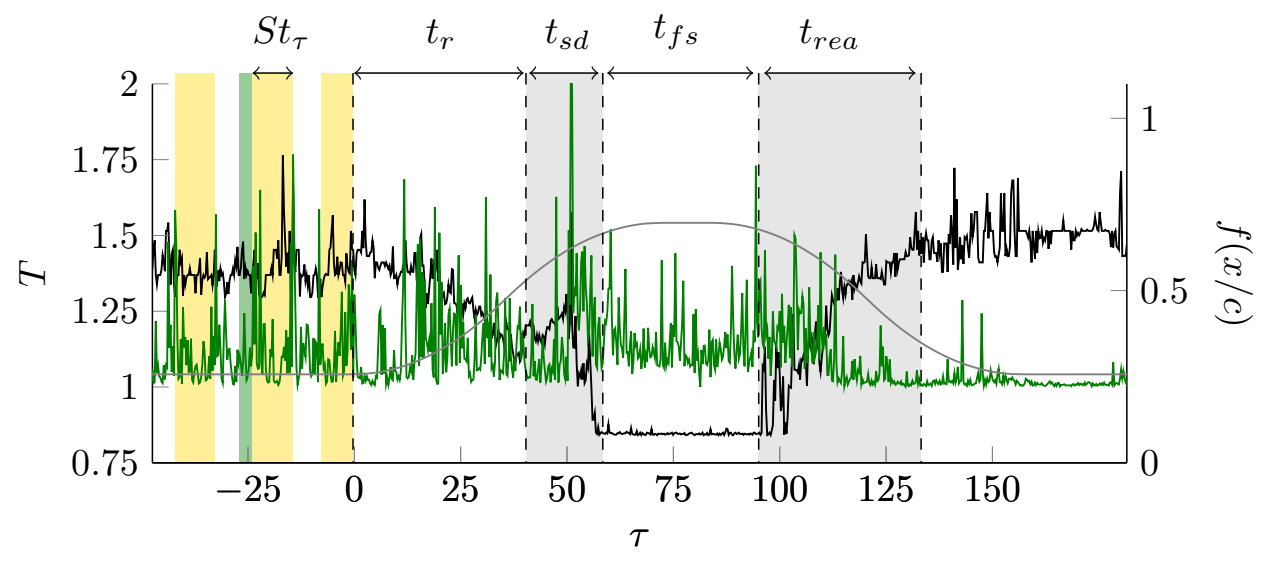

Figure 6.4: Shear layer tortuosity (green) plotted with separation point (black) and pitching motion(grey).

flow, there is an apparent periodic peak in $T$ as it varies between a minimum of $T \approx 1$ and peaks of $T=1.6-1.75$. Along this line, the regularity of the peaks is approximately $\tau=10$. Based on the representation of shear layer perturbations as vortex shedding, the time scale between peaks of $T$ are converted to the shear layer Strouhal number, $S t^{*}=(\Delta \tau) c / U_{\infty}=0.12$, consistent with results found in [80]. The periodic behavior of $T$ is also reflected in the separation point, which has the same frequency but is out of phase by $90^{\circ}$. The out of phase trend continues even when the periodic frequency is no longer apparent. Once the pitching motion starts, the tortuosity increases in volatility and the periodic pattern observed previously is no longer present. The largest average values of $T$ are found in the stall development stage as flow passes through the most unstable state transitioning to full stall. The shear layer is particularity tortuous during the moments just before full stall is achieved. During full stall, the average tortuosity increases, which corresponds to the increase in relative size of the separation region, it is also noted that $T$ is relatively constant throughout this stage. As the reattachment stage begins, there 
is a spike in the tortousity and the variability of $T$ increases. There is a significant drop off in $T$ near $\tau=120$ at which point tortuosity of the shear layer approaches unity and rarely departs for the remaining duration of the time series.

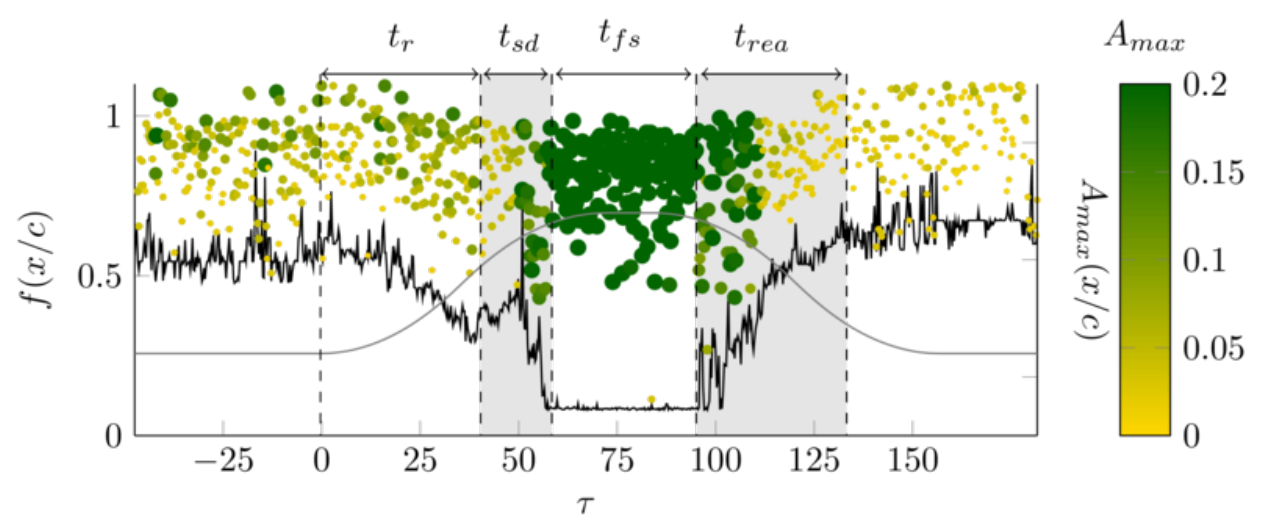

Figure 6.5: Maximum perturbation amplitude vs separation point and pitching motion.

Figure 6.5 provides a deeper look into the peak amplitude of the shear layer perturbation as a function of chordwise position. The instantaneous shear layer is compared to the linear approximation of the mean shear layer to arrive at the maximum perturbation amplitude, $A_{\max }$. Dark green represents larger amplitudes while yellow represents the smaller amplitudes, in $y / c$ values. The peak amplitudes are then plotted along with the angle of attack, $\alpha(\tau)$, in grey and the separation point, $f(\tau)$, in black. The figure shows the relative stability of the shear layer. The general trend during steady angles of attack is that the amplitudes of the shear layer perturbations grow with downstream distance, following the general principles of Kelvin-Helmholtz instability. For example, during the period of time prior to the beginning of the pitching motion, the amplitudes nearest to the point of separation are consistently small, $A_{\max } \approx 0.01$, while the perturbations near the trailing edge 
have grown to $A_{\max } \approx 0.1$ in some cases. As the angle of attack increases and stall development begins, the occurrence of a large $\Delta f$ is accompanied by a large perturbation amplitude that occurs close to the point of separation. This phenomena is shown between $50 \leq \tau \leq 60$ during the largest movements of the separation point. Once the separation process has reached full stall, the maximum amplitudes primarily reside near to the trailing edge. As the angle of attack decreases, the amplitude of the shear layer perturbation grows to values exceeding $A_{\max }=0.15$ and begins to move towards the leading edge. As the flow reattaches, the values of the shear layer peak amplitude drop to values of less than $A_{\max }=0.05$. During this stage, the shear layer roll-up and shedding of the surface vorticity leads to large perturbations at the point of separation. When the vorticity on the surface is shed, the resulting shear layer perturbation is large at the point of separation. As the perturbation is convected downstream, it acts to remove the top portion of the separated volume. It is worth noting that the peak amplitudes of the reattached flow are smaller then what is seen in the attached flow prior to separation.

Figure 6.5 demonstrates that large shear layer perturbations are present near the separation point during stall development and reattachment. To further explore this, the shear layer perturbation peak nearest to the point of separation is analyzed in figure 6.6. It is these particular perturbations which will directly influence the movement of the separation point, according to [26]. The separation point movement increases when perturbations at the separation point are larger than $A_{1} \approx 0.05$. This is most apparent during the initial attached flow, stall development and the initial portion of reattachment, $95 \leq \tau \leq 115$. During full stall and the final attached phase, the perturbation amplitudes are considerably smaller, indicating the relative 
stability of the shear layer during those periods.

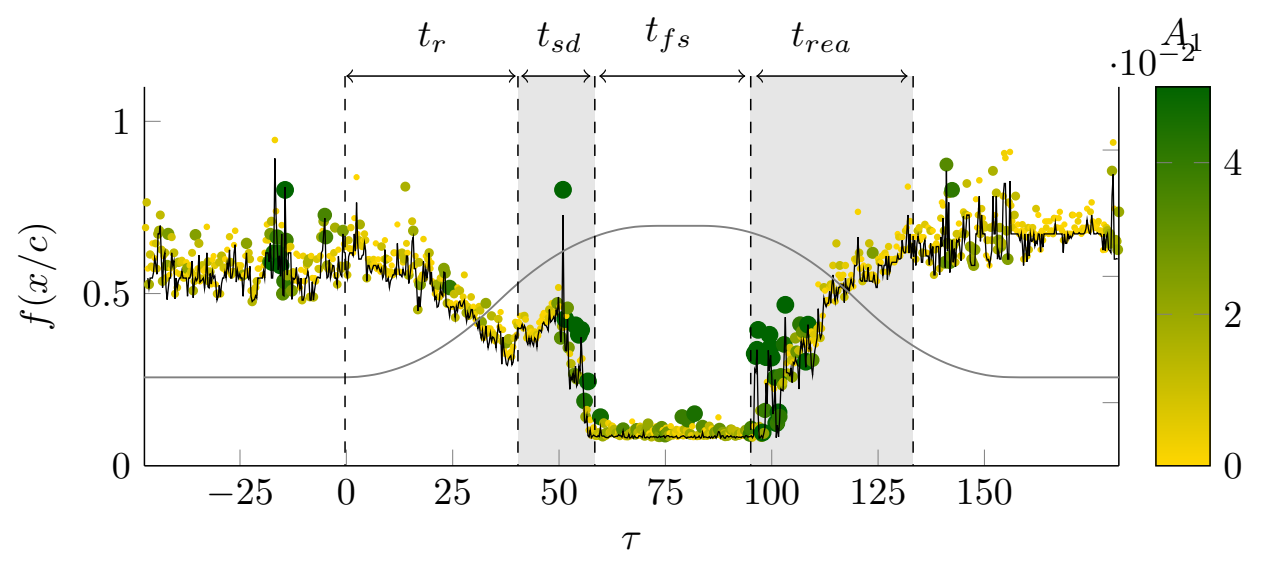

Figure 6.6: Separation point perturbation amplitude vs separation point and pitching motion.

The combination of these results demonstrates that the formation and shedding of a consistent peak vorticity at the point of separation has direct influence on the shear layer behavior and perhaps most importantly the movement of the separation point.

The relationship between the change in the separation point and the strength of vorticity at the surface is tested for Granger causality [81]. Granger causality test results produce F-statistics that imply physical causality. Owing to the specific limitations of a Granger test to stationary signals and that the results are limited to only two of the possible dependent variables in the system, the Granger test does not necessarily imply direct physical causation. However, with a positive test result, it is expected that predictive models for the dependent variable benefit from information included in the independent variable.

Two series of Granger tests are conducted to reinforce the conclusions discussed in the previous section. First, a Granger test is preformed to determine if the 
separation point location results in a change of the peak vorticity. When testing the null hypothesis that the location point does not Granger cause the peak vorticity amplitude, a $p$-value of $10^{-15}$ is the result. The confidence interval being quite

small, the null hypothesis is rejected, and it can be said that information about the separation point location will improve predictions of the peak surface vorticity at the point of separation. Next, the null hypothesis that the vorticity value nearest to the separation point does not Granger cause movement in the separation point is tested. The resulting $p$-value for this test is $10^{-5}$, thus the null hypothesis is rejected and it can be said that the vorticity value at the surface will help to predict the movement of the separation point.

Its implication is that influencing the formation and shedding of the peak vorticity through surface based vorticity control mechanisms could limit the negative impact of the shear layer instability on the movement of the separation point. If successful, this type of surface vorticity control would greatly reduce the unsteady loading caused by unsteady separation.

\subsection{Conclusions}

Using TR-PIV, the role of surface vorticity accumulation in the unsteady movement of the separation point during flow separation is captured. The evaluation of the shear layer stability is quantified via displacement amplitudes and interpolation of the vorticity field along the shear layer approximation. The detection of the shear layer is based on a contour of the convective velocity ratio $U_{S L}=0.48 U_{\infty}$. By evaluating the magnitude of vorticity accumulation at the separation point, there is a critical value of vorticity that is consistently identified at the point of separation. 
This value is repeatedly found to be $\left|\omega^{*}\right| \approx 20$. Above, the localized vorticity entrains enough fluid near the surface to create a flow reversal resulting in the vortex leaping off the surface.

The implication of a consistent vorticity peak at the point of separation is the opportunity to control the extent of separation by influencing the vorticity accumulation over an airfoil surface. It is also observed that immediately following its departure from the surface, the vortex begins to lose strength in an exponential decay. An investigation of the separation vorticity reveals that the first four statistical moments of the vorticity are stall stage dependent. The statistical characteristics of the peak vorticity are correlated to the change in angle of attack. As the pitch is increased, the mean peak vorticity increases. Thereafter, it remains at a larger magnitude during full stall and reduces in magnitude as the angle of attack decreases and flow begins to reattach. This trend is directly related to the angle dependent change in the chordwise pressure gradient, which at higher angles of attack restricts the accumulation area leading to a higher circulation. Evaluation of the standard deviation of the separation point vorticity reveals that during stall development and reattachment, the build up and shedding of vorticity is highly unsteady resulting in larger excursions from the mean value than during attached flow and full stall. The skewness of the separation point peak vorticity is a positive value during all but the reattachment phase of the stall cycle, indicating that the amount of vorticity accumulating at the separation point reaches an upper threshold before separation. During reattachment, the skew changes sign which indicates the reduction in the separation vorticity threshold as the angle of attack is reduced. A stagewise evaluation of the kurtosis suggests that stall development is the most unstable regime of 
the stall cycle.

Separation stability is captured by evaluating the shear layer perturbation amplitude, $A$, and tortuousity, $T$, during the aerodynamic stall cycle. Tortuousity provides a global perspective on the contortion of the shear layer, which passes through stability regimes via the mechanisms of Kelvin-Helmholtz instability during the stall cycle. The growth of shear layer tortuousity leads to the advancement of the separation point. It is shown that during the attached flow, the two markers are out of phase by approximately $90^{\circ}$, with increased toruosity corresponding to the movement of the separation point. The periodic nature of this interaction is linked to the vortex shedding frequency, quantified by a Strouhal number of $S t^{*}=0.12$. The values of $T$ are consistently larger during stall development, full stall and reattachment, owing to the unstable nature of the transitions and convective instability of the shear layer during these stall stages. Looking deeper into the unsteady shear layer behavior, two amplitudes are quantified during the stall cycle. The perturbation maximum location is captured revealing that perturbation amplitudes grow as they are convected downstream, following Kelvin-Helmholtz instability theory. The spatial occurrence of the maximum amplitudes during unsteady separation and reattachment are shown to approach the separation point, leading to an in depth evaluation of the first perturbation peak downstream of the separation point. The result shows that during the stages of stall development and reattachment the shear layer amplitudes are considerably larger than during the more stable full stall and attached flow states.

The combination of these results demonstrates that the formation and shedding of a consistent quantified amount of vorticity at the point of separation has direct 
influence on the shear layer behavior and on the movement of the separation point. The implication is that by influencing the formation and shedding of the peak vorticity through surface based vorticity control mechanisms one could limit the negative impact of the shear layer instability on the movement of the separation point. Such vorticity control would greatly reduce the unsteady loading caused by unsteady separation. These results serve to reinforce the theory of unsteady separation by providing an accurate quantification of the interaction between the accumulation of surface vorticity and the role of the shear layer during unsteady separation. 


\section{Spanwise and pitching rate dependence of peak vorticity and separation characteristics}

\subsection{Peak vorticity statistics}

The evaluation of surface vorticity at near the point of separation is expanded to include the remaining spanwise locations and pitch rates. The discussion begins with the inboard cross-section and thereafter, marching towards the mid-span. Two pitch rates per measurement plane are considered to show the sensitivity of each

profile to wind gust duration. For each of the measurements, the vorticity peak at the separation point is characterized separately, then the results are compared regarding their spanwise position and pitch rate.

The plane located nearest to the root of the turbine blade is a DU 405 aerodynamic profile. This cross-section possesses the largest $t / c$ ratio and has zero added twist. Figure 7.1 shows the peak vorticity plotted against the angle of attack (gold), the separation point (green) and the mean vorticity based on the stall stage (black), all as a function of time. It is shown that the peak vorticity during steady state attached flow average value is $\left|\omega^{*}\right|=20.4$ with a standard deviation of $\sigma_{\omega}=3$. Upon the initial motion, the separation point begins to move towards the leading edge. 


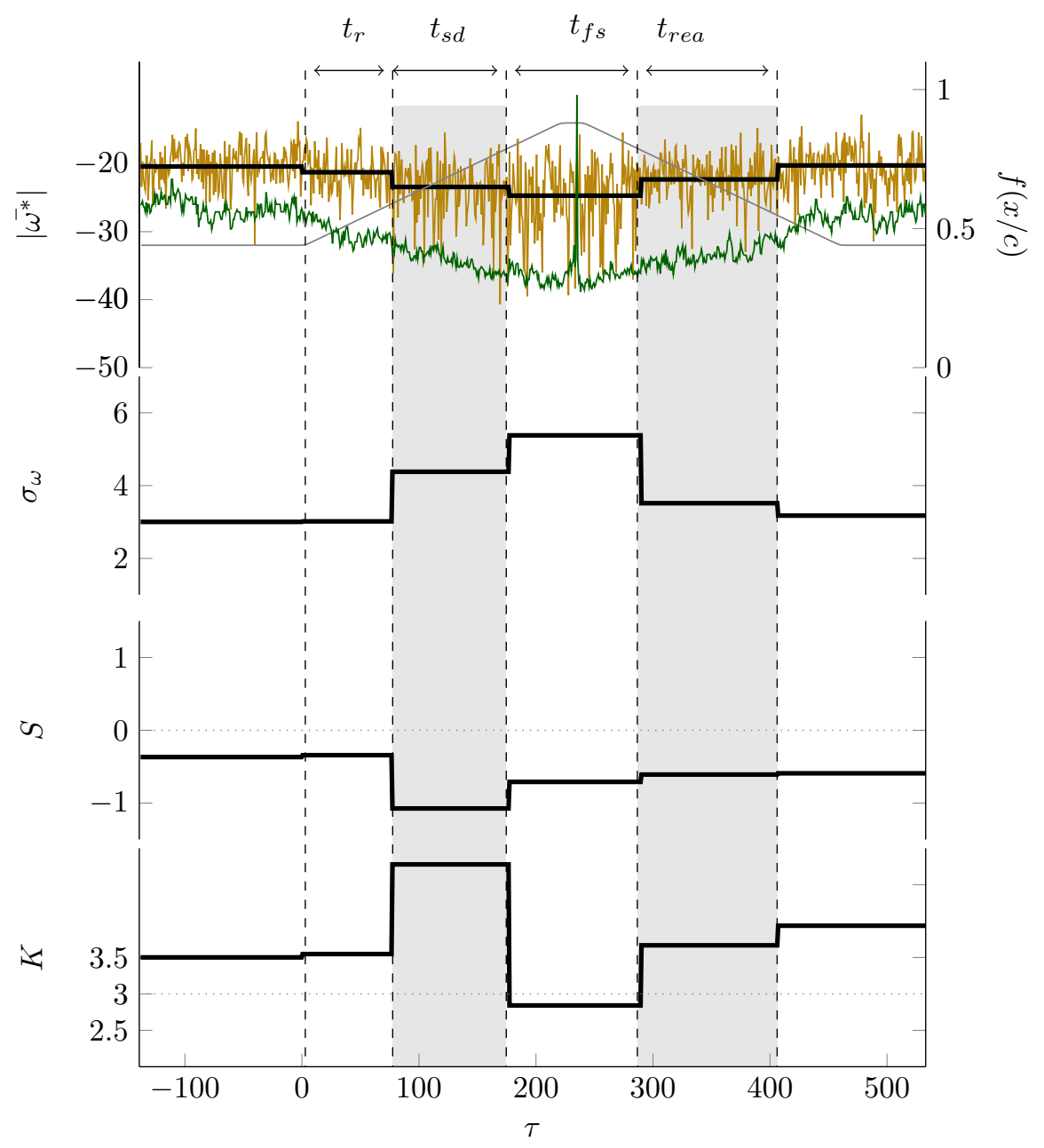

Figure 7.1: Peak vorticity at the point of separation (gold) plotted as a function of $\tau$, plotted along side the separation point (green) and the motion profile (grey), with the mean, standard deviation, skewness, $S$, and kurtosis, $K$, plotted as functions of time for the selected stall stage at $z / R=0.187$ at a pitch rate of $\dot{\alpha}=17.14$.

During this stage, the peak vorticity shows an increase in the mean vorticity and no notable change in $\sigma_{\omega}$. The beginning of the stall development stage is marked by an increase in the standard deviation of $50 \%$ up to $\sigma_{\omega}=4.4$. The peak vorticity during this stage increases to $\left|\omega^{*}\right|=23.4$; a $20 \%$ increase. The cross-section 
enters full stall at an angle of $\alpha=19^{\circ}$. In a fully stalled state, the peak vorticity increases slightly to $\left|\omega^{*}\right|=24.7$, but the variability of the peak increases by $22 \%$ up to $\sigma_{\omega}=5.4$. The peak vorticity during full stall exhibits the lowest kurtosis during the experiment showing a nearly Gaussian distribution. Reattachment begins at nearly the same angle of attack of $\alpha=19^{\circ}$. The reattachment stage demonstrates a drop in the standard deviation, which is $25 \%$ lower than the transition into stall. The kurtosis increases, thus reveling a wider distribution of values. Skewness of the peak vorticity shows a consistently negative value throughout the experiment with a minimum during stall development. The change in skew is an indication that during an increasing angle of attack, the vorticity at the separation point grows beyond the mean.

Figure 7.2 shows peak vorticity from the same cross section as in figure 7.1 , with an increased pitch rate of $\dot{\alpha}=34.28$. The peak vorticity initial magnitude is nearly the same as in figure 7.1. Throughout the experiment, the peak vorticity possesses a mean value unaffected by the pitch rate. It follows a similar trend as seen previously, increasing with angle of attack reaching a maximum value of $\left|\omega^{*}\right|=23.4$, an increase of $30 \%$. The standard deviation reveals a nearly identical distribution, beginning at $\sigma_{\omega}=2.7$ during attached flow, increasing during stall development and reaching a maximum during full stall of $\sigma_{\omega}=5.7$. The skewness of the vorticity peak is again negative throughout the experiment with a minimum during separation, however in the faster pitch rate, the skewness during reattachment is nearly a minimum as well. Kurtosis during transitions reaches a maximum of $K=3.5$, and is otherwise unaffected during the other stages.

Moving away from the root along the span, the next cross-section to be examined 


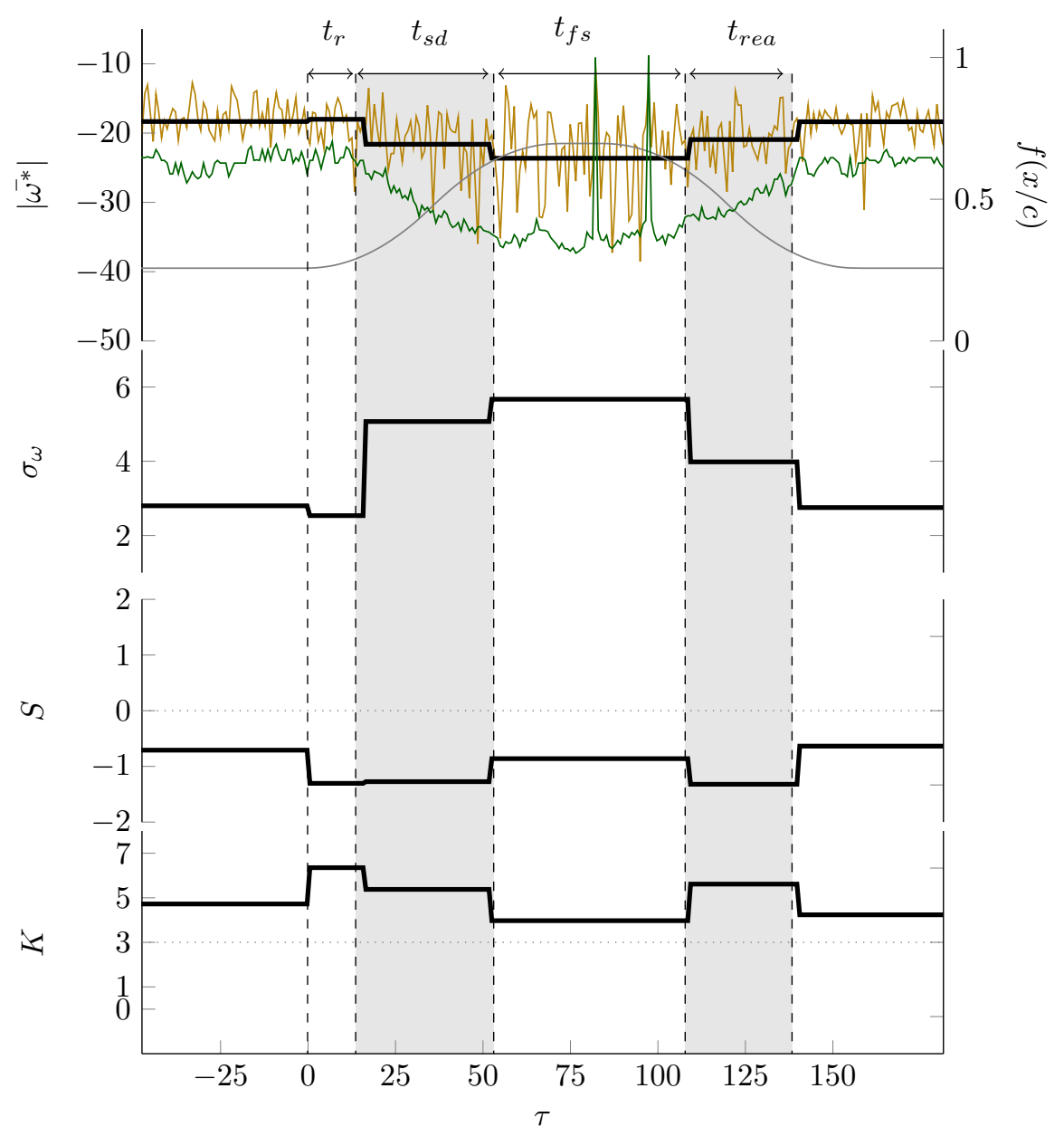

Figure 7.2: Peak vorticity at the point of separation (gold) plotted as a function of $\tau$, plotted along side the separation point (green) and the motion profile (grey), with the mean, standard deviation, skewness, $S$, and kurtosis, $K$, plotted as functions of time for the selected stall stage at $z / R=0.187$ at a pitch rate of $\dot{\alpha}=34.28$.

is a DU 350 profile. Figure 7.3 depicts the peak vorticity in the same format as figure 7.1. An initial mean value of $\left|\omega^{*}\right|=21.7$ in encountered with a standard deviation of $\sigma_{\omega}=2.9$. Unlike the previous plane where the stall developed in a more gradual manner, the separation point in figure 7.3 approaches the leading edge in a stepwise form. As seen previously, the mean peak vorticity reaches a 


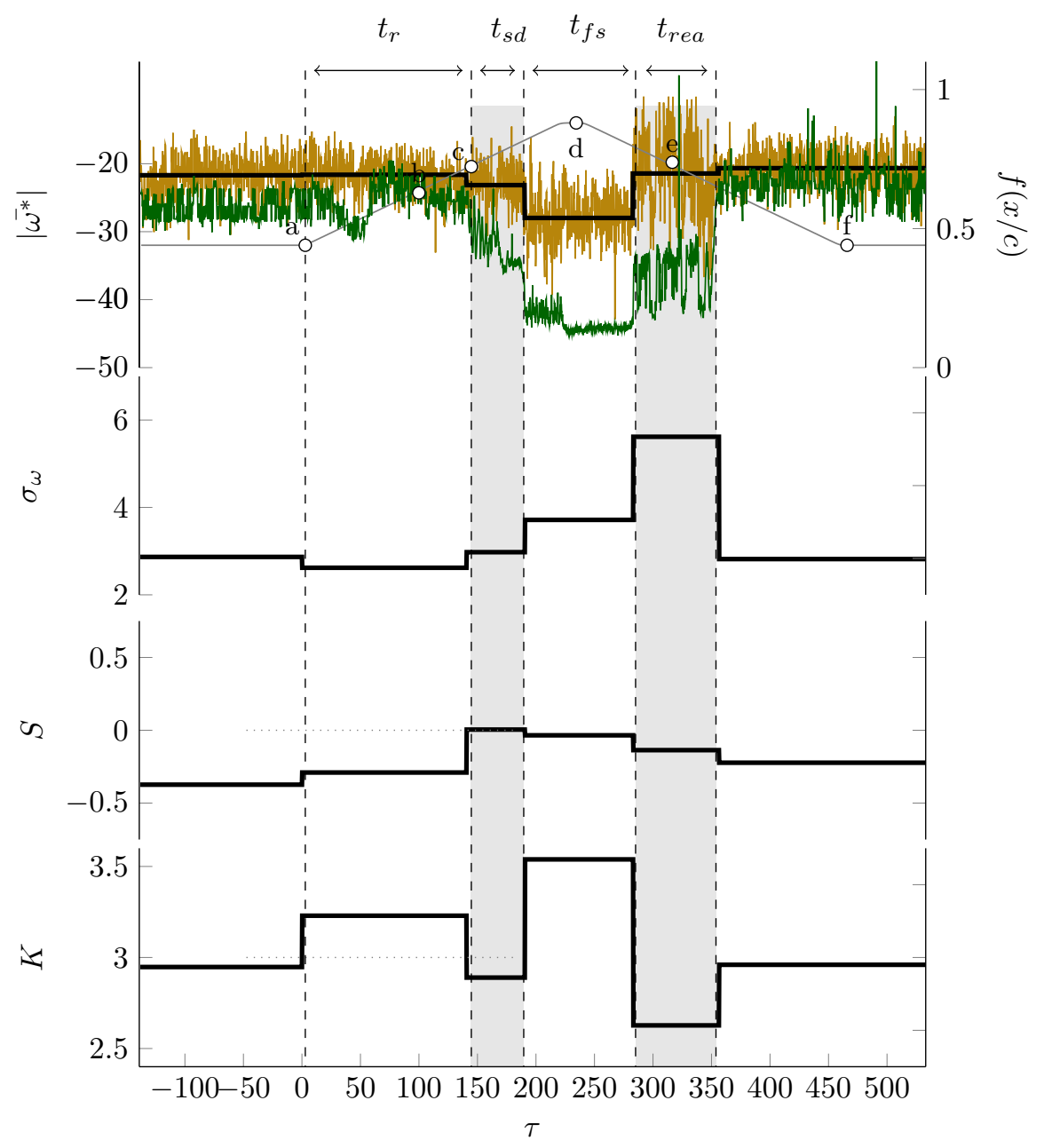

Figure 7.3: Peak vorticity at the point of separation (gold) plotted as a function of $\tau$, plotted along side the separation point (green) and the motion profile (grey), with the mean, standard deviation, skewness, $S$, and kurtosis, $K$, plotted as functions of time for the selected stall stage at $z / R=0.252$ at a pitch rate of $\dot{\alpha}=17.14$.

maximum during full stall, here $\left|\omega^{*}\right|=27.9 \pm 3.7$, a $29 \%$ increase from its attached flow value. Unlike the inner most plan, $\sigma_{\omega}$ reaches a maximum, not during full stall, but rather during reattachment. The skewness of the peak vorticity, begins negative and reaches nearly zero during stall development. Kurtosis changes from excess to 


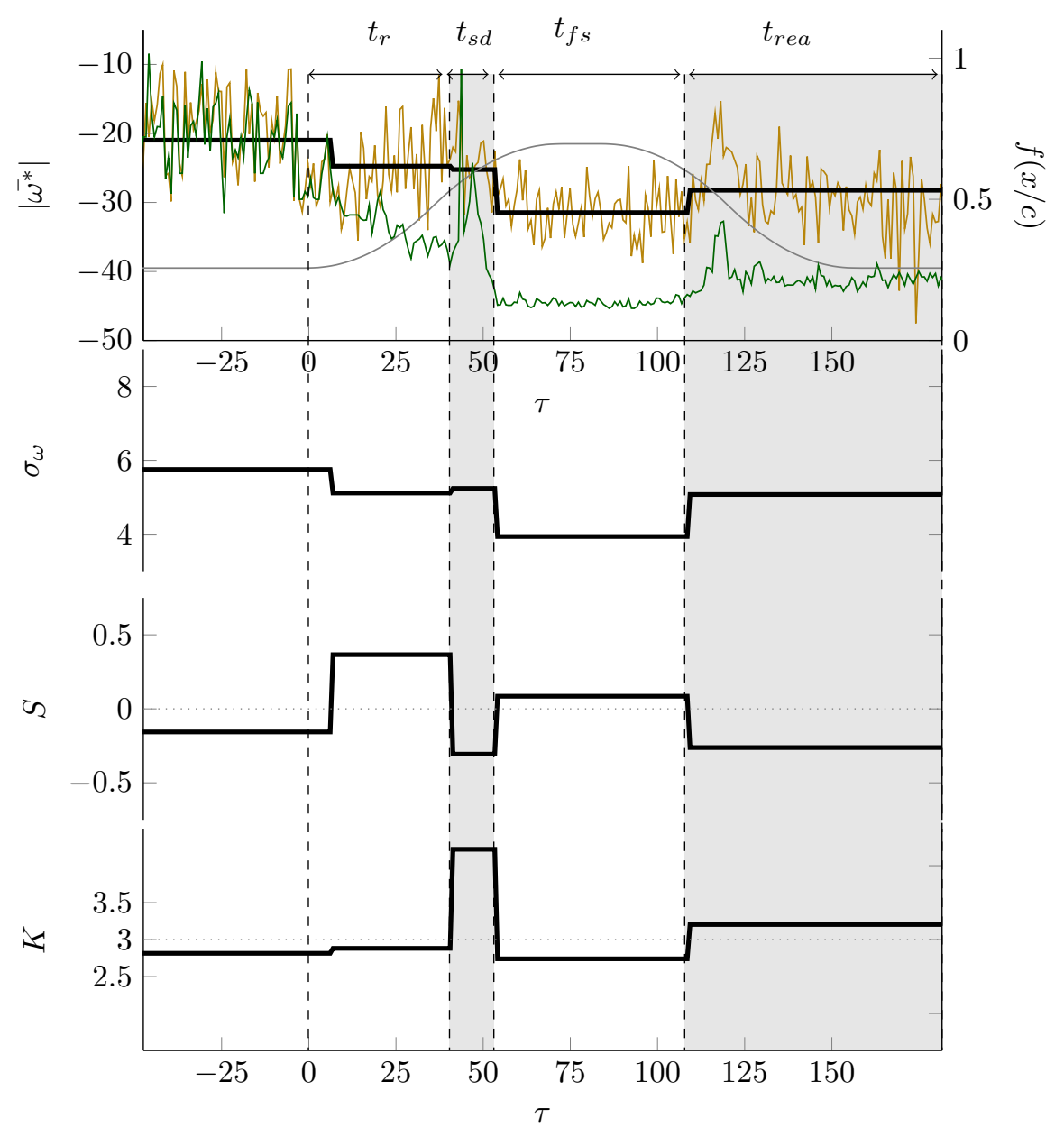

Figure 7.4: Peak vorticity at the point of separation (gold) plotted as a function of $\tau$, plotted along side the separation point (green) and the motion profile (grey), with the mean, standard deviation, skewness, $S$, and kurtosis, $K$, plotted as functions of time for the selected stall stage at $r / R=0.252$ at a pitch rate of $\dot{\alpha}=34.28$.

deficit during the transitions with a maxima in full stall. The DU 350 profile, when subjected to a faster pitch rate and is presented in figure 7.4, begins with a less stable initial condition. This instability leads to a relatively short reaction time, and a more gradual separation process than what was seen in the slower pitching sequence. Despite the unsteady behavior, the mean peak vorticity is nearly identical 
to the slower counter part, with $\left|\omega^{*}\right|=20.9$. However, due to the highly unsteady behavior of the separation point, the vorticity peak in this case has a standard deviation of $\sigma_{\omega}=5.75$. The stall development stage is considerably shorter and is characterized by a substantial jump in the separation point towards the trailing edge before finally reaching full stall. The jump traverses over $68 \%$ of the chord within $4 \tau$ when the angle of attack reaches $\alpha=23.9^{\circ}$. Once in full stall, the mean vorticity peak reaches a mean value of $\left|\omega^{*}\right|=31.5$ and over all increase of $50 \%$ and the standard deviation drops by $32 \%$ to $\sigma_{\omega}=3.9$. In this case, the reattachment stage begins at $\alpha=23.1^{\circ}$. However, the flow does not reattach during the remaining portion of the measurement series.

The separation point in figure 7.5 contains a gradual approach to the leading edge as stall begins. Once the stall development stage starts, there is a brief moment of reattachment at $\tau=150$, during which time the separation point recedes from the leading edge by nearly 20\%. Full stall occurs along this cross section when the blade reaches an angle of attack of $\alpha=23.4$. Reattachment start suddenly as the angle of attack drops below $\alpha=24^{\circ}$ and gradually returns to its initial position of $f(x / c)=0.6$ when the angle of attack reaches $\alpha=19.5^{\circ}$. The vorticity peak for this measurement series shows large oscillations about the mean throughout the entire stall cycle, but the mean does not show a significant increase when the flow enter into full stall. The mean which begins at $\left|\omega^{*}\right|=25$ during attached flow increases nominally to $\left|\omega^{*}\right|=26$ in full stall. Unlike any of the previous data sets, the transitions show the smallest average peak vorticity across the stall stages finding a minimum value of $\left|\omega^{*}\right|=24$ during stall development. The standard deviation is the of the peak vorticity, increases throughout the stall cycle starting at $\sigma_{\omega}=6$ 


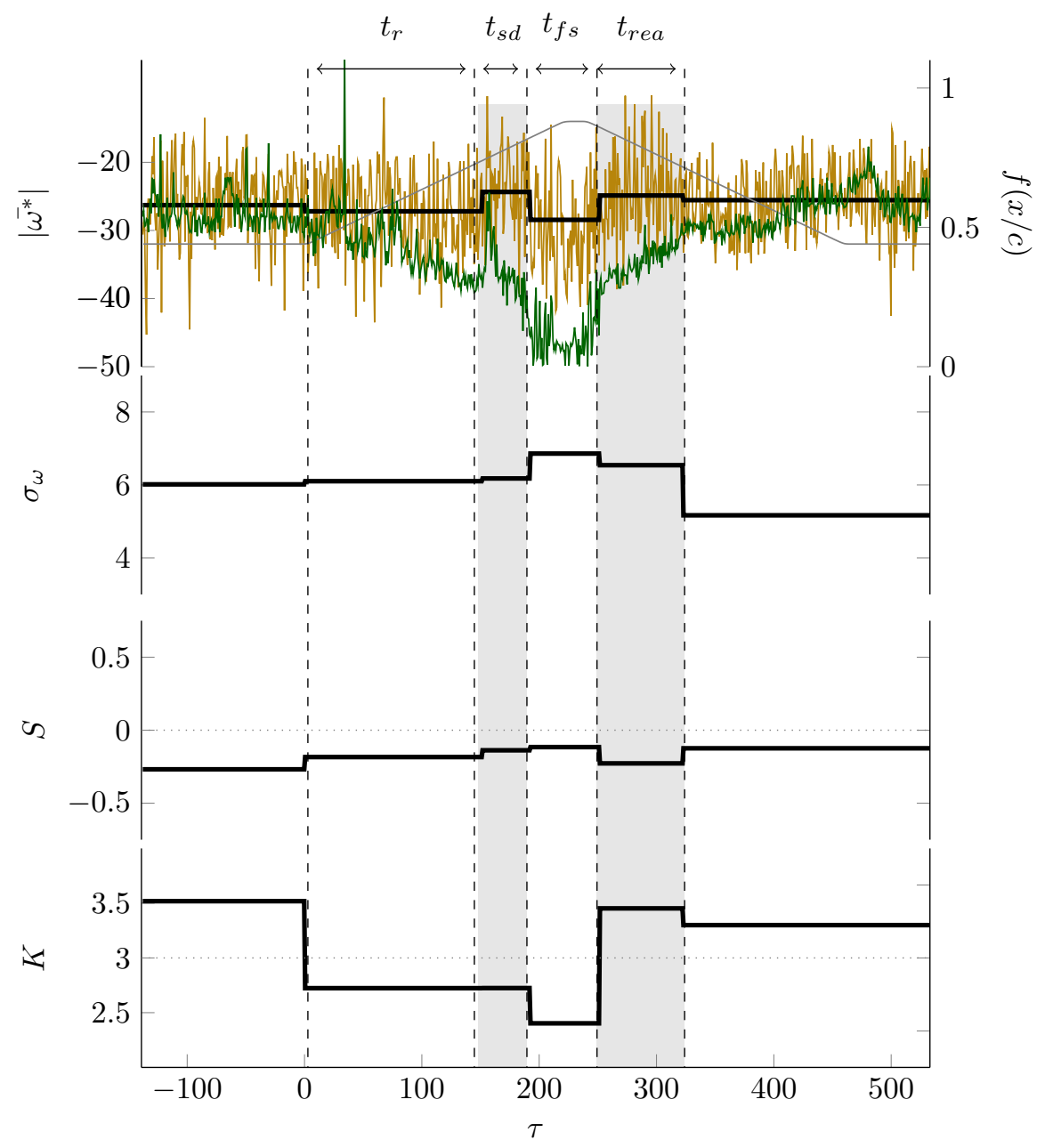

Figure 7.5: Peak vorticity at the point of separation (gold) plotted as a function of $\tau$, plotted along side the separation point (green) and the motion profile (grey), with the mean, standard deviation, skewness, $S$, and kurtosis, $K$, plotted as functions of time for the selected stall stage at $r / R=0.317$ at a pitch rate of $\dot{\alpha}=17.14$.

during attached flow, increasing slightly during full stall to $\sigma_{\omega}=7$ before reaching its minimum after flow reattaches at $\sigma_{\omega}=3$.

The skewness, $S$, for the DU 350 profile at $r / R=0.317$ subjected to a pitch rate of $\dot{\alpha}=17.14$, remains negative throughout the stall cycle, which follows a 


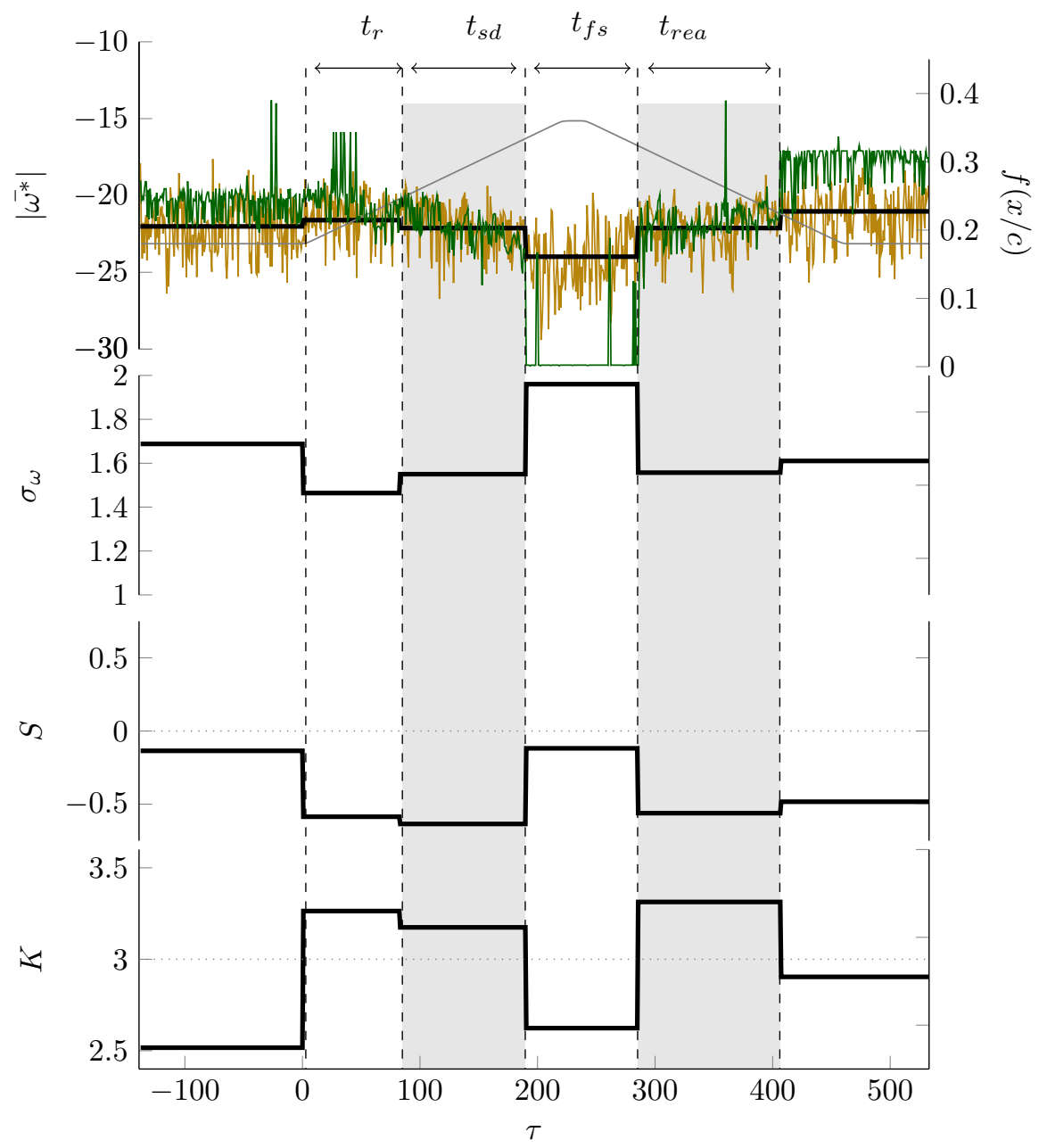

Figure 7.6: Peak vorticity at the point of separation (gold) plotted as a function of $\tau$, plotted along side the separation point (green) and the motion profile (grey), with the mean, standard deviation, skewness, $S$, and kurtosis, $K$, plotted as functions of time for the selected stall stage at $r / R=0.382$ at a pitch rate of $\dot{\alpha}=17.14$.

similar trend to the other slower pitching cycle that has been discussed previously. The kurtosis during the stall cycle reaches a minimum at full stall and is maximum during attached flow, an indication of steady vorticity shedding during full stall and less so during the other stages. 
At the mid span position of $r / R=0.38$, shown in figure 7.6, the initial separation point begins at $f(x / c)=0.2$. This is due to the stationary angle of attack, which is in excess of $\alpha=18^{\circ}$. The overall movement of the separation point is gradual as the blade is pitched up until the blade enters into full stall, at an angle of attack of $28^{\circ}$. The reattachment passes through two stages, with the separation point dropping to $f(x / c)=0.2$ at $\alpha=18.55^{\circ}$.

The peak vorticity for this plane shows a slight increase throughout the stall cycle from $\left|\omega^{*}\right|=22$ during attached flow and reaching $\left|\omega^{*}\right|=25$ during full stall. The standard deviation is relatively small, on the order of $\sigma=1.5$ throughout, reaching a maximum during full stall of $\sigma=1.8$.

The skewness of the vorticity peak is consistently negative which holds with the previous examined slower pitching regimes, showing lower magnitudes during attached flow and full stall. Meanwhile, the kurtosis passes from a deficit during the aforementioned stages into excess during transitions. When influenced by a higher pitch rate, figure 7.7 , the profile at $r / R=0.38$ shows a similar separation point behavior to the vorticity peak statistics, leading to the conclusion that due to the excessive stall behavior present prior to the pitching cycle, the stall characteristics do not show sensitivity to a changing pitch rate.

Results for the inboard plane at $r / R=0.187$ show that the vorticity dynamics of the first plane are relatively consistent between the pitch rates. The transition to stall is gradual, relative to the thinner cross-sections. The thickness of the airfoil also causing a gradual stall cycle, which is a sign of aerodynamic stability within this region.

In summary, it is noted that the inboard and outboard profiles are less sensitive 


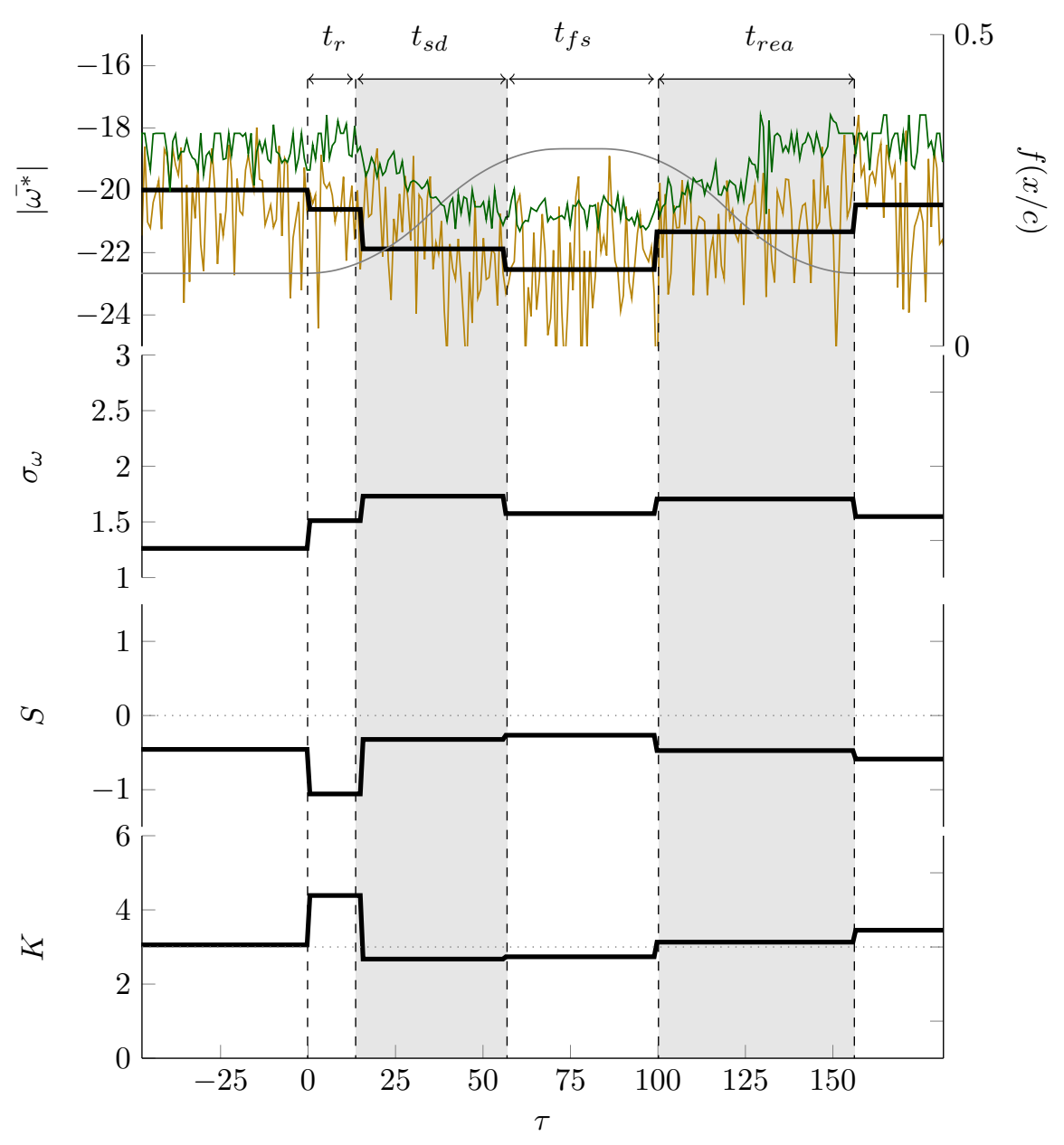

Figure 7.7: Peak vorticity at the point of separation (gold) plotted as a function of $\tau$, plotted along side the separation point (green) and the motion profile (grey), with the mean, standard deviation, skewness, $S$, and kurtosis, $K$, plotted as functions of time for the selected stall stage at $r / R=0.382$ at a pitch rate of $\dot{\alpha}=34.28$.

to $\dot{\alpha}$ than the middle two planes. Both the inboard and out board planes have gradual transitions and experience moderate increases in the surface vorticity as the flow transitions into full stall. Meanwhile, the internal planes at $r / R=0.252$ and $r / R=0.317$ show stepwise transitions. The separation point, $f(x)$, fluctuates around quasi-static chordwise positions during each transitional stall stage. These 
planes also show slightly higher separation point vorticity than their counterparts.

When comparing the surface vorticity between the two pitch rates, the peak vorticity is consistently higher when the pitch rate is increased. The other key finding is that for each of the lower pitching rates, the vorticity peak is skewed negatively, indication that the build-up of vorticity is gradual and tends to exceed the mean briefly during the stall cycle before the surface vortex is shed and the peak drops once again below the mean. However, when these profiles experience a higher pitch rate, the building vorticity is able to stay near the surface longer during transitions. Persistence of the surface vorticity leads to the peak vorticity exceeding the mean more often.

\subsection{Peak vorticity span and speed comparison}

Evaluating over all interrogation areas the spanwise peak vorticity development. Figure 7.8 depicts the distribution of the separation point vorticity for the slower pitch rate in solid color. The fast pitch rates presented with slanted lines, for each of the four measurement planes. The distribution of vorticity peaks at the point of separation is represented as a percentage of measured values throughout each experiment.

In figure 7.8, each of the distributions are centered over a vorticity peak between $-25 \leq \omega^{*} \leq-20$. For three of the four planes, namely $r / R=0.19,0.25,0.38$, this range is the most common. A range that accounts for over $40 \%$ of the measured vorticity peaks. At $r / R=0.38$, this range of vorticity is present over $65 \%$ of the measurement series. At $r / R=0.38$, the distribution is quite narrow relative to the other planes, where over $90 \%$ of the separation vorticity is found between $-25 \leq$ 
$\omega^{*} \leq-30$. The inner two planes distributions are relatively flatter with $90 \%$ of peak vorticity falling between $-15 \leq \omega^{*} \leq-35$. Peak vorticity at $r / R=0.19$, is normally distributed with a mean falling between $-25 \leq \omega^{*} \leq-20$. A normal distribution shows that this plane, which possesses the thickest profile, separates gradually with regular build up and shedding of vorticity relative to the thinner profiles along the span. The profile at $r / R=0.32$ has flattest the distribution, generating peak vorticity exceeding $\omega^{*}=30$ in over $20 \%$ of the experiment. Consequently, this plane proves to vary the most in its separation behavior.

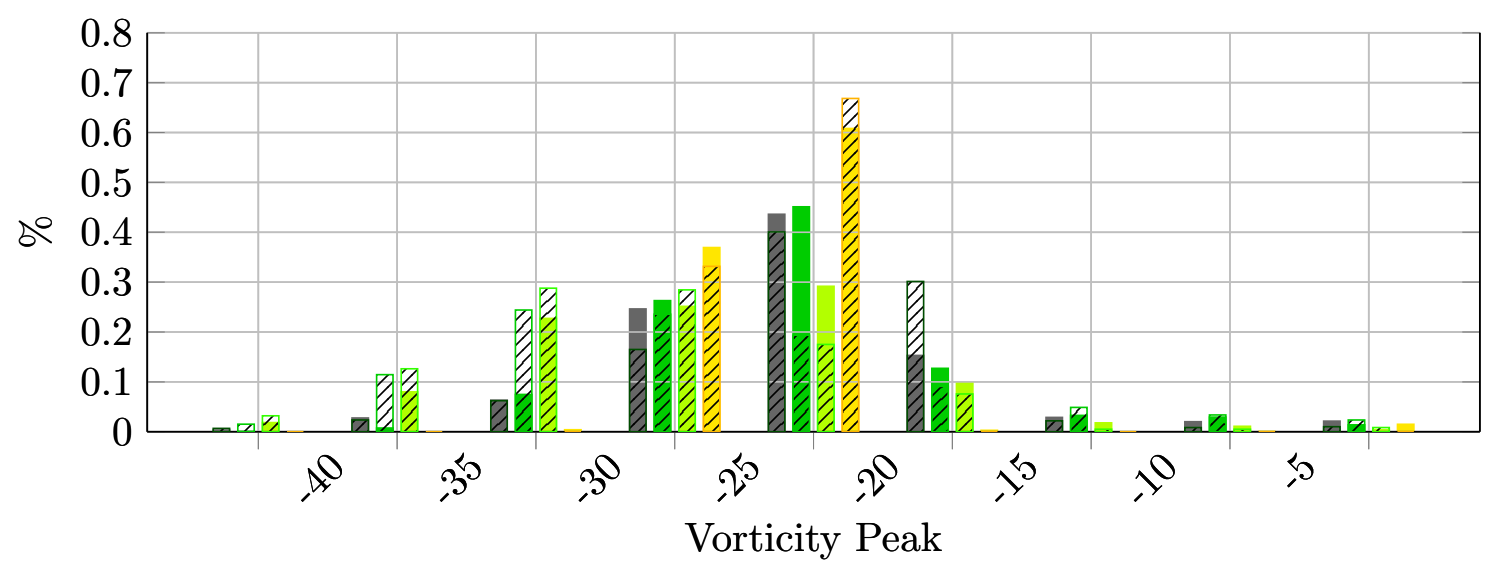

$$
\| z / R=0.19 \square z / R=0.25 \square z / R=0.32 \square z / R=0.38
$$

Figure 7.8: Separation point peak vorticity histogram for all measurement planes, $r / R=0.19,0.25,0.32$, and 0.38 for pitching rates, $\dot{\alpha}=17.14$ (solid) and 34.28 (dashed), with magnitude $\left(\omega^{*}\right)$ on the $x$-axis and frequency of occurrence (\%) on the $y$-axis.

When subject to a fast pitch rate, figure 7.8, the separation vorticity distributions shift. The distribution of peak vorticity at $r / R=0.19$ and $r / R=0.38$ shift to lower magnitudes. At $r / R=0.19$, the mean drops to fall in between $-20 \leq \omega^{*} \leq-15$, and is no longer Gaussian. The distribution is skewed positively, with more excursions 
into higher vorticity peaks. At the mid-span position of $r / R=0.39$, its mean remains within the $-25 \leq \omega^{*} \leq-20$ range. However, the frequency at which the peak occurs increases. The shift in the two middle planes exalts towards higher peak vorticity at separation. At $r / R=0.252$, the skew of the peak vorticity has changed signs, with the tail of the distribution longer on the side of lower peak magnitudes. The vorticity peak distribution at $r / R=0.32$ are flatter with a mean that has increased from $\approx 23$ to $\approx 30$.

Notable observations are that when the pitch rate increases, the peak vorticity generated at the point of separation is higher for the middle two cross sections. This is attributed to the rate of vorticity build-up exceeding the rate of shedding due to the addition of the negative surface pressure generated by a higher pitching rate. The furthest inboard and the outer most cross-sections do not show the same sensitivity to the pitch rate, which is attributed to the relative thickness and gradual separation behavior of the inboard section. Despite those differences, the histograms also reveal that the separation point vorticity is remarkably consistent from plane to plane. This quantity, as it applies to the unsteady separation over this airfoil, is taken as a critical value pertaining to the emergence and persistence of such phenomena.

\subsection{Comparison of spanwise and pitch rate dependence of POD coeffi- cients}

As outlined in chapter 4, the application of proper orthogonal decomposition to a PIV measurement field during unsteady separation allows for the evaluation of distinct time scales for each of the energetic eigenmodes which contribute to the 
various stall stages. As previously shown, the first eigenmode describes the highest energy state for the measurement series, representing the attached flow state. Thus, the associated time coefficient, $a_{1}$ is used to determine the period of time where the flow remains attached. The second eigenmode represents the period of full stall and its time scale is extracted using the time coefficient, $a_{2}$. The third eigenmode is taken to represent the the transitional stages of stall development and reattachment, and their respective times scales are captured via $a_{3}$. It follows that when each time coefficient is maximized, the flow is considered to be under the influence of that eigenmode, and thus experiences the corresponding flow state. For example, when $a_{1}$ is at its maximum value, the flow is attached.

To compare the dynamics of these relationships, the coefficients for $r / R=0.252$ at $\dot{\alpha}=34.28$ are plotted against on another in figures $7.9(\mathrm{a})$ and $7.9(\mathrm{~b})$. In figure $7.9(\mathrm{a}), a_{1}$ is plotted on the $x$-axis and $a_{2}$ on the $y$-axis and in figure $7.9(\mathrm{~b}) a_{2}$ is plotted on the $x$-axis and $a_{3}$ on the $y$-axis, presenting attached flow in yellow and full stall in green.

The results shown in figure 7.9(a) during attached flow, $a_{1}$ is at its largest magnitude and $a_{2}$ is at its minimum. Then as the pitching motion begins, the balance between the two time coefficients evolves as the flow transitions to stall, with only slight preferences toward the eigenmode that represents the stage that the flow is closest to. Once the flow enters full stall, the second eigenmode is the dominant contributor between the two. During which time, the first coefficient is greatly reduced.

In figure 7.9(b), the dynamic between the second and third eigenmodes is noticeably different than the relationship between the first and second mode. In this 


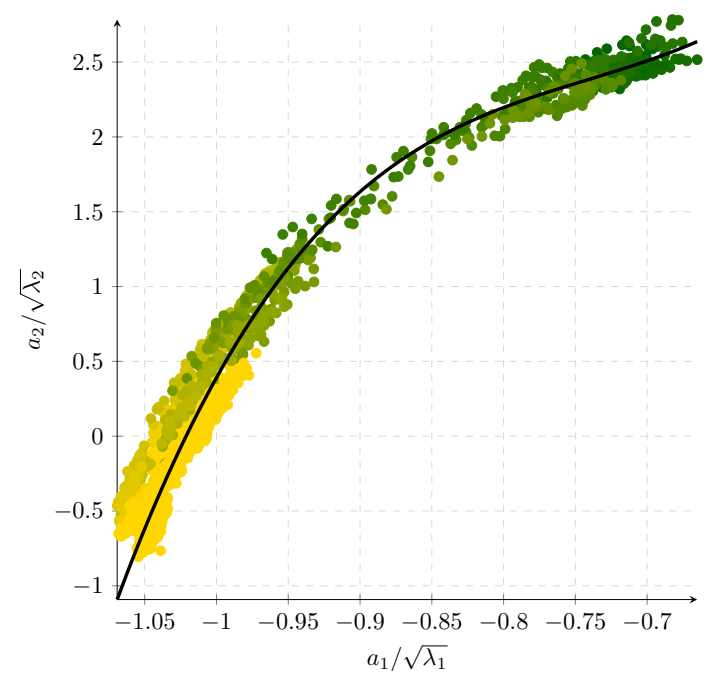

(a) $a_{1}$ vs. $a_{2}$

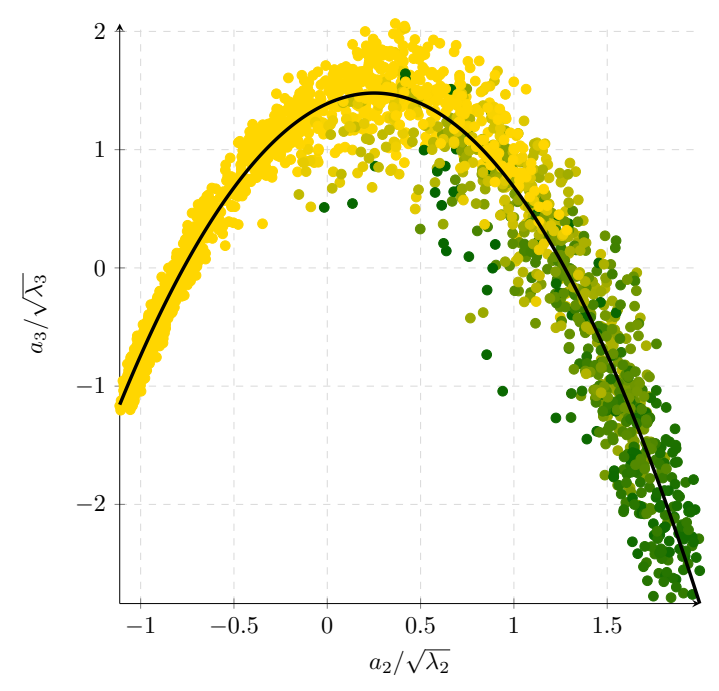

(b) $a_{2}$ vs. $a_{3}$

Figure 7.9: Normailzed POD time coefficients (a) $a_{1} / \sqrt{\lambda_{1}}$ vs. $a_{2} \sqrt{\lambda_{2}}$ and (b) $a_{2} \sqrt{\lambda_{2}}$ vs. $a_{3} \sqrt{\lambda_{3}}$, plotted with color scheme that highlights attached flow in yellow and full stall in green for $r / R=0.252$ at $\dot{\alpha}=34.28$.

case, both modes are contributing equal parts to the attached flow state. However, as the motion begins and stall develops, it is mode 3 that grows significantly faster during transition. As flow begins to approach full stall, the magnitude drops and the second mode grows. Both coefficients undergo a sign change and arrive at nearly equal magnitudes at the full stall state.

Extending this comparison to each of the planes and pitching rates, the trend lines for $a_{1}$ and $a_{2}$ each are plotted in figure 7.10(a) and $a_{2}$ and $a_{3}$ in figure 7.10(b) with $\dot{\alpha}=17.14$ in solid lines and $\dot{\alpha}=34.28$ represented with dashed lines.

Remarkably, the relationship between the various eigenmodes show consistency from case to case. Although there are notable differences in the rates at which one mode contributes relative to one another, the general behavior is such that in each figure, 


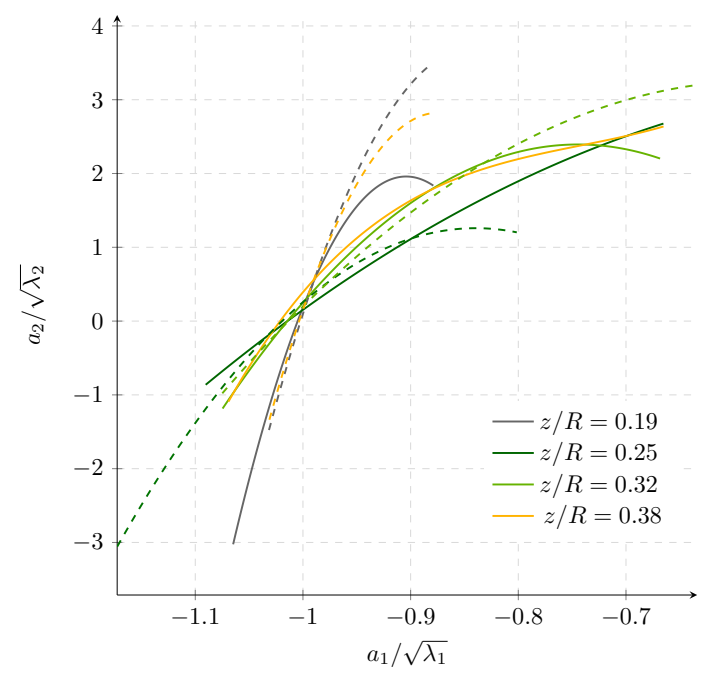

(a) $a_{1}$ vs. $a_{2}$

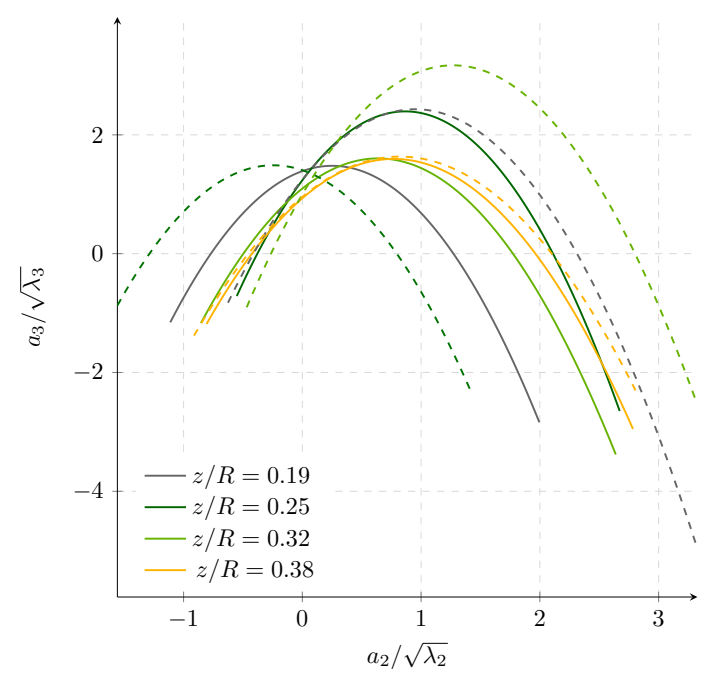

(b) $a_{2}$ vs. $a_{3}$

Figure 7.10: Normailzed POD time coefficients (a) $a_{1} / \sqrt{\lambda_{1}}$ vs. $a_{2} \sqrt{\lambda_{2}}$ and (b) $a_{2} \sqrt{\lambda_{2}}$ vs. $a_{3} \sqrt{\lambda_{3}}$, plotted with color scheme that highlights attached flow in yellow and full stall in green for $r / R=0.190 .38$ at $\dot{\alpha}=17.14$ (solid) and 34.28 (dashed)

despite the spanwise position or pitch rate all trends cross each other at the same point. In figure $7.10(\mathrm{a})$ all of the trend lines meet when $a_{1}=-1$ and $a_{2}=0$. In figure 7.10(b) the meeting point occurs when $a_{2}=0$ and $a_{3}=1$. For both sets, the trend lines meet when $a_{2}=0$. At this point in their respective time series, the flow is transitioning into stall at one crossing and reattaching during the other. The resilience of the general trend speaks to the independence of POD to the external influence of pitch rate and spanwise position. When comparing the dependence upon pitch rate, in general the slower pitch rate yields a lower slope for the coefficient relations, but the only plane that shows a considerable change is at $r / R=0.38$. The remaining planes show only slight changes due to pitch rate. The main difference in the relationship between $a_{1}$ and $a_{2}$ is seen when moving from plane to plane. The 
rate of change between the coefficients is largest at the inboard and outboard planes, where transitions were were predominantly gradual when compared to the planes at $r / R=0.25$ and 0.32 . This spanwise dependence shows that when the transitions progress gradually, the influence of the first mode persists into deeper stall stages.

Physically, the stall state is passing between fully separated and fully attached if the transition is gradual. In the relationship between the second and third modes, the relationship does not appear to vary much with pitch rate or even from plane to plane. The only difference between the two being the magnitude of the eigenmodes.

\subsection{Separation point quantities comparison}

The study of unsteady separation is based on the uncertainty of where and when separation will occur over a surface. The distance between the origin of the boundary layer and the point of its eventual separation from the surface is a critical length scale in predicting aerodynamic loads. Figures 7.11(a) and 7.11(b) present a calculation of this distance as it varies with stall extent based on the slower and faster pitching rates, respectively. The figures give the boundary layer origin, $S e p_{u}$, along the surface, determined by the emergence of the shear layer velocity, $u_{s}$. The first occurrence of the shear-layer velocity, defined as $u_{s}=0.48 U_{\infty}$, is plotted along the $x$-axis and the separation point $S e p_{\omega}$, which is captured by the first occurrence of zero vorticity, is plotted along the surface of the airfoil on the $y$-axis, following the MRS criterion. The color map of these plots targets the period of full stall, represented by dark green, and the attached flow data points are plotted in yellow. For reference, a black line is plotted which represents the points where these two points along the surface equate to each other. 
Figure 7.11(a) compares these two quantities for all interrogation areas subject to the slower of the two pitching regimes. The trend displays the variance between these two values being constant at its minimum distance; varying rarely by less than $5 \%$ of the chord. Consequently meaning that the boundary layer is rarely shorter than 0.05c. The variation between the two quantities decreases as the point of separation approaches the leading edge, reaching a minimum when the cross-sections are in full stall. During which time the difference between the two separation points is no larger than 0.1c. This gives the maximum distance that the shear layer can travel before separating during full stall. The variance is maximized at the beginning and end of the measurements during attached flow, where the difference between these can be over $50 \%$ of the chord. Meaning that the boundary layer is able to form and remain near the surface over a longer distance before recirculation occurs. The variance of $S e p_{\omega}$ and $S e p_{u}$ passes through two linear regimes: The first is located when separation is near to the leading edge and the mid-chord, where the difference grows with a slope of $\approx 1$, and the second occurs once the separation point retreats beyond $x / c=0.5$. When flow is attached to the surface, the difference grows and the slope increases. When the flow is in this state, the minimum difference has increased to $10 \%$.

The trend in figure 7.11(b), which represents the measurements conducted at a faster pitch rate, shows a similar trend to the slower pitch. Here, a convergence between the separation point values as they approach the leading edge is noted. When crossing from attached flow into full stall, at $\approx x / c=0.3$, the difference increases significantly to where the zero vorticity may or may not be pushed further towards the trailing edge. This is taken as a manifestation of the primary stall 


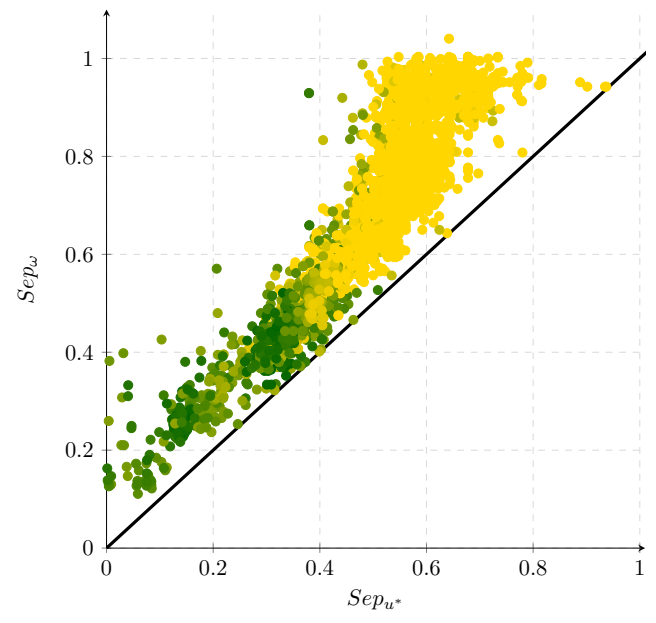

(a) $\dot{\alpha}=17.14$

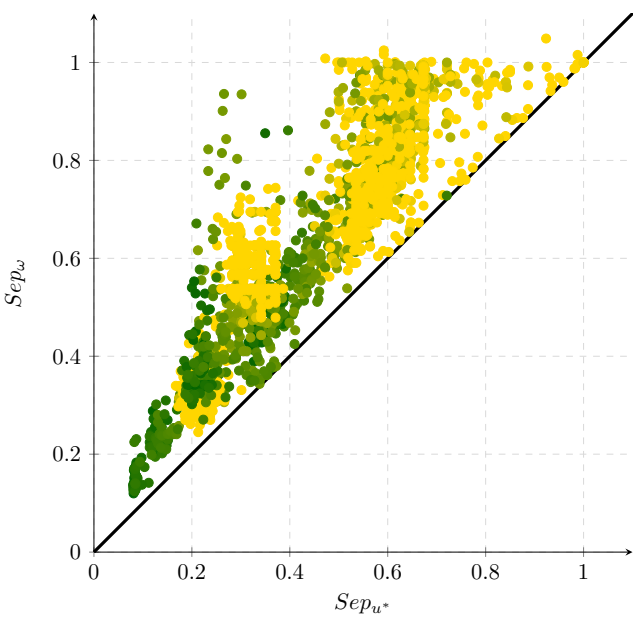

(b) $\dot{\alpha}=34.28$

Figure 7.11: Shear layer origin based on the convective velocity of $0.48 U_{\infty}$ plotted against the fist occurrence of zero vorticity within the separated volume for all planes under the influence of a pitch rate of $(a) \dot{\alpha}=17.14$ and $(b)$ $\dot{\alpha}=17.14$, tracking from attached flow stages (yellow) to full stall (green).

vortex which has been noted previously to draw the shear layer towards the surface. This behavior, due to generated negative pressure along the surface, is responsible for reattaching flow during stall development. This phenomena was absent in figure 7.11(a), an indication that due to the faster pitch rate, the persistence of surface vorticity during transitions can drive the $S e p_{\omega}$ to the trailing edge. During these times distance between $S e p_{\omega}$ and $S e p_{u}$ can be as large as $60 \%$ of the chord.

Within these two plots, the difficulty of predicting where flow will separate in unsteady conditions is highlighted by the disparity between the two separation criterion. Specifically during transitions, where the unsteady behavior caused by increasingly faster changes in the angle of attack can drive the vorticity criterion down to the trailing edge. 


\subsection{Amplitude ratios}

The development of unsteady separation is also connected to the ampltidues of shear layer perturbations, $A$. As the perturbations grow and approach the point of separation, it can lead directly to the movement of the separation point itself [26]. Figures 7.14 and 7.13 and aim to quantify the relationship between the growth of the perturbations with respect to the point of separation. Figure 7.14 demonstrates how these quantities are determined by plotting the ratio between the first perturbation amplitude $A_{1}$ and the maximum shear layer perturbation $A_{\max }$ along the $x$-axis and the maximum amplitude on the $y$-axis. These values are taken at their absolute values for clarity. The color scheme is used to define the stall stages, with yellow representing attached flow and green representing full stall. In figure 7.14 the slow pitch rate at $r / R=0.252$ is presented. A least squares fit is taken to determine the trend. Following the trend of the ratio being typically a minimum when the amplitudes of the shear layer grows, the growth rate of the perturbations owing to Kelvin-Helmholtz instability is captured. The maximum perturbations occur when the airfoil is in full stall, however the data points break the trend and encroach larger amplitude ratios are found in the transitions, presented in light green. The largest ratios are found predominantly when the maximum amplitude is relatively small. Occasionally, these two values are the same, but this only occurs when the airfoil is in an attached flow regime.

In figure 7.13 , this technique is applied to all measurements and divided in to slow and fast pitching regimes, respectively. To begin, the slower pitching regime is taken to represent a gradual stall development over an extended gust. The amplitude ratio has three important areas for comparison, first is the maximum amplitude reached 


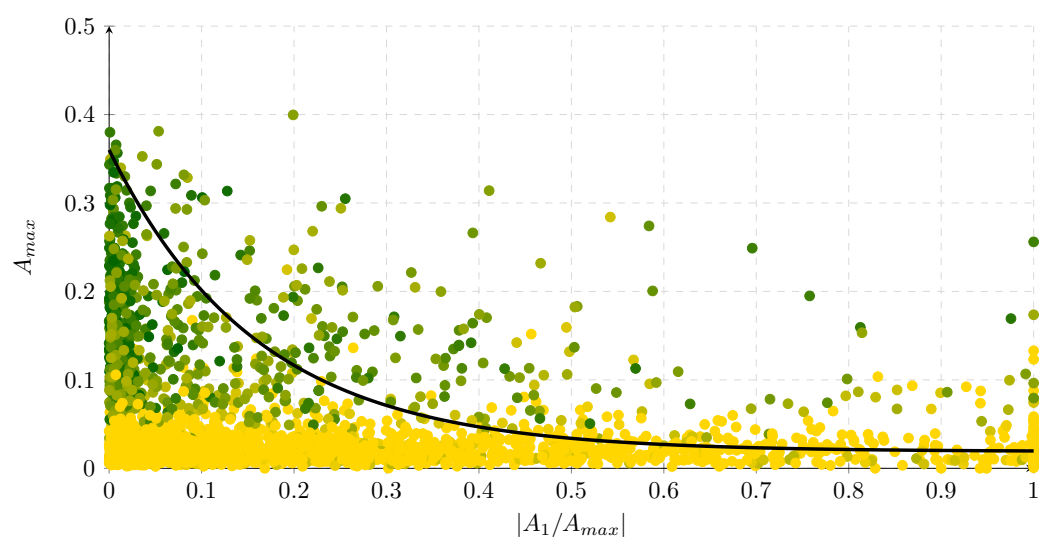

Figure 7.12: Ratio of first shear layer perturbation amplitude $A_{1}$ and maximum amplitude $A_{\max }$ on the $x$-axis plotted against $A_{\max }$ on the $y$-axis, with stall stages mapped from attached flow states (yellow) to full stall (green).

in each plane, second is the rate of incline or the hip least squares fit, and last is the constant offset when the ratio is maximum. For the spanwise comparison of the maximum amplitudes, it is $r / R=0.32$ that stands out, reaching a maximum amplitude of $0.6 c$ during full stall. The next lowest maxima are found at $r / R=0.25$ and $r / R=0.38$ with a maximum of $0.35 c$ and $0.3 c$, respectively. The full stall amplitudes of the inboard plane are by far the smallest, only reaching $0.2 c$.

When comparing the growth rate or the hips of the least squares fit, $r / R=0.25$ and $r / R=0.32$ have very similar growth rates as stall develops running in parallel as the amplitude ratio approaches its minimum from nearly constant maximum amplitudes at amplitude ratios reach 0.6 and 0.8 respectively. The growth rate of $r / R=0.38$ is lower then at $r / R=0.25$ and 0.32 , starting when the amplitude ratio decreases below 0.4 and the lowest growth rate is found to be at the inboard plane, where the maximum amplitude grows almost linearly through the stall cycle.

The constant offset represents the steady state of the experiment. It shows that 
at $r / R=0.19$ and 0.25 a standing wave exists that has a maximum amplitude of $0.03 c$. With values at $r / R=0.25$ being slightly smaller than at $r / R=0.19$, the outer two planes, which generally experience a larger stall region during steady state, have steady amplitudes of $0.07 c$ and $0.1 c$.

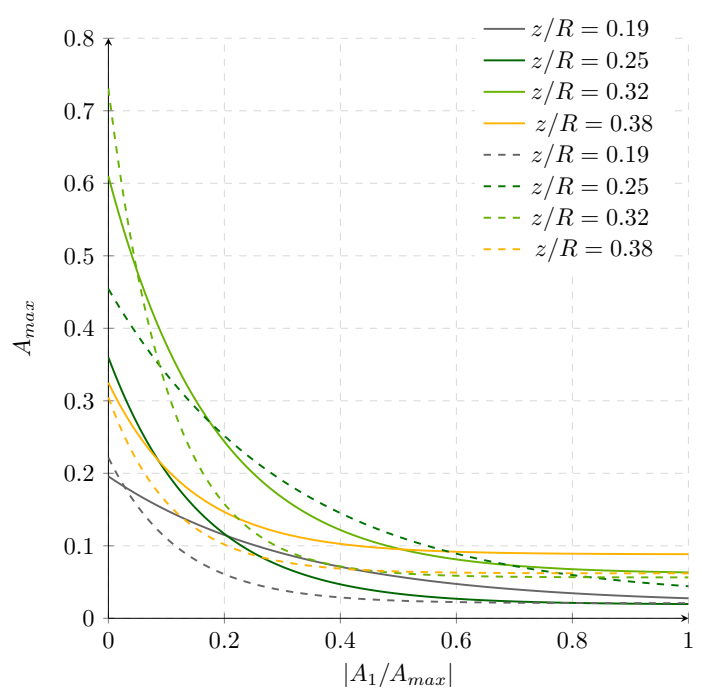

Figure 7.13: Ratio of first shear layer perturbation amplitude $A_{1}$ and maximum amplitude $A_{\max }$ on the $x$-axis plotted against $A_{\max }$ on the $y$-axis, with stall stages mapped from attached flow states (yellow) to full stall (green).

When exposed to a faster pitch rate, the growth rates show a marked increase across the span. The only exception occurs at $r / R=0.25$, where growth rate has decreased. This is attributed to the fact that the flow does not reattach and thus there are significantly larger amplitudes within the data set. Once again the global maximum amplitude is found at $r / R=0.32$, indication that this plane is the most unstable during stall development. Amplitudes at $r / R=0.25$ maintain the second position with magnitudes of over $0.4 c$ followed by $r / R=0.38$ and $r / R=0.19$ respectively holding their rank along the span. However, the maximum amplitude 
for $r / R=0.25$ has grown from $0.3 c$ to $0.45 c$ when exposed to a faster pitch rate. While the maximum amplitudes for $r / R=0.19$ and $r / R=0.38$ have not changed between pitch rates, the rate of perturbation growth is higher for both planes, with the inboard plane showing the most distinct increase.

In summary, across all planes and pitch rates, the maximum perturbations occur when the airfoil is in full stall. Conversely, the largest ratios are found when flow is attached. Physically, this shows that the perturbations grow as the stalled region expands. There is a constant offset along the $y$-axis which reveals the amplitude is constrained when flow is attached. The spanwise dependence of the maximum amplitude shows that for relatively thinner profiles, the amplitudes and their growth rates can be significantly larger than for the thicker profiles. Leading to the conclusion that a thinner profile undergoes stall process that is highly unsteady relative a thicker blade.

\subsection{Amplitude variation with separation point}

The dynamics of shear layer perturbations, A, based on the separation point, $f(x / c)_{\omega=0}$, defined by zero vorticity, are captured in figure 7.14. The global perturbation maximum and minimum from each shear layer measurement are plotted on the $y$-axis against separation point on the $x$-axis. For temporal reference, the color map presents the attached flow regimes in yellow and the full stall regime in dark green. Once again, a least squares fit is applied, to generate the spanwise and pitch rate dependence of generated shear layer amplitudes.

Shear layer maxima and minima on the $y$-axis plotted against the separation point $f(x / c)_{\omega=0}$ on the $x$-axis, with stall cycle following from attached flow (yellow) 
for full stall (green) at a pitch rate $\dot{\alpha}=17.14$ at $r / R=0.32$.

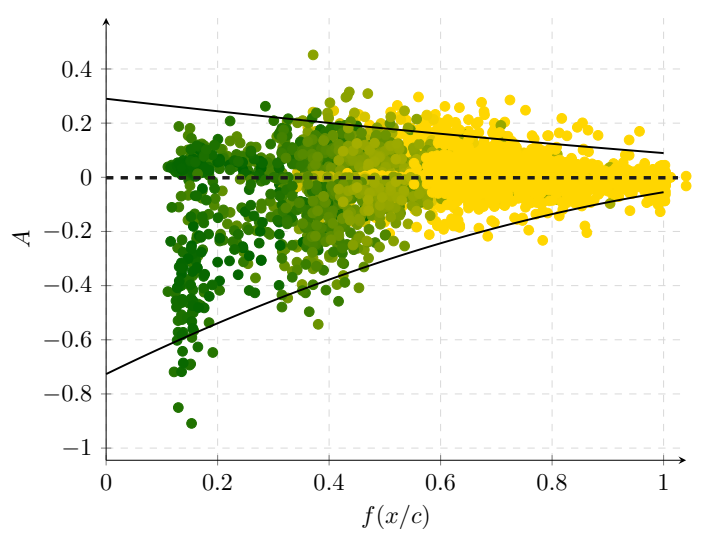

Figure 7.14: Shear layer maxima and minima on the $y$-axis plotted against the separation point $f(x / c)_{\omega=0}$ on the $x$-axis, with stall cycle following from attached flow (yellow) for full stall (green) at a pitch rate $\dot{\alpha}=17.14$ at $r / R=0.32$ with a least squares fit (black).

Results in figure 7.14 show that as separation approaches the leading edge, both the positive and negative amplitudes increase. The rate of increase for each displays an exponential growth, however the rate of growth in negative amplitude peaks is higher. The physical interpretation being that the external flow forms an upper boundary for the perturbation growth. As the external flow draws the low momentum fluid from within the stall region, the perturbation is able to penetrate the stalled volume, at times forming amplitudes which growth to $y / c \approx 70 \%$. The growth rate of the negative perturbations scales with the extent of separation, often on the order of the portion of the blade that is experiencing stall.

Figure 7.15 presents the spanwise trends for slow pitch rates in solid lines and fast pitch rates in dashed lines. The negative perturbations for planes between $r / R=0.19-0.32$ grow in magnitude as spanwise position move outboard, as well 


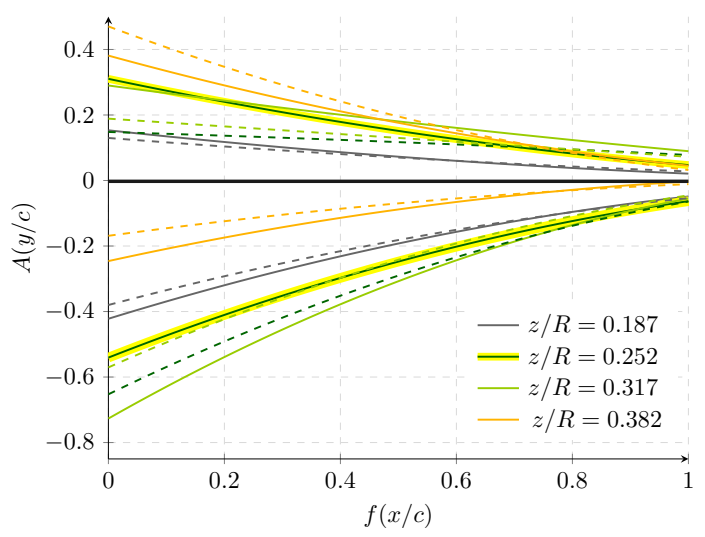

Figure 7.15: Least squares fit of shear layer amplitude maxima and minima plotted on the $y$-axis against the point of separation $f(x / c)_{\omega=0}$ on the $x$-axis for all planes $r / R=0.19-38$, and pitch rates $\dot{\alpha}=17.14$ (solid) and $\dot{\alpha}=34.28$ (dashed).

as showing increased growth rates moving along the span from the root to tip. Positive amplitude trends reveal that the disturbances are smallest at the inboard cross-section and largest in the middle two planes at $r / R=0.25$ and 0.32 . The ratio between maxima and minima within a single shear layer varies from 1 during attached flows to range from 0.5 at $r / R=0.19$ to 0.3 at $r / R=0.32$.

When exposed to a higher pitch rate, the positive amplitudes grow linearly as the separation approaches the leading edge, nearly equal across all planes. The negative amplitudes hold constant the inboard plane while there is an increase of $40 \%$ at $r / R=0.25$, and a slight decrease at $r / R=0.32$.

\subsection{External data set comparison}

An ancillary data set is analyzed by means of the methods/quantities applied in the previous sections. The data arises from flow over an oscillating OA209 airfoil 
supplied from measurements contained within the dynamic stall experiments by [82]. The airfoil modeled after a helicopter blade, the Reynolds number is set at $9.2 \times 10^{5}$. This value is significantly larger than the wind turbine data, as outlined in 3. The blade is pitched at a reduced frequency of $k=0.05$; well within the dynamic stall regime. The blade is continuously pitched through angles of attack from $12^{\circ} \leq \alpha \leq 28^{\circ}$ with a mean angle of $18^{\circ}$. The profile is significantly thinner than the profiles contained within a the root section of a wind turbine. As basis of comparison, it provides a geometric, Reynolds number and pitch rate that is significantly different.

The first comparison to be made is the separation point vorticity, which is plotted as a histogram. Figure 7.16 provides insight into the accumulation of vorticity at the point of separation for this experiment. The peak vorticity distribution is centered over $-70 \leq \omega^{*} \leq-60$. Additionally, the data set is negatively skewed, with excursions above the mean occurring more often. A similar result to the distributions found in the wind turbine cases which were tested using an $\dot{\alpha}=34.28$ pitch rate. This result supports the previous conclusion that when $\dot{\alpha}$ increases, the vorticity along the surface is able to build up beyond the mean separation point vorticity more frequently. The mean vorticity is also three times larger in magnitude due to the higher shear generated by a thinner blade profile and larger Reynolds number.

Next, the shear layer maximum and minimum perturbation amplitudes $A_{\max }$ and $A_{\min }$ are presented in figure 7.17. Employing the previously used scatter plot format, the perturbation amplitude is on the $y$-axis and the separation point on the $x$-axis. The separation point is defined by the vorticity zero within the stalled region. The color map, once again, demarkates the full stall regime in dark green 


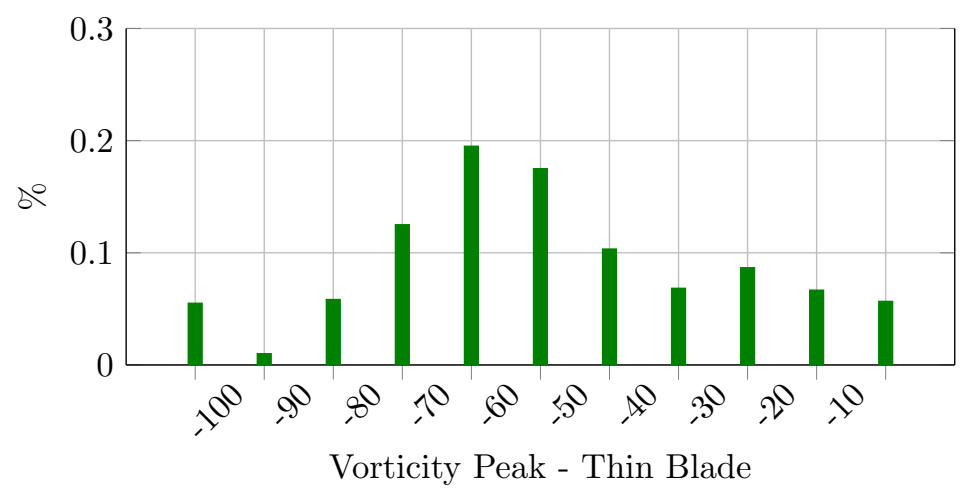

Figure 7.16: Separation point peak vorticity histogram for an oscillating OA209 airfoil with magnitude $\left(\omega^{*}\right)$ on the $x$-axis and frequency of occurrence (\%) on the $y$-axis.

and attached flow regime in yellow. First, looking at the plot itself, there is a slight bias to the perturbation towards negative values, but otherwise the two least squares fits are equal. The magnitude of the perturbations grows linearly as the separation point approaches the leading edge. During attached flow the amplitudes are on the order of $0.01 c$, while during full stall the perturbation amplitudes grow up to $0.1 \mathrm{~m}$ or $0.3 \mathrm{c}$. Comparing those amplitudes to the results presented in figure 7.15, it is seen that the amplitudes are considerably larger for the thicker aerodynamic profiles. Perturbations over the thicker profiles grow beyond $0.8 c$ in some cases. Additionally, the negative bias of the helicopter blade is less pronounced than that of the wind turbine, which is due to the significant curvature over the suction side of the thicker airfoils.

The shear layer origin, $S e p_{u}$, and the separation point, $S e p_{\omega}$, determined by the first occurrence of recirculation within the boundary layer are presented in figure 7.18. Here, the shear layer origin, $S e p_{u}$, is plotted on the $x$-axis and the zero vorticity separation point on the $y$-axis. The figure contains a targeted color scheme which 


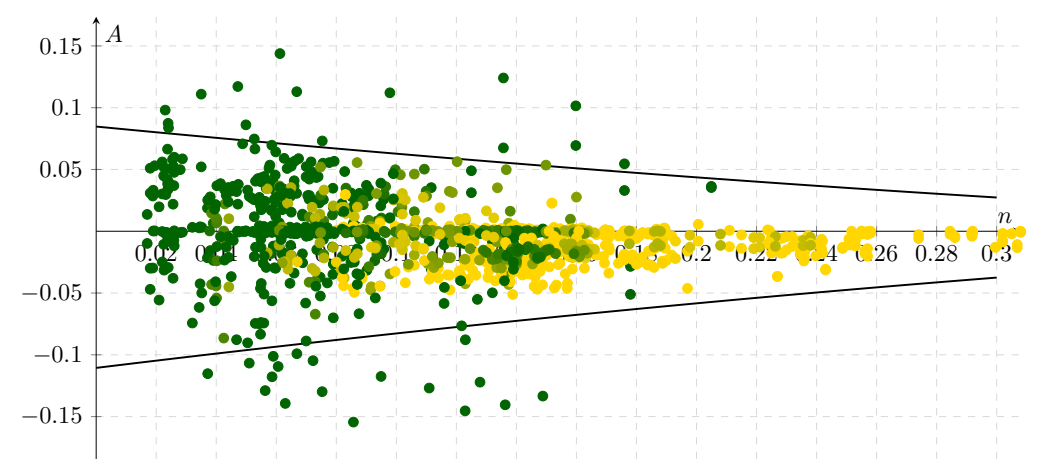

Figure 7.17: Shear layer maxima and minima on the $y$-axis plotted against the separation point $f(x / c)_{\omega=0}$ on the $x$-axis, with stall cycle following from attached flow (yellow) for full stall (green) at a pitch rate $\dot{\alpha}=17.14$ at $r / R=0.32$ with a least squares fit (black) for an oscillating OA209 airfoil.

captures attached flow in yellow and full stall in dark green. The results show that when flow is attached these two quantities are essentially equal. Both quantities extend along the black line which has a slope of $\partial f / \partial x=1$, with a small bias to the recirculation occurring farther downstream. During stall development, the variation between these two quantities grows reaching a maximum when the blade is in full stall. In some extreme cases, the difference between these two values can extend beyond $0.5 c$, which is attributed to the formation and shedding of surface vortices that remain near the blade surface as they are convected downstream. Recalling that the trend for the wind turbine blade, where the difference between the two quantities as the blade entered into full stall, was reduced during full stall. The difference between the helicopter blade and the wind turbine is attributed to the effect of the adverse pressure gradient, caused by surface curvature, which is significantly larger for thicker airfoils. Due to the relatively thin profile of the helicopter blade, the recirculation is able to extend further downstream during transitions due the 
relatively small adverse pressure gradient over the suction surface. That is, until the blade reaches its maximum angle of attack, in which case the two values return to being nearly equal when the separation point is pinned to the leading edge.

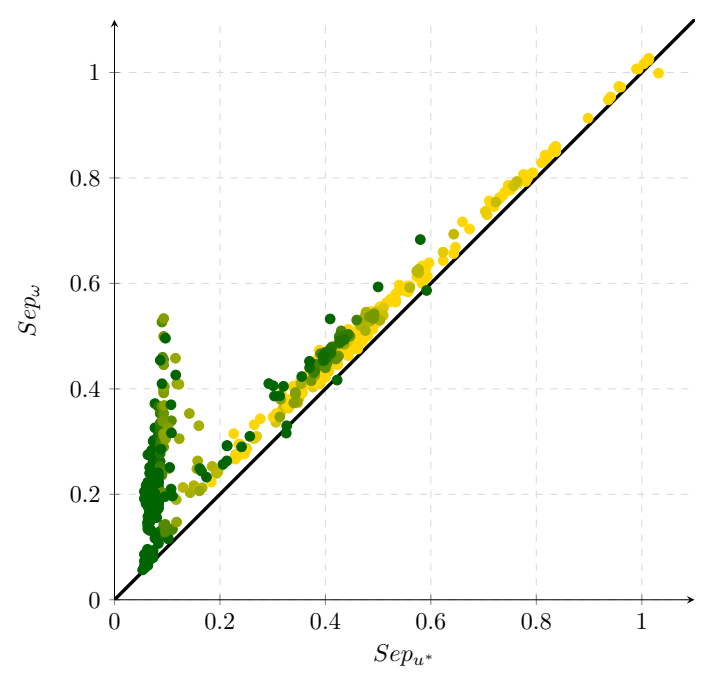

Figure 7.18: Shear layer origin based on the convective velocity of $0.48 U_{\infty}$ plotted against the fist occurrence of zero vorticity within the separated volume for an oscillating OA209 airfoil. tracking from attached flow stages (yellow) to full stall (green)

\subsection{Conclusions}

The expanded exploration of surface vorticity across the spanwise planes provides insight into the physical manifestation of separation on the inner root section of the experimental turbine blade. Consistently, for all planes and pitching regimes, the vorticity at the point of separation was a local peak, often in excess of $\omega^{*} \leq-15$. The variation in the peak surface vorticity was influenced by both the geometry of the aerodynamic profile, the pitch rate and the extent of stall that was present 
prior to the pitching cycle. For example, the inboard and outboard profiles are less sensitive to pitching rate than the middle two planes. In terms of separation progression, the inboard and outboard planes were less dramatic in the manner in which separation propagates as the angle of attack changes when compare to the middle two planes. The middle planes went through a stepwise transition, where the separation point would hesitate at mid-chord positions during which time the oscillation of the separation could move in excess of $\pm 40 \%$, in the extreme cases. Additionally, the surface vorticity reaches higher values overall in the middle two planes, further contributing to highly dynamic loading conditions during the transitional stages. The separation point vorticity also increases when the pitch rate increases for all planes. Despite those differences, the histograms also reveal that the separation point vorticity is remarkably consistent from plane to plane, speaking to the importance of this quantity as it applies to the unsteady separation over this airfoil. The role of Reynolds number, geometry and pitching rate are shown even more clearly when examining the same quantities over a much thinner aerodynamic profile which was pitched at an even faster rate and at a higher Reynolds number. In this case, the results show a much higher shear rate due to the geometry and Reynolds number lead to even greater $\left|\omega^{*}\right|$ peaks at the point of separation. Furthermore, the variation of the peaks throughout a stall cycle are further skewed by a higher pitching rate.

Looking deeper into the development and separation of the shear layer, the origin of the shear layer and the first point of zero vorticity are found to be dependent up the pitch rate, Reynold number and airfoil geometry. For faster pitching rates, the emergence of a persistent vorticity peak along the chord lead to large mid-span 
variations for both the wind turbine and helicopter blades push these locations apart during unsteady separation. The effect of a higher Reynolds number leads a contraction of these two quantities during attached flow. This differs significantly when compared to the wind turbine blade which experiences the opposite behavior at a lower Reynolds number. As for the effect of profile geometry, it is shown that the thicker profiles, which inherently have a more aggressive adverse pressure gradient over the suction surface experience more disparity between the shear layer origin and the emergence of recirculation. 


\section{Conclusions}

Dynamic stall cycle of an experimental three-dimensional wind turbine blade is investigated with time resolved PIV. Proper orthogonal decomposition is used to identify stall stages based on the contributions of the first four eigenmodes throughout a stall cycle. The first two POD modes were determined to capture the attached and separated flow states, respectively, and the third and fourth combined described stall development and reattachment. The values of the POD coefficients allowed the identification of the time scales for the five stall stages.

Applying an empirical mode decomposition to the stall stages reveals spatial scales which are directly related to the coherent structures formed within the shear layer. When the EMD modes are compared to the LCSs trends, the third EMD modes are found to describe flow structures that influence the LCS. With the information provided by these techniques, the formation and shedding of vortices is shown to influence the unsteady movement of the separation point. The connection between the movement of the separation point and the build up and shedding of surface vorticity motivated further investigation into the critical role of surface vorticity in unsteady aerodynamic transitions.

Upon evaluating the vorticity trend along the shear layer, a peak vorticity thresh- 
old was identified to persist throughout the stall cycle at the shear layer origin. Once recognized as a fundamental mechanism in the development of persistence of stall, the peak separation point vorticity statistics are evaluated and compared. The results reveal that separation point vorticity characteristics are fairly consistent between adjacent spanwise planes. The separation point vorticity is sensitive to the stall stage, inflow velocity, airfoil geometry, and the pitch rate. This finding is verified by comparing measurements from an external experiment, confirming that the separation point vorticity threshold is an quantifiable value which plays a critical role in unsteady separation.

The work documented in this dissertation provides significant insight into physical mechanisms which occur during unsteady separation. Results connect an airfoils geometry, Reynolds number, and pitching rate to many of the crucial fluid dynamic quantities that emerge during a stall cycle. The processes described in the current manuscript provide valuable details about the shear layer, separation point and coherent structure interaction during a stall cycle. These quantities are essential to a higher understanding of when and where stall will occur across the spectrum of aerodynamic bodies. With an understanding of how the growth of the separated shear layer, movement of the separation point, and the interaction of internal LCS are affected by the aerodynamic design, it becomes possible to greatly reduce the adverse affects of unsteady separation through advanced control mechanisms and enhanced modeling. The information provided will fundamentally improve the aerodynamic performance of any aerodynamic surface by providing engineers with a quantities of vibrational frequency and recirculation strength that can be avoided through passive or active controls. For example, if there is a known shedding frequency that 
occurs, the wing or body can be designed to have a higher natural frequency then the shedding frequency to prevent harmonic oscillation.

There is a opportunity to control separation by influencing the formation and growth of surface vorticity. Across spanwise planes and even in an additional experimental series, the occurrence of a peak vorticity at the separation point is consistently observed. While there are many factors that contribute to the magnitude of vorticity that leads to separation, the threshold magnitude can be identified for a specific application. Thus, modeling the separation criterion is made simpler by using that quantity to determine where separation occurs. Knowledge of where and when vorticity accumulates along the chord of an airfoil makes it possible to place passive vortex generators in strategic locations that prevent massive separation growth. By applying passive flow control, the peak vorticity may be held at a constant position rather than being free to move along the surface.

It has been shown that as the separation point in the mid-span progresses from the trailing edge to leading edge the separation point pauses temporarily at intermediate chord-wise nodes. If those nodes can be identified for the mid-span locations of an operational turbine, vortex generators could then be placed at those locations to reduce the fluctuating loads caused by shedding of vortices. To take this work further, application of such vorticity control would prove informative to improving the service life of wind turbine blades.

By quantifying the perturbation amplitudes within the shear layer, the fluctuation amplitude of the separation point is also obtainable. This quantity gives an amplitude of the loading dynamics throughout the stall cycle. Knowledge of the dynamic loading and unloading amplitudes informs the design which can lead to a 
reduction in fatigue breakdown caused by large movement of the separation point. By expanding this work to include fundamental systems such as a parametric study flow over a declining wedge would likely lead to scaling laws which capture the essence of unsteady separation. These laws could then be applied to more complex systems and lead to significant progress in many disciplines such as health care, transportation and energy production to name a few.

\subsection{Outlook}

For a more complete understanding of these quantities, it would be ideal to create a focused experimental series which alters single parameters such as Reynolds number, chordwise pressure gradient, and pitch rate. Doing so, would enable determination of the sensitivity of the separation vorticity to each parameter.

One such study that remains to be conducted would include measurements that are taken very close to the surface. In order to do so, a single aerodynamic profile would need to be studied in depth for a set of Reynolds numbers and pitching regimes. Once the peak vorticity and key separation points are identified, one can isolate specific locations along the surface for higher resolution measurements. High resolution surface measurements would provide direct physical insight into the phenomena that lead to separation. Through the application of surface pressure sensors in conjunction with high speed PIV at targeted chordwise locations, the relationship between the rotational velocity field and the surface pressure may finally be understood.

While the aforementioned study would provide deeper insight into two dimensional separation, there is still a need for developing three dimensional effects. Ex- 
tending the velocity measurements to volumetric PIV. Known as tomographic-PIV, this technique would captures three velocity components within the three spatial coordinates. The measurements during unsteady separation would show the spanwise depth of a stall cell as it propagates along the chord. These types of measurements would lead to three dimensional characterization of drag and lift performance of a given airfoil.

The final piece that could be added to the experimental design in order to capture the full complexity of a wind turbine is to include is rotation. If the model were rotating, the PIV measurements would need to be taken in a phased locked locations when the airfoil which is being pitched at a set rate passes through the field. If this study was extended such that each degree during a stall cycle is captured then the full unsteady separation physics would be measured. 


\section{Bibliography}

[1] I. Chapman, "The end of peak oil? why this topic is still relevant despite recent denials," Energy Policy, 2013.

[2] H. Waisman, J. Rozenberg, O. Sassi, and J.-C. Hourcade, "Peak oil profiles through the lens of a general equilibrium assessment," Energy Policy, vol. 48, no. 0 , pp. $744-753,2012$.

[3] R. Saidur, M. Islam, N. Rahim, and K. Solangi, "A review on global wind energy policy," Renewable and Sustainable Energy Reviews, vol. 14, no. 7, pp. $1744-$ 1762, 2010.

[4] WWEA, "The world wind energy report 2012," The world wind energy association, 2013.

[5] M. Islam, S. Mekhilef, and R. Saidur, "Progress and recent trends of wind energy technology," Renewable and Sustainable Energy Reviews, vol. 21, no. 0, pp. $456-468,2013$.

[6] T. Burton, N. Jenkins, D. Sharpe, and E. Bossanyi, Wind energy handbook. John Wiley \& Sons, 2011.

[7] H. Richard and M. Raffel, "Principle and applications of the background oriented schlieren (bos) method," Measurement Science and Technology, vol. 12, no. 9, p. 1576, 2001.

[8] J. Song, H. Luo, and T. L. Hedrick, "Three-dimensional flow and lift characteristics of a hovering ruby-throated hummingbird," Journal of The Royal Society Interface, vol. 11, no. 98, 2014.

[9] L. Prandtl, "Über flüssigkeitsbewegung bei sehr kleiner reibung," Int. Math. Kongr Heidelberg. Leipzig, 1904.

[10] S. Goldstein, "On laminar boundary-layer flow near a position of separation," The Quarterly Journal of Mechanics and Applied Mathematics, vol. 1, no. 1, pp. 43-69, 1948. 
[11] F. K. Moore, "On the separation of the unsteady laminar boundary layer," in Grenzschichtforschung/Boundary Layer Research, pp. 296-311, Springer, 1958.

[12] N. Rott, "Unsteady viscous flow in the vicinity of a stagnation point," Quarterly of Applied Mathematics, vol. 13, no. 4, pp. 444-451, 1956.

[13] W. R. Sears, "Some Recent Developments in Airfoil Theory," Journal of the Aeronautical Sciences, vol. 23, pp. 490-499, may 1956.

[14] L. L. van Dommelen and S.-F. Shen, "The spontaneous generation of the singularity in a separating laminar boundary layer," Journal of Computational Physics, vol. 38, no. 2, pp. 125-140, 1980.

[15] M. Tobak and D. J. Peake, "Topology of three-dimensional separated flows," Annual review of fluid mechanics, vol. 14, no. 1, pp. 61-85, 1982.

[16] S. J. Chapman, "A mean-field model of superconducting vortices in three dimensions," SIAM Journal on Applied Mathematics, vol. 55, no. 5, pp. 1259$1274,1995$.

[17] J. E. Simpson, "Gravity currents in the laboratory, atmosphere, and ocean," Annual Review of Fluid Mechanics, vol. 14, no. 1, pp. 213-234, 1982.

[18] A. Surana, G. B. Jacobs, O. Grunberg, and G. Haller, "An exact theory of three-dimensional fixed separation in unsteady flows," Physics of Fluids, vol. 20, no. 10, p. 107101, 2008.

[19] S.-F. Shen, "Unsteady separation according to the boundary-layer equation," Advances in Applied Mechanics, vol. 18, pp. 177-220, 1979.

[20] L. Van Dommelen and S. Shen, "The genesis of separation," in Numerical and physical aspects of aerodynamic flows, pp. 293-311, Springer, 1982.

[21] L. L. Van Dommelen and S. J. Cowley, "On the lagrangian description of unsteady boundary-layer separation. part 1. general theory," Journal of Fluid Mechanics, vol. 210, pp. 593-626, 1990.

[22] R. Adrian, C. Meinhart, and C. Tomkins, "Vortex organization in the outer region of the turbulent boundary layer," Journal of Fluid Mechanics, vol. 422, pp. 1-54, 2000.

[23] K. Cassel and A. Conlisk, "Unsteady separation in vortex-induced boundary layers," Philosophical Transactions of the Royal Society of London A: Mathematical, Physical and Engineering Sciences, vol. 372, no. 2020, p. 20130348, 2014. 
[24] A. Prasad and C. H. K. Williamson, "The instability of the shear layer separating from a bluff body," Journal of Fluid Mechanics, vol. 333, pp. 375-402, Feb. 1997.

[25] D. Tritton, Physical Fluid Dynamics. Oxford Science Publ, Clarendon Press, 1988.

[26] S. K. Roberts and M. I. Yaras, "Large-eddy simulation of transition in a separation bubble," Journal of fluids engineering, vol. 128, no. 2, pp. 232-238, 2006.

[27] J. Wu, R. Tramel, F. Zhu, and X. Yin, "A vorticity dynamics theory of threedimensional flow separation," Physics of Fluids, vol. 12, no. 8, pp. 1932-1954, 2000 .

[28] V. V. Sychev, Asymptotic theory of separated flows. Cambridge University Press, 1998.

[29] R. J. Barthelmie, S. T. Frandsen, M. N. Nielsen, S. C. Pryor, P.-E. Rethore, and H. E. Jø rgensen, "Modelling and measurements of power losses and turbulence intensity in wind turbine wakes at Middelgrunden offshore wind farm," Wind Energy, vol. 10, pp. 517-528, Nov. 2007.

[30] E. Anahua, S. Barth, and J. Peinke, "Markovian power curves for wind turbines," Wind Energy, vol. 11, pp. 219-232, May 2008.

[31] M. S. Melius, M. Tutkun, and R. B. Cal, "Solution of the Fokker-Planck equation in a wind turbine array boundary layer," Physica D: Nonlinear Phenomena, vol. 280-281, pp. 14-21, July 2014.

[32] N. Hamilton, H. Suk Kang, C. Meneveau, and R. B. Cal, "Statistical analysis of kinetic energy entrainment in a model wind turbine array boundary layer," Journal of Renewable and Sustainable Energy, vol. 4, no. 6, p. 063105, 2012.

[33] L. P. Chamorro and F. Porté-Agel, "A Wind-Tunnel Investigation of WindTurbine Wakes: Boundary-Layer Turbulence Effects," Boundary-Layer Meteorology, vol. 132, pp. 129-149, Apr. 2009.

[34] Z. Du and M. Selig, "A 3-D stall-delay model for horizontal axis wind turbine performance prediction," AIAA Paper, vol. 21, 1998.

[35] R. Wilson, "Wind-turbine aerodynamics," Journal of Wind Engineering and Industrial Aerodynamics, vol. 5, no. 3, pp. 357-372, 1980. 
[36] H. Glauert, Airplane Propellers. Springer Berlin Heidelberg, 1935.

[37] D. Hu, O. Hua, and Z. Du, "A study on stall-delay for horizontal axis wind turbine," Renewable Energy, vol. 31, pp. 821-836, May 2006.

[38] P. J. Schubel and R. J. Crossley, "Wind Turbine Blade Design," Energies, vol. 5, pp. 3425-3449, Sept. 2012.

[39] A. C. Hansen and C. P. Butterfield, "Aerodynamics of horizontal-axis wind turbines," Annual Review of Fluid Mechanics, vol. 25, no. 1, pp. 115-149, 1993.

[40] W. Timmer and R. van Rooy, "Thick airfoils for horizontal axis wind turbines," Journal of Wind Engineering and Industrial Aerodynamics, vol. 39, no. 1-3, pp. $151-160,1992$.

[41] H. Himmelskamp, Profile Investigations on a Rotating Airscrew. Reports and translations / MAP Völenrode, MAP, 1947.

[42] H. Dwyer and W. McCroskey, "Crossflow and unsteady boundary-layer effects on rotating blades," AIAA Journal, vol. 9, no. 8, pp. 1498-1505, 1971.

[43] L. Vermeer, J. Sørensen, and A. Crespo, "Wind turbine wake aerodynamics," Progress in aerospace sciences, vol. 39, pp. 467-510, 2003.

[44] Z. Du and M. Selig, "The effect of rotation on the boundary layer of a wind turbine blade," Renewable Energy, vol. 20, pp. 167-181, June 2000.

[45] N. R. Garcia, Unsteady viscous-inviscid interaction technique for wind turbine airfoils. PhD thesis, Ph. D. thesis, Technical university of Denmark, 2011.

[46] J. Sørensen, P. Larsen, B. Pedersen, and J. Jensen, Three-Level, ViscousInviscid Interaction Technique for the Prediction of Separated Flow Past Rotating Wing. PhD thesis, 1986.

[47] C. Bak, "Sensitivity of key parameters in aerodynamic wind turbine rotor design on power and energy performance," Journal of Physics: Conference Series, vol. 75, p. 012008, July 2007.

[48] C. Lindenburg, "Investigation into rotor blade aerodynamics," ECN Report: ECN-C-03-025, 2003.

[49] K. Mulleners and M. Raffel, "Dynamic stall development," Experiments in fluids, vol. 54, no. 2, pp. 1-9, 2013. 
[50] N. Sørensen, J. Sørensen, S. Voutsinas, H. Madsen, and M. Hansen, State of the art in wind turbine aerodynamics and aeroelasticity, vol. 42. 2006.

[51] G. Haller, "Exact theory of unsteady separation for two-dimensional flows," Journal of Fluid Mechanics, vol. 512, pp. 257-311, July 2004.

[52] W. C. Reynolds and L. Carr, Review of unsteady, driven, separated flows. 1985.

[53] C. Shih, L. Lourenco, L. Van Dommelen, and A. Krothapalli, "Unsteady flow past an airfoil pitching at a constant rate," AIAA journal, vol. 30, no. 5, pp. 1153-1161, 1992.

[54] Y. Li, K.-J. Paik, T. Xing, and P. M. Carrica, "Dynamic overset cfd simulations of wind turbine aerodynamics," Renewable Energy, vol. 37, no. 1, pp. 285-298, 2012 .

[55] B. F. Xu, Y. Yuan, and T. G. Wang, "Development and application of a dynamic stall model for rotating wind turbine blades," Journal of Physics: Conference Series, vol. 524, no. 1, p. 012133, 2014.

[56] J. Jeong and F. Hussain, "On the identification of a vortex," Journal of Fluid Mechanics, vol. 285, pp. 69-94, 1995.

[57] K. Mulleners and M. Raffel, "The onset of dynamic stall revisited," Experiments in Fluids, vol. 52, no. 3, pp. 779-793, 2012.

[58] L. Graftieaux, M. Michard, and N. Grosjean, "Combining PIV, POD and vortex identification algorithms for the study of unsteady turbulent swirling flows," Measurement Science \&3 Technology, vol. 12, pp. 1422-1429, 2001.

[59] L. Sirovich and M. Kirby, "Low-dimensional procedure for the characterization of human faces," J. Opt. Soc. Am. A, vol. 4, pp. 519-524, Mar 1987.

[60] P. Cizmas, a. Palacios, T. O'Brien, and M. Syamlal, "Proper-orthogonal decomposition of spatio-temporal patterns in fluidized beds," Chemical Engineering Science, vol. 58, pp. 4417-4427, Oct. 2003.

[61] K. Saranyasoontorn and L. Manuel, "Low-dimensional representations of inflow turbulence and wind turbine response using proper orthogonal decomposition," Journal of Solar Energy Engineering, vol. 127, pp. 553-562, 072005.

[62] J. Taylor and M. Glauser, "Towards practical flow sensing and control via pod and lse based low-dimensional tools," Journal of Fluids Engineering, vol. 126, no. 3, pp. 337-345, 2004. 
[63] G. Kerschen, J.-c. Golinval, A. F. Vakakis, and L. a. Bergman, "The Method of Proper Orthogonal Decomposition for Dynamical Characterization and Order Reduction of Mechanical Systems: An Overview," Nonlinear Dynamics, vol. 41, pp. 147-169, Aug. 2005.

[64] N. E. Huang, Z. Shen, S. R. Long, M. C. Wu, H. H. Shih, Q. Zheng, N.-C. Yen, C. C. Tung, and H. H. Liu, "The empirical mode decomposition and the hilbert spectrum for nonlinear and non-stationary time series analysis," in Proceedings of the Royal Society of London A: Mathematical, Physical and Engineering Sciences, vol. 454, pp. 903-995, The Royal Society, 1998.

[65] N. E. Huang, M.-L. C. Wu, S. R. Long, S. S. Shen, W. Qu, P. Gloersen, and K. L. Fan, "A confidence limit for the empirical mode decomposition and hilbert spectral analysis," in Proceedings of the Royal Society of London A: Mathematical, Physical and Engineering Sciences, vol. 459, pp. 2317-2345, The Royal Society, 2003.

[66] Z. Wu and N. E. Huang, "Ensemble empirical mode decomposition: a noiseassisted data analysis method," Advances in adaptive data analysis, vol. 1, no. 01, pp. 1-41, 2009.

[67] M. E. Torres, M. A. Colominas, G. Schlotthauer, and P. Flandrin, "A complete ensemble empirical mode decomposition with adaptive noise," in Acoustics, speech and signal processing (ICASSP), 2011 IEEE international conference on, pp. 4144-4147, IEEE, 2011.

[68] Z. Wu, N. E. Huang, and X. Chen, "The multi-dimensional ensemble empirical mode decomposition method," Advances in Adaptive Data Analysis, vol. 1, no. 03, pp. 339-372, 2009.

[69] J. C. Nunes, Y. Bouaoune, E. Delechelle, O. Niang, and P. Bunel, "Image analysis by bidimensional empirical mode decomposition," Image and vision computing, vol. 21, no. 12, pp. 1019-1026, 2003.

[70] J. C. Nunes, S. Guyot, and E. Deléchelle, "Texture analysis based on local analysis of the bidimensional empirical mode decomposition," Machine Vision and applications, vol. 16, no. 3, pp. 177-188, 2005.

[71] M. Farazmand and G. Haller, "Computing lagrangian coherent structures from their variational theory," Chaos: An Interdisciplinary Journal of Nonlinear Science, vol. 22, no. 1, p. 013128, 2012. 
[72] S. C. Shadden, F. Lekien, and J. E. Marsden, "Definition and properties of lagrangian coherent structures from finite-time lyapunov exponents in twodimensional aperiodic flows," Physica D: Nonlinear Phenomena, vol. 212, no. 3, pp. 271-304, 2005.

[73] G. Haller, "A variational theory of hyperbolic lagrangian coherent structures," Physica D: Nonlinear Phenomena, vol. 240, no. 7, pp. 574-598, 2011.

[74] M. Raffel, C. E. Willert, J. Kompenhans, et al., Particle image velocimetry: a practical guide. Springer, 2013.

[75] K. Gersten and H. Schlichting, Boundary-Layer Theory. Berlin, Heidelberg: Springer Berlin Heidelberg, 2009.

[76] M. Melius, R. B. Cal, and K. Mulleners, "Dynamic stall of an experimental wind turbine blade," Physics of Fluids, vol. 28, no. 3, 2016.

[77] Z. Wilson, M. Tutkun, and R. Cal, "Identification of lagrangian coherent structures in a turbulent boundary layer," Journal of Fluid Mechanics, vol. 728, pp. 396-416, 2013.

[78] F. Wang and X. Li, "The stagnant thermal conductivity of porous media predicted by the random walk theory," International Journal of Heat and Mass Transfer, vol. 107, pp. 520-533, 2017.

[79] A. Veluchamy, L. Ballerini, V. Vitart, C. Hayward, K. Schraut, P. Joshi, H. Campbell, M. Kirin, D. Relan, S. Harris, et al., "Novel locus influencing retinal venular tortuosity is also associated with risk of coronary artery disease," bioRxiv, p. 121012, 2017.

[80] S. Yarusevych, P. E. Sullivan, and J. G. Kawall, "On vortex shedding from an airfoil in low-reynolds-number flows," Journal of Fluid Mechanics, vol. 632, pp. 245-271, 2009.

[81] H. Y. Toda and T. Yamamoto, "Statistical inference in vector autoregressions with possibly integrated processes," Journal of econometrics, vol. 66, no. 1, pp. 225-250, 1995.

[82] K. Mulleners and M. Raffel, "The onset of dynamic stall revisited," Experiments in fluids, vol. 52, no. 3, pp. 779-793, 2012. 